\title{
Prevention of twin pregnancies in IVF by single embryo transfer
}

Citation for published version (APA):

van Montfoort, A. P. (2007). Prevention of twin pregnancies in IVF by single embryo transfer. [Doctoral Thesis, Maastricht University]. Datawyse / Universitaire Pers Maastricht.

https://doi.org/10.26481/dis.20070613am

Document status and date:

Published: 01/01/2007

DOI:

10.26481/dis.20070613am

Document Version:

Publisher's PDF, also known as Version of record

\section{Please check the document version of this publication:}

- A submitted manuscript is the version of the article upon submission and before peer-review. There can be important differences between the submitted version and the official published version of record.

People interested in the research are advised to contact the author for the final version of the publication, or visit the DOI to the publisher's website.

- The final author version and the galley proof are versions of the publication after peer review.

- The final published version features the final layout of the paper including the volume, issue and page numbers.

Link to publication

\footnotetext{
General rights rights.

- You may freely distribute the URL identifying the publication in the public portal. please follow below link for the End User Agreement:

www.umlib.nl/taverne-license

Take down policy

If you believe that this document breaches copyright please contact us at:

repository@maastrichtuniversity.nl

providing details and we will investigate your claim.
}

Copyright and moral rights for the publications made accessible in the public portal are retained by the authors and/or other copyright owners and it is a condition of accessing publications that users recognise and abide by the legal requirements associated with these

- Users may download and print one copy of any publication from the public portal for the purpose of private study or research.

- You may not further distribute the material or use it for any profit-making activity or commercial gain

If the publication is distributed under the terms of Article $25 \mathrm{fa}$ of the Dutch Copyright Act, indicated by the "Taverne" license above, 


\section{Prevention of twin pregnancies in IVF by single embryo transfer}

Aafke P.A. van Montfoort 
Copyright (c) 2007 Aafke P.A. van Montfoort, Maastricht ISBN: 978-90-5278-633-9

Coverdesign and lay-out: Ruud Thijssen, Eindhoven Printed by Datawyse, Universitaire Pers Maastricht 


\title{
Prevention of twin pregnancies in IVF by single embryo transfer
}

\author{
Proefschrift \\ ter verkrijging van de graad van doctor \\ aan de Universiteit Maastricht, \\ op gezag van de Rector Magnificus, \\ Prof. Mr. G.P.M.F. Mols, \\ volgens het besluit van het College van Decanen, \\ in het openbaar te verdedigen \\ op woensdag 13 juni om 12.00 uur door
}

Aafke Philomena Anna van Montfoort geboren te Weert op 2 oktober 1979

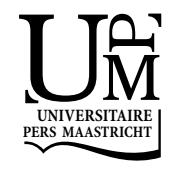




\section{Promotores}

Prof. dr. J.L.H. Evers

Prof. dr. J.P.M. Geraedts

\section{Copromotor}

Dr. J.C.M. Dumoulin

Beoordelingscommissie

Prof. dr. J. de Haan (voorzitter)

Prof. dr. B.C.J.M. Fauser (Universitair medisch centrum Utrecht)

Prof. dr. J. Gerris (Universitair ziekenhuis Gent, België)

Prof. dr. A.W. Griffioen

Prof. dr. M.H. Prins

The studies presented in this thesis were financially supported by a research grant (number 945-12-014) from the Dutch Organisation for Health Research and Development (ZonMW) and the Dutch Health Insurance Board (CVZ) in a joint research programme on health technology assessment of infertility.

Publication of this thesis was financially supported by:

Organon Nederland BV 
Aan Pap en Mam Aan Bregje 
Abbreviations

p. 9

Chapter 1 General Introduction

p. 11

Chapter 2 Elective single embryo transfer (eSET) policy

p. 27 in the first three IVF/ICSI treatment cycles

Chapter 3 In unselected patients, elective single embryo

p. 37 transfer prevents all multiples, but results in significanlty lower pregnancy rates compared with double embryo transfer: a randomized controlled trial

Chapter 4 Single versus double embryo transfer: cost-

p. 51 effectiveness analysis alongside a randomized clinical trial

Chapter 5 ESET irrespective of the availability of a good

p. 73 quality embryo in the first cycle only is not effective in reducing overall twin pregnancy rates

Chapter 6 Early cleavage is a valuable addition to existing

p. 87 embryo selection parameters: a study using single embryo transfers

Chapter $7 \quad$ Differential gene expression in cumulus cells as

p. 99 a prognostic indicator of embryo viability: a microarray analysis

Chapter 8 General discussion

p. 121

References

p. 135

Summary

p. 149

Samenvatting

p. 152

Dankwoord

p. 155

Curriculum Vitae and publications

p. 158 


\section{Abbreviations}

ART Artificial reproductive technology

AUCROC Area under receiver operating curve

CCND2 Cyclin D2

$\mathrm{CP}$ Cerebral palsy

CSET Compulsory single embryo transfer

(SET when only one embryo is available for transfer)

CTNND1 Catenin delta-1

CXCR4 CXC chemokine receptor 4

DET Double embryo transfer

DHCR7 7-dehydrocholesterol reductase

DVL3 Dishevelled dsh homolog 3

DZ Dizygotic

EC Early cleavage

ESET Elective single embryo transfer

ET Embryo transfer

FISH Fluorescent in situ hybridization

GP General practitioner

GPX3 Glutathione peroxidase 3

GQE Good quality embryo

hCG Human chorionic gonadotropin

HSPB 1 Heatshock 27 kDa protein 1

ICER Incremental cost-effectiveness ratio

ICSI Intracytoplasmic sperm injection

IUI Intra-uterine insemination

IVF In vitro fertilization

MNB Multinucleated blastomere

$\mathrm{MZ}$ Monozygotic

NEC Non-early cleavage

NICU Neonatal intensive care unit

NPB Nucleolar precursor body

OHSS Ovarian hyperstimulation syndrome

OPU Ovum pick up

OR Odds ratio

PGD Preimplantation genetic diagnosis

PGS-AS Preimplantation genetic screening - aneuploidy screening

PN Pronucleus

PR Pregnancy rate

QRT-PCR Quantitative real-time polymerase chain reaction

RCT Randomized controlled trial

ROS Reactive oxygen species

SET Single embryo transfer

SP Singleton pregnancy

TLDA Taqman low density array

TP Twin pregnancy

TRIM28 Tripartite motif-containing 28 
CHAPTER 1

General Introduction 


\section{Preface}

Human reproduction can be considered very inefficient. Per menstrual cycle, around $30 \%$ of the conceptions is lost before implantation, another $30 \%$ is lost after implantation but before recognition of a pregnancy by the woman and $10 \%$ is lost after clinical confirmation of a pregnancy. Eventually only $30 \%$ of all conceptions will result in a live born child (Macklon et al., 2002). Within one year, around $90 \%$ of the couples trying to achieve a pregnancy, will succeed (Taylor, 2003). However, about 10\% of the couples is confronted with subfertility, i.e. not achieving a pregnancy in spite of regular intercourse aimed at conception for more than 12 months (Beurskens et al., 1995; Evers, 2002).

The aetiology is diverse: ovulation failure (25\%), semen anomalies (30\%), tubal factors (20\%), endometriosis (5\%) and unexplained (25\%). This exceeds $100 \%$ as some couples have more than one cause of subfertility. Roughly, it can be said that in $30 \%$ of the couples subfertility is caused by a female factor, in $30 \%$ by a male factor and in another $30 \%$ by a combination of both a male and a female factor. For the remaining $10 \%$ the cause is unknown (Cahill and Wardle, 2002).

Depending on the diagnosis, an appropriate treatment like ovulation induction, intrauterine insemination (IUI) or in vitro fertilization (IVF) with or without intracytoplasmic sperm injection (ICSI) can be applied. The latter is the last resort for severe subfertility cases and longstanding unexplained subfertility. More than 257,000 IVF cycles are performed each year in Europe, of which almost 15,000 in The Netherlands. Nowadays, depending on the country, 1.3-4.2\% of the newborns in Europe are born after the application of assisted reproductive technologies (ART) (Nyboe Andersen et al., 2006).

\section{Risks and complications of IVF and ICSI}

Although effort is made with IVF and ICSI to mimic the natural situation as closely as possible, there are stages that are different in comparison to the natural cycle. These differences entail a number of potential risks for the patient as well as for the embryo and/or the children to be born.

Ovarian stimulation: The administration of gonadotrophins for follicle development results in approximately $0.2-1 \%$ of the patients in severe ovarian hyperstimulation syndrome (OHSS). This can, due to the accompanying electrolyte disorders and thrombo-embolic accidents, even lead to death of the patient (Abramov et al., 1999; Delvigne and Rozenberg, 2003). 
Fertilization and in vitro culture: The invasive ICSI technique and the accompanying injection of biochemical components together with the spermatozoon, might be the cause of the increase in aneuploidy and chromosomal anomalies reported in ICSI children (Liebaers et al., 1995; Loft et al., 1999; Bonduelle et al., 2002). It is however more likely that they are caused by the higher aneuploidy rate in the sperm cells of subfertile men (Bonduelle et al., 2002).

Also the embryo culture is reason for concern. Components of the culture medium can disrupt the imprinted expression of genes in mice (Khosla et al., 2001). The prevalence of imprinting disorders in human is $2.9 \%-4.2 \%$ after ART (Gosden et al., 2003; Sutcliffe et al., 2006), although the study population in both studies is very small ( $n=214$ and 213 respectively).

Multiple embryo transfer: To increase the success rate for IVF, ovarian stimulation was introduced to generate multiple embryos (Speirs et al., 1983; Gronow et al., 1985). The transfer of multiple embryos resulted in an increase in the multiple pregnancy rate (multiple PR), as described in paragraph 1.3. In Europe in 2002, an average of 2.2 embryos was transferred per cycle, resulting in $75.5 \%$ singleton, $23.2 \%$ twin and $1.3 \%$ triplet pregnancies (Nyboe Andersen et al., 2006). In the USA, an average of 2.8 embryos was transferred in 2002, resulting in $64.8 \%$ singleton, $31.7 \%$ twin and 3.5\% triplet or higher order pregnancies (Wright et al., 2005). This means that around $40 \%$ of the children born after ART in Europe and 55\% in the USA originated from a multiple pregnancy, compared to $2-3 \%$ in the general population (Bergh et al., 1999). Regarding the incidence and the associated complications as described in paragraph 1.4, a multiple pregnancy is regarded as one of the most serious complications of IVF treatment. This thesis is focussed on the reduction of the multiple and especially the twin PR.

\section{Aetiology and prevalence of twin pregnancies}

Twin gestations can be divided into monozygotic and dizygotic, depending on the number of oocytes that have been fertilized. An embryo resulting from one fertilized oocyte can split, resulting in a monozygotic (MZ) twin, whereas the fertilization of two oocytes results in a dizygotic (DZ) twin (ESHRE Capri Workshop, 2000).

As $M Z$ twinning is a random embryological event, largely independent of environmental factors, the rate remained constant throughout the world at 4 per 1000 live births (ESHRE Campus Course, 2001). In ART live births this rate is doubled (Schachter et al., 2001). MZ twinning is positively correlated with ovarian stimulation (Derom et al., 1991; Schachter et al., 2001), 
blastocyst transfer (Milki et al., 2003; Jain et al., 2004) and zona pellucida micromanipulation (Slotnick and Ortega, 1996; Abusheikha et al., 2000).

Spontaneous DZ twinning (with an incidence of 1.2\% (ESHRE Capri Workshop, 2000)) is affected by more factors like maternal age, parity, ethnic background, nutrition and heredity (Bortolus et al., 1999). Its incidence increases four-fold from the maternal age of 15 to 37 years and two-fold from parities of 0 to 10 (Tong and Short, 1998). Also ethnicity is involved as the highest rates are reported in Nigeria (50/1000 live births) (ESHRE Capri Workshop, 2000) and the lowest in Japan (4/1000 live births) (Wenstrom and Gall, 1988). In general, the DZ twin rate is about 8/1000 in whites, about twice as large in the black community and less than half as large in Asians (Bortolus et al., 1999). Explanations for this ethnic difference might be differences in basal FSH concentration (Nylander, 1981), genetic predisposition or malnutrition (Bortolus et al., 1999).

Before the onset of ART, the multiple PR was gradually declining in several western countries (Botting et al., 1987; Tuppin et al., 1993; Luke, 1994). This reduction was attributable to a decrease in the incidence of risk factors like enhanced maternal age and multiparity (Senat et al., 1998). However, in the mid-1970s the multiple PR started to increase again. This was largely due to the introduction of subfertility treatment (Tuppin et al., 1993; Dunn and Macfarlane, 1996). The first series of IVF treatments, performed in the late seventies, was attempted using the single oocyte from the natural cycle. The PR was very low $(4 / 32=13 \%)$, as only three children were delivered of which one died after a premature birth (Edwards, 1996). Soon it was realised that the success rate per cycle could be increased by transferring multiple embryos, instead of one (Speirs et al., 1983; Gronow et al., 1985). To achieve these multiple embryos, ovarian stimulation was introduced as a part of the IVF treatment (Edwards, 1996). Fifteen to twenty years after this introduction the twin PR was increased 1.3-1.7 fold and the higher order multiple PR 2-4 fold in several European countries and the USA (Van Duivenboden et al., 1991; Tuppin et al., 1993; Dunn and Macfarlane, 1996; Westergaard et al., 1997; Bergh et al., 1999; Martin and Park, 1999). These increases were not caused by multiple embryo transfer in IVF alone. Also other types of subfertility treatment, like ovulation induction in anovulatory women or superovulation in combination with intrauterine insemination (IUI) were involved as well as an increase in maternal age at childbirth. Bergh et al. (1999) demonstrated that the advanced age of childbearing women was responsible for one third of the increase in twin pregnancies. Another third is due to an increase in the use of IVF and the remaining third because of an increase in ovarian stimulation. This is in accordance with the findings of a study of 169 multiple pregnancies in UK, showing that $34 \%$ resulted from ovarian stimulation without IVF and 35\% with IVF (Doyle, 1996). 


\section{Obstetric risks, neonatal complications and costs of twin pregnancies}

\section{Maternal complications}

Table I summarizes data regarding the incidence and risk of maternal complications in singleton and twin pregnancies. Besides a small but significantly increased risk of maternal mortality (Senat et al., 1998), women carrying a twin are at an increased risk of several disorders and complications like hypertension, hemorrhage, anemia, and delivery by Caesarean section (Spellacy et al., 1990; Stones et al., 1993; Santema et al., 1995; Senat et al., 1998; Sibai et al., 2000; Sebire et al., 2001). Hypertension is the major maternal complication in twin pregnancies (Santema et al., 1995: Senat et al., 1998). A distinction has to be made between nonproteinuric and proteinuric hypertension (pre-eclampsia) (Senat et al., 1998; Norwitz et al., 2005). The latter is accompanied by higher maternal and fetal morbidity and mortality as compared to the first (Senat et al., 1998; Norwitz et al., 2005). Twin pregnancies also have an increased risk of antepartum hemorrhage (partly caused by abruptio placentae) (Spellacy et al., 1990) and postpartum hemorrhage (Stones et al., 1993; Sebire et al., 2001 ). This is also visualised by the increased incidence of transfusions ( $8 \%$ for twin and $2.5 \%$ for singleton deliveries) (Kinzler et al., 2000) and anemia ( $9.4 \%$ vs $4.1 \%$, respectively) (Spellacy et al., 1990). Finally an almost threefold risk of Caesarean section, which has an increased risk of maternal morbidity and mortality, has been reported (Sebire et al., 2001).

Neonatal outcome

The most frequently occurring complications in twin pregnancies are preterm delivery and low birthweight (Table II). The mean birthweight of a twin is $2300 \mathrm{~g}$ against $3300 \mathrm{~g}$ for a singleton (Spellacy et al., 1990; Doyle, 1996; Kinzler et al., 2000). The mean gestational age of a twin pregnancy is 35.6 weeks, while a singleton pregnancy is delivered on average at 39.0 weeks (Kinzler et al., 2000). The aetiology of preterm delivery in twin births, in a study analyzing 432 twin pregnancies, was spontaneous (54\%), premature rupture of membranes (22\%) and induced deliveries due to maternal hypertension, fetal growth restriction or fetal death $(23 \%)$ (Gardner et al., 1995). Preterm delivery and low birthweight are the major causes of neonatal morbidity and mortality.

Both the early fetal death rate and the still birth and neonatal mortality rate are increased in twin pregnancies (Table II). Early fetal demise presenting as vanishing sacs is identified especially after ART pregnancies, as these pregnancies are followed-up more frequently during early pregnancy as compared to spontaneous pregnancies. Of the pregnancies with two fetal sacs, approximately $30 \%$ will result in one fetal sac and $<10 \%$ in no fetal sac (Landy and Keith, 1998). For spontaneous pregnancies, De Neubourg et al. (2004) reported a first trimester pregnancy loss of $15.4 \%$ after IVF. 
Besides the increased mortality rate, also the increased morbidity is attributable to the low birth weight and preterm delivery. Among other complications, a 6.6-fold risk of respiratory distress syndrome and a 4.5-fold increase in intraventricular hemorrhage was observed in twins compared to singletons (Gardner et al., 1995). This increase in neonatal complications results in an increased admittance to neonatal intensive care units (NICU) of twin children (20.6\% of twin and $3.9 \%$ singleton) (Sebire et al., 2001 ).

Twins also have an increase in long-term medical and developmental problems like chronic illness, communicative disorders and neurological impairments (Luke and Keith, 1992), with cerebral palsy (CP) being the most important (Petterson et al., 1993). CP is characterized by movement disabilities attributed to disturbances in the developing fetal or neonatal brain. However, Pinborg et al. (2003) did not found a difference between IVF/ICSI twin or singletons regarding severe neurological disabilities. Due to shared parental attention the language development can be adversely affected (Mittler, 1976; Pinborg et al., 2003) and the death of one of the twins can lay a psychological burden on the other twin (Bryan and Read, 1995). Furthermore, twins are a predictor for more marital stress (more crises in the relationship and thoughts about divorce) as well as for less marital benefit (Pinborg et al., 2003).

\section{Economic implications}

Due to these maternal and neonatal complications, the healthcare costs are high in multiple pregnancies. It has been determined that these costs up to six weeks after delivery are $€ 13,469$ per twin compared to $€ 2,549$ per singleton pregnancy (Lukassen et al., 2004). This difference is mainly caused by the maternal hospitalization, resulting from the increased incidence of preterm delivery, and the associated admittance of the neonates to the $\mathrm{NICU}$ together with diagnostic procedures and drug therapies. For a minor part, the difference could be attributed to extra examinations during the antenatal period. The difference in costs will increase even more when the lifetime costs for handicaps, long-term chronic complications and developmental disabilities are included.

For instance for the hospital care utilization of IVF/ICSI twins vs singletons an odds ratio (OR) of 2.44 was determined. When the premature infants were excluded, this OR was still 1.4 (Pinborg et al., 2004). Wolner-Hanssen calculated that the average costs for care of severely handicapped children was almost 14 times higher for twin pregnancies ( $€ 20477)$ compared to singleton pregnancies $(€ 1489)$ (Wolner-Hanssen and Rydhstroem, 1998; Lukassen et al., 2005). 


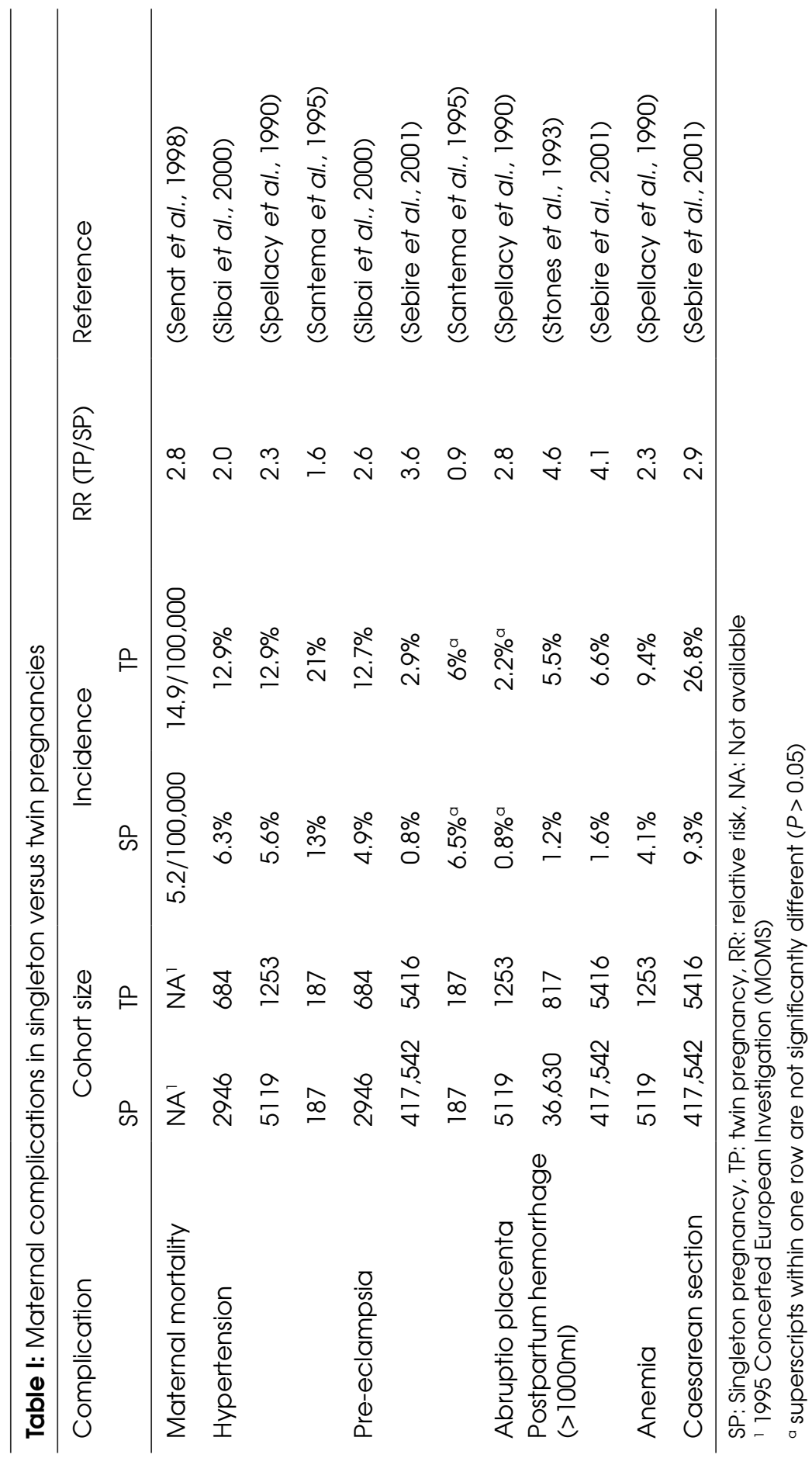




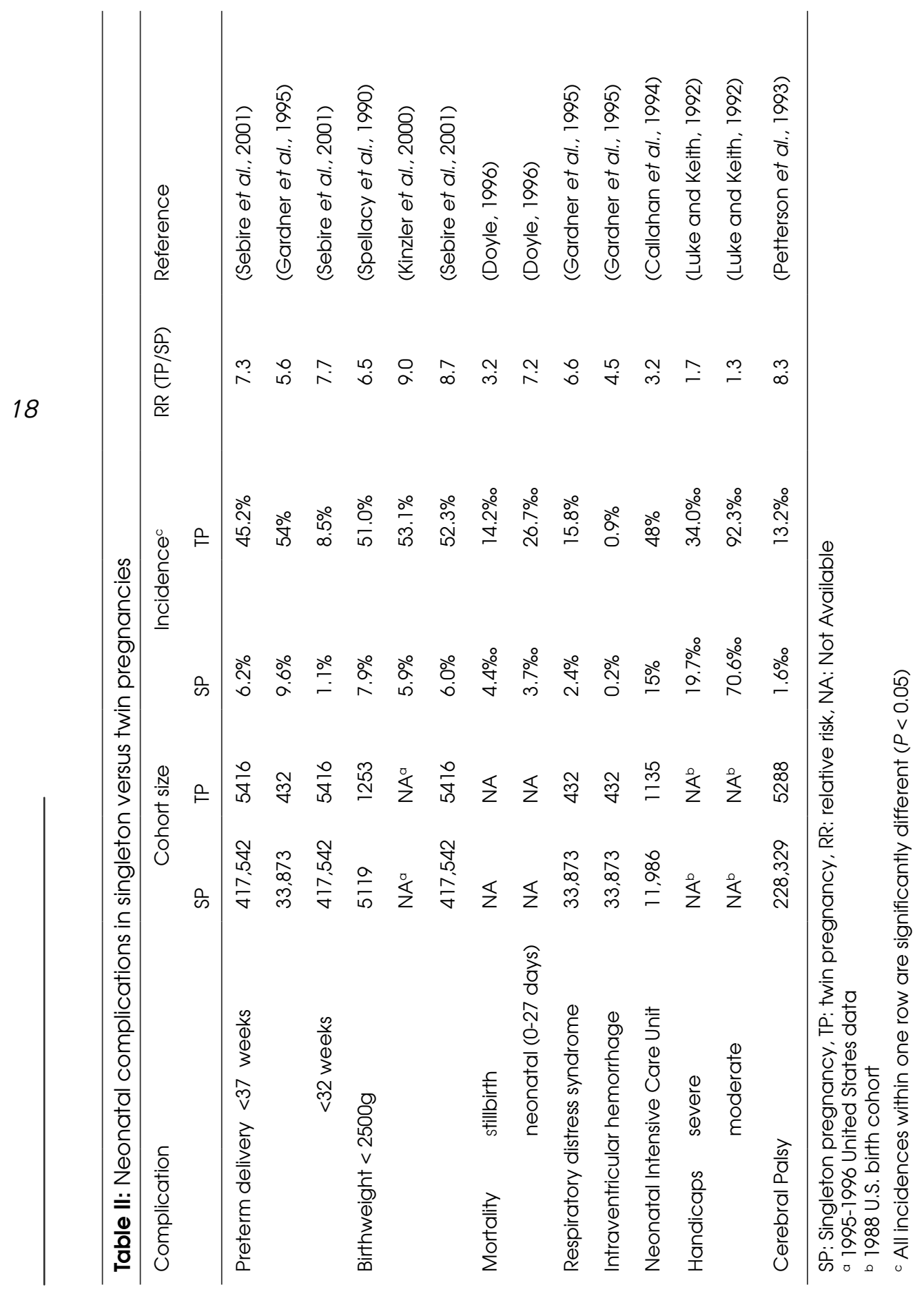




\section{The approach to prevention of twin pregnancies - Single embryo transfer}

In the nineties, when the iatrogenic increase in multiple pregnancies due to multiple embryo transfers was recognized as a complication of IVF, several observational studies focussed on the reduction of the number of embryos transferred. They all found that when reducing the number of transferred embryos from three to two, the multiple PR was reduced without diminishing the overall ongoing PR (Waterstone et al., 1991; Staessen et al., 1993; Fujii et al., 1998; Templeton and Morris, 1998; Dean et al., 2000). The reduction in multiple PR was predominantly due to a virtual elimination of the triplet pregnancies. The rate of twin pregnancies remained constant between 20 and 30\% (Staessen et al., 1993; Fujii et al., 1998; Dean et al., 2000; Nyboe Andersen et al., 2005).

The next step to further reduce the multiple PR (i.e. twin PR) was the transfer of a single embryo (Single Embryo Transfer, SET). In the beginning, SET was applied when there was only one embryo available (compulsory SET, CSET), leading to low PRs of 10.3\% (Giorgetti et al., 1995). But as the overall PR after IVF increased due to the improvement of culture media, a better identification of viable embryos and an improved transfer procedure (Gerris et al., 1999), SET was also introduced in patients who had more than one embryo available (i.e. elective SET, eSET) (Gerris et al., 1999; Vilska et al., 1999).

Gerris and colleagues (1999) were the first to conduct a randomized controlled trial (RCT) between eSET and double embro transfer (DET). Only a selected group of good-prognosis patients, defined by age and number of good morphology embryos was included in the study (Table III). After this study more clinics, especially in Belgium and Finland started with the application of ESET in a selected population of twin prone patiens (Table III and IV) (Gerris et al., 1999; Martikainen et al., 2001). The RCTs performed before the start of our study described in chapter 3 (i.e. before 2002), showed that the transfer of only one good quality embryo resulted in a lower PR as compared with the transfer of two good quality embryos, although the difference was not significant in both studies (Table III). As the PRs after ESET were still acceptable and the twin pregnancies were prevented, it was concluded that eSET should be the transfer policy of choice in this subgroup of good prognosis patients (Gerris et al., 1999; Martikainen et al., 2001)

Vilska et al. (1999) were the first to publish on the influence of applying an ESET policy on the overall outcome in an IVF programme. ESET was performed for medical reasons, risk of OHSS and patient's wish (Table IV). Also Tiitinen et al. (2001) applied eSET only for patient's wish and medical reasons (diabetes mellitus, uterine malformation and indication for prenatal diagnosis). 
The PRs between the eSET and DET group were comparable in both studies, with acceptable overall PRs and slightly reduced twin PRs (Table IV) (Vilska et al., 1999; Tiitinen et al., 2001).

Gerris et al. (2002) showed the results from the preceeding four years, in which the proportion of ESET was gradually increased in their clinic. It started with the RCT in women < 34 years of age in their first IVF or ICSI cycle with at least two top quality embryos (Gerris et al., 1999). After this study a new study was started, in which the criteria for ESET were liberated. Women $<38$ years of age in their first IVF or ICSI cycle were allowed to choose whether they wanted one or two embryos transferred in case a top quality embryo was available. A small subgroup consisted of patients who preferred eSET, irrespective of the availability of a top quality embryo. Due to this judicious implementation of eSET, the overall PR remained similar over the years, while the twin PR was reduced from $29.5 \%$ in 1998 to $16.3 \%$ in 2001 (Gerris et al. , 2002). Also de Sutter et al. (2003) reported a reduction in twin PR (from $30 \%$ to $21 \%$ ) after a gradual implementation of ESET over a four-year period, while the PR (35\%) remained similar.

Despite all efforts in implementing eSET, the twin PR did not fall below 15\%. Although this is a great step forward as compared with the transfer of two embryos (25-30\%), this rate is, in our opinion, unacceptable regarding the risks and complications that are involved in a twin pregnancy. This was also confirmed in the following recommendation made at the ESHRE campus course on the prevention of twin pregnancies: "A twin (and higher order multiple) PR of $25 \%$ or more is not acceptable and must induce practitioners to elaborate an individualized embryo transfer strategy, aiming at reducing this incidence to perhaps around 10\%" (ESHRE Campus Course, 2001).

\section{The approach to prevention of twin pregnancies - Patient and embryo selection for eSET}

\section{Patient selection}

Several studies have focussed on defining predictors for patients at an increased risk for a twin pregnancy and therefore candidates for ESET. Both Strandell et al. (2000) and Hunault et al. (2002) found after a multivariate analysis that female age and number of good quality embryos transferred were the most important factors, independently predictive of birth and multiple birth. Number of previous IVF attempts and duration of infertility were negatively associated with the chance of a birth or a multiple birth (Templeton and Morris, 1998). This was confirmed by Staessen and collegues (1993), who analysed PRs between DET and triple embryo transfer and found no difference in patients $<37$ years old in their first IVF attempt with at least three good quality embryos available for transfer (Staessen et al., 1993). Also Coetsier and Dhont (1998) found that age < 
36 years, first, second or third IVF or ICSI cycle, more than three embryos available for transfer and the quality of the transferred embryos (i.e. at least 4 on a scale of 1-5), are associated with good prognosis.

\section{Embryo selection based on morphologic parameters}

To define the morphological characteristics of a good quality embryo, 23 DETs resulting in a twin pregnancy were analysed. The resulting characteristics are 4 or 5 blastomeres on day 2 , seven or more on day 3 and $\leq$ $20 \%$ fragmentation (Van Royen et al., 1999). Several other studies focussed on the pregnancy predictive value of morphological characteristics of the zygote and embryo at different stages of development. The morphological parameters for the zygote include the number of nuclear precursor bodies (NPB) and their distribution in the pronuclei (Scott and Smith, 1998; Tesarik et al., 2000; Wittemer et al., 2000). Three or more NPBs and an equal distribution in both pronuclei are positively correlated with implantation and blastocyst development. There was only one study analysing SETs, but in this study no significant differences in implantation rate between the zygote scoring classes were found (Salumets et al., 2001). Another parameter analysed, is the time of the first cleavage division of the zygote and the development rate during the forthcomings days. Whether or not the first cleavage division could be seen within 25-27h after insemination or ICSI influenced the pregnancy and implantation rate (Shoukir et al., 1997; Sakkas et al., 1998; Bos-Mikich et al., 2001; Lundin et al., 2001; Sakkas et al., 2001; Fenwick et al., 2002; Tsai et al., 2002). Early cleavage (EC) embryos resulted more often in a pregnancy than non-early cleavage (NEC) embryos. However, most studies analysed multiple embryo transfers of which many cycles had transfers with both an early and a non-early cleavage embryo. Therefore it remains uncertain whether the pregnancy or implantation can be attributed to the early cleavage embryo. At the second day of development, an embryo consisting of four cells with little or no fragmentation has the highest chance to implant (Ziebe et al., 1997; Van Royen et al., 1999). Furthermore, when there are no multinucleated blastomeres (MNBs) (Pelinck et al., 1998; Van Royen et al., 2003) and when the blastomeres are even sized, the implantation potential of the embryo increases even more. Unevenly sized blastomeres might point towards aneuploidy (Hardarson et al., 2001). In clinics transferring at day 3 , the most optimal cell number is 7-8, also with little or no fragmentation and no multinucleated, even sized blastomeres (Van Royen et al., 1999).

\section{Embryo selection based on non-morphologic parameters}

Only the oberservation of embryo morphology on the few days before transfer cannot fully reflect the implantation potential of the embryo. This for instance emerges as around $30-40 \%$ of the morphologically normal embryos and blastocysts display aneuploidy, which reduces the implantation chance (Munne et al., 1995; Magli et al., 2000). 

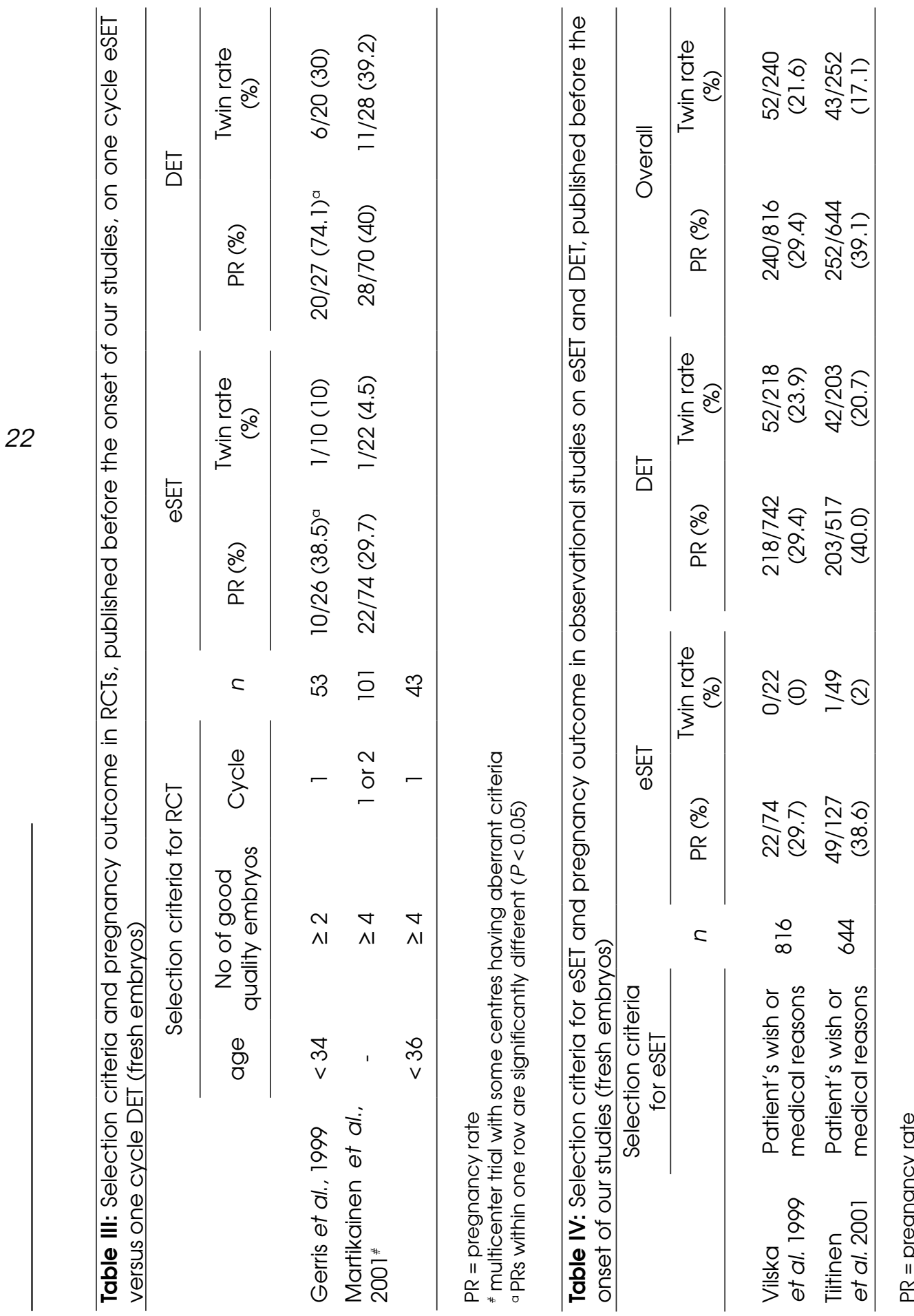
To improve their implantation rate, a new selection method was developed: preimplantation genetic diagnosis on chromosomal aneuploidies (PGDAS). Although a retrospective study in these patients showed a positive effect of PGD-AS on the PRs (Munne et al., 2003), in a large randomized controlled trial no positive effect could be found (Staessen et al., 2004). To further achieve some characteristics of the embyo not visible from the outside, laboratories focus on biochemical markers in culture medium and follicular fluid. To assess the metabolic activity of an embryo, the uptake of nutrients from the surrounding medium or the secretion of metabolites can be examined. Gardner et al. (2001) determined that embryos developing into a blastocyst consumed more glucose and pyruvate and that the quality of the blastocyst was positively related to the glycose uptake. Furthermore, Van den Bergh et al. (2001) showed that blastocysts resulting in a pregnancy had a higher glucose uptake compared to blastocysts not resulting in a pregnancy.

There is increasing evidence that the heterogeneity of the follicular micro-environment has a significant impact on oocyte competence and embryonic development. Follicular fluid obtained after oocyte retrieval can be analysed for several factors. It appeared that the concentration of hormones (17 $\beta$-estradiol, LH, growth hormone, prolactin), growth factors (insulin-like growth factor-I) and cytokines (interleukin-1) in follicular fluid are correlated with pregnancy (Mendoza et al., 2002). Also ATP content of the oocyte and dissolved oxygen content of the follicle fluid have been suggested as parameters for oocyte/embryo development (Van Blerkom et al., 1995; Van Blerkom et al., 1997).

Besides the biochemical markers of the intrafollicular environment, also vascularization of the follicles has been examined as a potential parameter for embryo quality. The vascularization of pre-ovulatory follicles is related to oocyte oxygenation (Van Blerkom et al., 1997) and can differ between the follicles of one patient. Nargund et al. (1996) found by Doppler imaging of the follicular blood flow that oocytes from poorly vascularized follicles developed in morphologically inferior embryos as compared to well vascularized follicles. Several other studies confirmed a positive relationship between perifollicular vascularization and pregnancy (Chui et al., 1997; Van Blerkom et al., 1997; Borini et al., 2004). Pregnancies were only achieved with embryos from follicles where vascularity was detected in $>50 \%$ of the follicular circumference and live births only from oocytes with $>76 \%$ follicular vascularity (Chui et al., 1997). In addition, both Chui et al. (1997) and Van Blerkom et al. (1997) reported a significant higher incidence of aneuploidy and spindle defects in oocytes derived from follicles with poor vascularization as compared to well-vascularized follicles. 


\section{Aim and outline of thesis}

As a twin pregnancy is one of the most adverse outcomes of an IVF treatment, affecting around $25 \%$ of the patients, the studies described in this thesis focussed on the reduction of the twin PR after IVF. The problem of and possible solutions for the prevention of twin pregnancies will be viewed from different angles. The general aim of the thesis was to analyse several transfer strategies with ESET from a clinical and a cost-effectiveness point of view and to improve the selection of the single best embryo for ESET.

In a first attempt to reduce the twin PR, the influence of eSET applied in all cycles, instead of only the first one, in a selected group of good prognosis patients was analyzed (chapter 2). To further reduce the twin PR without gradually liberating the selection criteria, ESET was applied in the first cycle of an unselected group of patients. The clinical outcome as well as the cost-effectiveness of this strategy was analysed by performing an RCT of ESET versus DET (chapter 3 and 4). Finally, another strategy to reduce the twin PR, the application of eSET in the first cycle of all patients and ESET or DET depending on patient and embryo characteristics in the following cycles, was analysed (chapter 5).

To optimize PRs after IVF, and especially after eSET, the embryo with the highest implantation potential has to be selected. This selection was improved by focussing on early cleavage (chapter 6). Since also intrafollicular factors influence embryo development, the final objective of this thesis was to study whether differences in follicular gene expression reflect embryo viability (chapter 7). 
CHAPTER 2

\section{Elective single embryo transfer (eSET) policy in the first three IVF/ ICSI treatment cycles}

Aafke P.A. van Montfoort' , John C.M. Dumoulin', Jolande A. Land², Edith Coonen', Josien G. Derhaag' and Johannes L.H. Evers².

Research Institute Growth \& Development (GROW), University of Maastricht; IVF-Laboratory' and Department of Obstetrics\& Gynaecology'2, Academic Hospital Maastricht, Maastricht, The Netherlands

Published in: Hum Reprod (2005), 20, 433-436. 


\begin{abstract}
BACKGROUND: Elective single embryo transfer (eSET), applied in the first or second IVF cycle in young patients with good quality embryos, has been demonstrated to lower the twin pregnancy rate (PR), while the overall PR is not compromised. It is as yet unclear whether ESET could be the preferred transfer policy in all treatment cycles, or that it should be restricted to the first or first two cycles.

METHODS: eSET policy (when two or more embryos were available, at least one of them being of good quality) was offered to patients younger than 38 years in the first three treatment cycles. Retrospectively, treatment cycle outcome was studied.
\end{abstract}

RESULTS: In 326 patients, 586 treatment cycles were performed (326 first, 168 second and 92 third treatment cycles). In 65 cycles (11\%), eSET could not be applied because there was either no fertilization, or only one embryo available. In the remaining 521 cycles, eSET was performed in 111 cycles (19\%), while in 410 cycles, no good quality embryo was available resulting in the transfer of two embryos (double embryo transfer, DET). No significant differences in ongoing PRs after transfer of fresh embryos were observed between eSET and DET in the first (both 33\%), second (36 and $23 \%$, respectively) and third treatment cycles (20 and $24 \%$, respectively). In significantly more eSET cycles compared to DET cycles, embryos could be frozen. This resulted in a significantly higher cumulative PR after ESET compared to DET.

CONCLUSION: In patients younger than 38 years with at least one top quality embryo, eSET can be the transfer policy of choice in at least the first three treatment cycles, since the PRs obtained in each treatment cycle are comparable to those after DET. 


\section{Introduction}

Multiple pregnancy rates (PRs) are high in assisted reproductive technology (ART) cycles. Recently, the European IVF-monitoring programme of the European Society of Human Reproduction and Embryology (ESHRE) reported a $26.3 \%$ multiple delivery rate in $258,460 \mathrm{IVF} / \mathrm{ICSI}$ treatment cycles initiated during 1999 in 22 countries (ESHRE, 2002). The majority of multiple deliveries after IVF and ICSI concern twins, representing $24.0 \%$ of all deliveries (ESHRE, 2002). Twin pregnancies must be regarded as a serious complication of ART cycles, with relatively high risks of health problems in the children (both during the pre- and perinatal period and later in life) and their mothers, social problems, and high economic costs (ESHRE Capri Workshop, 2000; Gerris et al., 2004).

The high twin PR after IVF is the result of the current standard practice of transferring more than one embryo. The elective transfer of only a single embryo (eSET) has been shown to be an effective method to reduce the incidence of twin pregnancies in twin-prone IVF/ICSI patients without compromising the overall ongoing PR (Vilska et al., 1999; ESHRE Campus Course, 2001; Gerris et al., 2001; Martikainen et al., 2001; Tiitinen et al., 2001; Gerris et al., 2002; De Neubourg and Gerris, 2003; Tiitinen et al., 2003).

It was recently recommended by the ESHRE consensus meeting on risks and complications in ART that eSET should be proposed in the first and second treatment cycles (Land and Evers, 2003). Since 2003 in Belgium, SET in the first, and ESET in the second treatment cycle is mandatory in patients younger than 36 years of age, to obtain maximal reimbursement of the costs of IVF treatment (Ombelet, 2004). However, little information is available on eSET results to substantiate this proposal to limit eSET to the first two treatment cycles. In the present study we evaluate the results per treatment cycle number in a cohort of patients in whom eSET was offered as the standard transfer policy during the first three treatment cycles.

\section{Materials and methods}

\section{Patients}

All IVF/ICSI patients who started their first treatment cycle in the period from July 2000 until December 2001 in the academic hospital Maastricht were included in this study. A treatment cycle was defined as an ovarian stimulation cycle which resulted in an ovum pick-up. In the study period ESET was offered as our standard transfer policy (see below) to patients younger than 38 years at the time of transfer, irrespective of the rank of the cycle and of previous IVF results. Only patients who were 38 years or older, patients with a strong wish for the transfer of either one or two embryos, patients who had a medical or socio/psychological reason to prevent 
a twin pregnancy, and patients requesting preimplantation genetic diagnosis (PGD) were excluded from the standard eSET policy.

In the study period, the costs of the first three IVF/ICSI treatment cycles were fully reimbursed in The Netherlands. For this reason the study has been limited to the first three treatment cycles, including the transfer of frozen embryos, if any.

\section{Ovarian stimulation protocol}

Patients were down-regulated with daily s.c. injections of triptorelin (Decapeptyl; Ferring B.V., Hoofddorp, The Netherlands) or nafarelin intranasally (Synarel; Searle BV, Maarssen, The Netherlands) according to the long protocol. To stimulate multiple follicular development, recombinant FSH (Puregon, Organon, Oss, The Netherlands) was used. Follicle growth was monitored by ultrasound and 5000 IU of hCG (Pregnyl, Organon, Oss, The Netherlands) was given as soon as at least three follicles were $\geq 18 \mathrm{~mm}$. Ultrasound-guided oocyte retrieval was performed $36 \mathrm{~h}$ after hCG administration. The luteal phase was supported by progesterone (Progestan, Organon, Oss, The Netherlands) 600 mg daily intravaginally, starting at the day of ovum pick-up and continued for 14-16 days. In the case of pregnancy $600 \mathrm{mg}$ Progestan was continued for three more weeks.

\section{Laboratory and embryo transfer procedures}

IVF and ICSI procedures used have been described in detail earlier (Dumoulin et al., 2001). Oocytes and embryos were cultured individually in $5 \mu \mathrm{l}$ droplets covered by mineral oil in an atmosphere of $5 \% \mathrm{O}_{2}, 5 \% \mathrm{CO}_{2}$ and $90 \% \mathrm{~N}_{2}$ in sequential culture media either from Vitrolife (Göteborg, Sweden) or from Cook (Eight Mile Plains, Queensland, Australia). Embryo transfer was routinely performed on day two or day three after ovum retrieval. Embryos were evaluated at 23-26h post-injection (in case of ICSI) or 25-28h post-insemination (in case of IVF), 41-45h post-injection/ insemination and in case of day three transfer also at 65-69h post-injection/ insemination. For each embryo originating from a normally fertilized oocyte, an embryo score was calculated on the basis of morphological grade ( 1 to 4 , with grade 4 being the best grade, using the grading system of Bolton et al. (1989)), number of blastomeres and presence or absence of multinucleated blastomeres (MNBs). Embryos that had reached the 4or 5-cell stage on day 2 or the 8-cell stage on day 3 , in combination with having the best morphological grade (regular, even sized blastomeres with $<20 \%$ fragmentation) and an absence of MNBs were classified as good quality embryos (Van Royen et al., 1999). When patients were eligible for standard ESET policy and if at least one good quality embryo was available, only a single embryo was transferred (eSET). In all other cases, two embryos, if available, were transferred (double embryo transfer, DET). Cryopreservation of supernumerary embryos was performed on the 
third day after ovum retrieval. Only embryos which had reached the 8cell stage and which were considered to be of sufficient morphological quality (grades 3 or 4 ) were cryopreserved. After thawing, two embryos, if available, were transferred, also in cases in which in the fresh transfer eSET was applied.

\section{Pregnancy}

An hCG pregnancy test with a detection limit of $50 \mathrm{lU} / \mathrm{l}$ in urine was performed 14-16 days after embryo transfer and patients with a positive test had an ultrasound examination 3 weeks later. Patients were asked to report an abortion occuring after this ultrasound examination immediately. In this study an ongoing pregnancy was defined as the presence of at least one intrauterine gestational sac with fetal heartbeat on ultrasound at $\sim 7 / 8$ weeks gestation and no report of pregnancy loss before 12 weeks gestation.

\section{Statistics}

The $\chi^{2}$ test with Bonferroni correction was used to compare the PRs. Differences were considered significant at $P<0.05$.

\section{Results}

In our IVF center, in the period of July 2000 until December 2001, 388 patients underwent their first IVF/ICSI treatment cycle. The results of 62 patients (16\%) were not included in the present study for the following reasons: 15 patients applied for PGD, 43 patients had reached the age of 38 years at the time of their first or subsequent IVF treatment cycles and four patients had either a strong wish for the transfer of one or two embryos or had medical or socio/psychological reasons for single embryo transfer.

The results of the first treatment cycles (performed in the period July 2000 until December 2001) and of all eventual subsequent second or third treatment cycles or frozen embryo replacement cycles (performed in the period July 2000 until May 2004) of the 326 patients who accepted our standard eSET policy can be found in Table I. In these patients, after transfer of fresh embryos, 159 ongoing pregnancies were achieved (49\%), of which 40 (25\%) were twin pregnancies. In 175 patients (54\%), an ongoing pregnancy was achieved, either after transfer of fresh embryos in the first, second or third treatment cycle, or after transfer of cryopreserved embryos.

The 326 patients eligible for eSET policy underwent 586 treatment cycles ( 1.8 cycle per patient). Total fertilization failure was found in 26 cycles ( $4 \%$ ), while in 39 cycles (7\%), only one embryo was obtained and transferred 
(compulsory SET, CSET). In 521 treatment cycles, more than one embryo was available. In 111 of these cycles (21\%), at least one good-quality embryo was present and consequently only one embryo was transferred (eSET). In this group, 39 positive pregnancy tests (35\%) were achieved, of which four ended in an early abortion (10\%). In 410 cycles, two embryos were transferred (DET). In this group 142 positive pregnancy tests (35\%) were achieved, of which 23 ended in an early abortion (16\%). The PRs after one, two or three cycles were respectively 33,36 and $20 \%$ for eSET and 33,23 and $24 \%$ for DET. The differences between the first, second and third ESET cycle and between the first, second and third DET cycle were not statistically significant. Neither are the differences between ESET and DET in each treatment cycle rank group.

In 112 cycles at least one surplus embryo of good morphology was available after transfer and subsequently frozen. After eSET significantly more often embryos could be cryopreserved as compared to after DET ( $53 \%$ and $13 \%$ of the treatment cycles, respectively). In table I, pregnancy results are shown for 65 thaw cycles performed in patients who did not get pregnant in any of their transfers of fresh embryos. This resulted in 16 ongoing pregnancies (25\%). In 12 patients, thawing has not yet been performed because of the patients' wishes. The cumulative ongoing PRs after fresh and frozen transfers were significantly different between ESET and CSET and between eSET and DET (13\% CSET, $41 \%$ ESET and 30\% DET). An additional 35 patients who became pregnant from fresh embryos, had embryos cryopreserved. In seven of these patients embryos have been thawed, resulting in two pregnancies.

After the first cycle, 48 patients (30\% of the patients not getting pregnant in the first cycle) dropped out because of medical reasons or patient wishes. After the second cycle the drop-out rate was 36\% (27 patients). The Dutch health insurance system only reimburses three IVF/ICSI cycles. Therefore the drop-out rate after the third cycle increased to $75 \%$. 


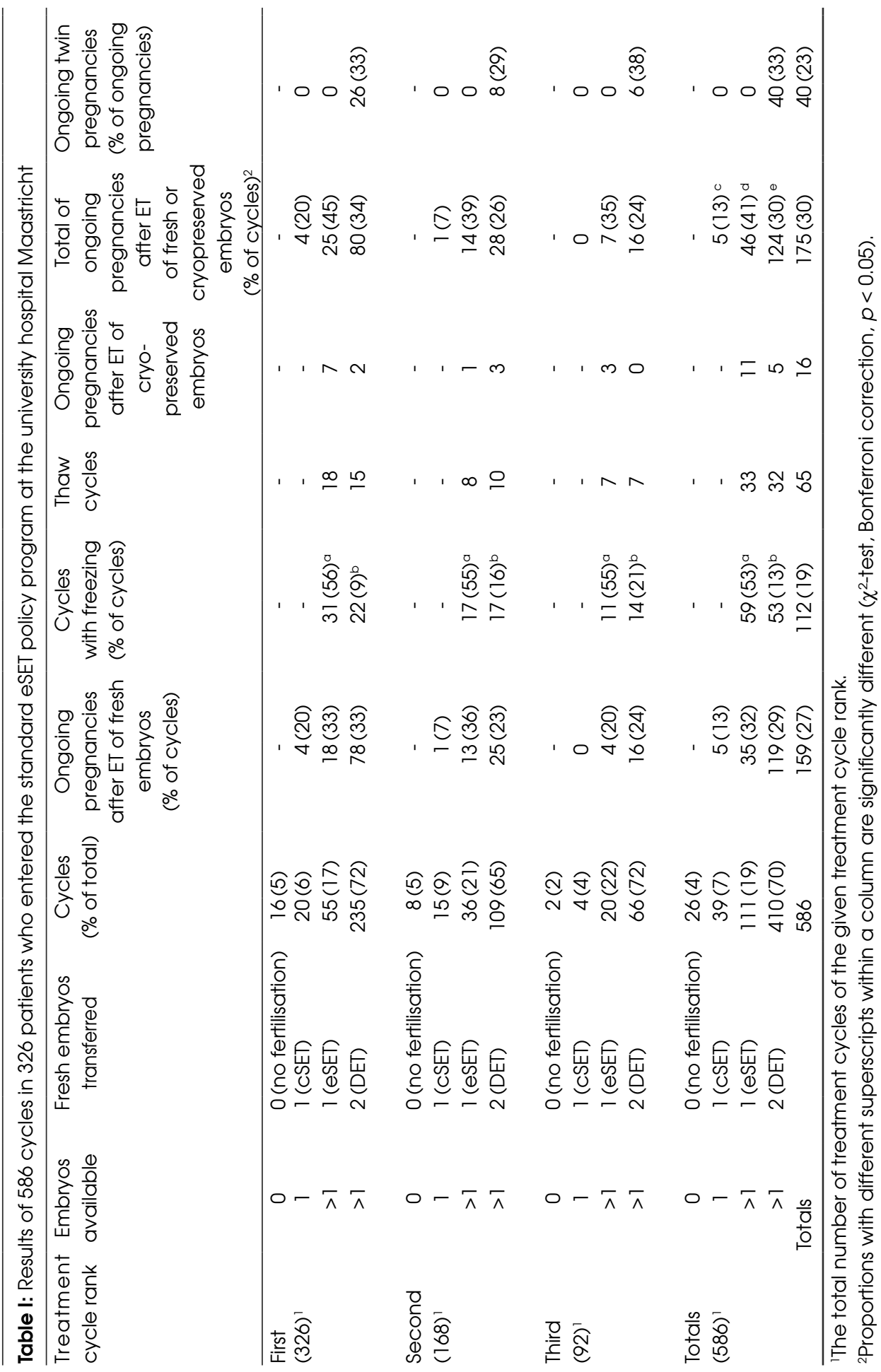




\section{Discussion}

After publication of the encouraging results from the first eSET studies (Gerris et al., 1999; Vilska et al., 1999) showing that acceptable PRs can be obtained when ESET is carried out in a subset of good prognosis patients, and after a brief pilot study in our own clinic, we felt confident in introducing eSET in the year 2000 as our standard clinical ET policy. ESET was offered to all patients younger than 38 years of age, who had at least one good quality embryo, irrespective whether it was their first, second or third treatment cycle.

The PRs obtained in the first two treatment cycles in our study are comparable to those reported by other studies in which ESET was performed in either only the first (Gerris et al., 1999; Vilska et al., 1999) or in the first two treatment cycles (Martikainen et al., 2001; De Neubourg and Gerris, 2003). It is concluded in several publications that ESET should be limited to the first two cycles (Martikainen et al., 2001; De Neubourg and Gerris, 2003; Land and Evers, 2003). However, our results show that PRs of eSET compared to DET are not significantly different up to and including the third treatment cycle, although it must be noted that the group of patients that received a third treatment cycle is relatively small (four pregnancies in 20 patients receiving only a single embryo). Yet, the PRs in the third treatment cycle in both the ESET and the DET groups are decreased compared with the previous treatment cycles. These decreasing PRs in successive IVF cycles were already reported by others (Tan et al., 1992; Templeton et al., 1996). Our results confirm earlier publications showing that cryopreservation clearly improves the cumulative PR after eSET. (Martikainen et al., 2001; Tiitinen et al., 2001). In our study, in significantly more eSET cycles could embryos be cryopreserved compared to DET cycles (53 and 13\% respectively). After thawing, two embryos, if available, were transferred. The reason for this was that cryopreserved embryos have a lower potential for implantation and therefore were not considered to be 'good quality' embryos, which is a necessary condition for eSET (Levran et al., 1990; Edgar et al., 2000a; Edgar et al., 2000b). At present, almost all embryos, from patients who did not became pregnant from fresh embryos, have been thawed. A significantly higher cumulative PR in the eSET group can be seen, caused by the additive effect of frozen embryo transfers.

In order to improve the results after eSET, effort is taken to improve the embryo selection criteria. In addition, to further reduce the risk of twins, the proportion of eSET transfers needs to be enlarged. Tiitinen et al. (2003) reported an increase over the years in the proportion of eSET in their IVF/ ICSI programme from $10 \%$ to $56 \%$, while maintaining comparable PRs. The same applies for Gerris et al., who reported an increase of eSET from $9.8 \%$ in 1998 to $23.6 \%$ in the year 2001 (Gerris et al., 2002). This increase was achieved by liberalization of the criteria for application of eSET. However, 
the twin PR in both clinics (ranging from $7.5 \%$ to 13.5\%) (Tiitinen et al., 2003; Gerris et al., 2004) remained above the twin PR after a spontaneous pregnancy (ranging from 1 to 1.5\%) (Ghai and Vidyasagar, 1988; Dhont, 2001). In the present study, $19 \%$ of the patients eligible for the eSET policy received one embryo. In the DET group however, a high twin PR (23\%) was found in spite of "suboptimal" quality embryos. Apparently our embryo selection criteria for eSET are still too strict. Instead of gradually liberating the criteria for ESET, we decided to start a prospective randomized study in which consenting patients will be allocated by lot for the transfer of either one or two embryos, irrespective of the presence or absence of a top quality embryo. This study will provide more insight into whether we should perform SET in every patient, or how the selection of the embryos and the patients suitable for SET can be improved.

In conclusion, eSET in a selected group of patients (younger than 38 years with at least one good quality embryo) can be performed not only in the first two treatment cycles, but also in the third cycle while maintaining a PR comparable to DET. In this way the proportion of ESET in the total IVF programme can be increased, which will result in a further decline in the twin PR. 


\section{In unselected patients, elective single embryo transfer prevents all multiples, but results in significantly lower pregnancy rates as compared with double embryo transfer; a randomized controlled trial}

Aafke P.A. van Montfoort' , Audrey A.A. Fiddelers², J. Marij Janssen', Josien G. Derhaag', Carmen D. Dirksen², Gerard A.J. Dunselman', Jolande A. Land' ', Joep P.M. Geraedts ${ }^{3}$,Johannes L.H. Evers', John C.M. Dumoulin ${ }^{1}$

Research Institute Growth \& Development (GROW), 'Department of Obstetrics \& Gynaecology, ${ }^{2}$ Department of Clinical Epidemiology and Medical Technology Assessment, and ${ }^{3}$ Department of Clinical Genetics, Academic Hospital Maastricht, The Netherlands

Published in: Hum Reprod (2006), 21, 338-343. 


\begin{abstract}
BACKGROUND: Elective single embryo transfer (eSET) in a selected group of patients (i.e. young patients with at least one good quality embryo) reduces the number of multiple pregnancies in an IVF programme. However, the reduced overall multiple pregnancy rate (PR) is still unacceptably high. Therefore, a randomized controlled trial (RCT) was conducted comparing ESET and double embryo transfer (DET) in an unselected group of patients (i.e. irrespective of woman's age or embryo quality).
\end{abstract}

METHODS: Consenting unselected patients were randomized between eSET (RCT-eSET) $(n=154)$ or DET (RCT-DET) $(n=154)$. Randomization was performed just prior to the first embryo transfer, provided that at least two 2PN zygotes were available. Non-participants received our standard transfer policy (SP-eSET in a selected group of patients $(n=100)$, otherwise SP-DET $(n=122)$ ).

RESULTS: The ongoing PR after RCT-eSET was significantly lower as compared with RCT-DET (21.4\% versus $40.3 \%$ ) and the twin PR was reduced from $21.0 \%$ after RCT-DET to $0 \%$ after RCT-eSET. The ongoing PRs after SP-eSET and SPDET did not differ significantly (33.0\% versus $30.3 \%$ ), with an overall twin PR of $12.9 \%$.

CONCLUSION: To avoid twin pregnancies resulting from an IVF treatment, ESET should be applied in all patients. The consequence would be a halving of the ongoing PR as compared with applying a DET policy in all patients. The transfer of one embryo in a selected group of good prognosis patients leads to a less drastic reduction in PR but maintains a twin PR of $12.9 \%$. 


\section{Introduction}

A multiple pregnancy is a serious adverse outcome of an IVF treatment (Land and Evers, 2003). In many (European) IVF centres, the standard embryo transfer policy is to transfer two embryos (Nyboe Andersen et al., 2004). However, although higher order multiple pregnancies are reduced to $2 \%$ on average, the twin pregnancy rates (PRs) remain between 20 and 35\% (Nyboe Andersen et al., 2004). Twin pregnancies should also be considered as a serious disadvantage, not only because of the increased risks of medical and perinatal complications (ESHRE Capri Workshop, 2000; Helmerhorst et al., 2004), but also because of the increased health care costs associated with enhanced pre- and postnatal care (De Sutter et al., 2002; Gerris et al., 2004; Lukassen et al., 2004). The only way to solve this problem is to reduce the number of embryos transferred to one.

Several studies have investigated elective single embryo transfer (eSET). At least five randomized controlled trials (RCTs) have been published (Gerris et al., 1999; Martikainen et al., 2001; Gardner et al., 2004; Thurin et al., 2004; Lukassen et al., 2005). In these studies, only patients at risk for a twin pregnancy were randomized between the transfer of one or two good quality embryos (double embryo transfer; DET). The selection criteria for patients at risk varied between the studies, but were based on female age ( < 34 years, Gerris et al. (1999); < 35 years, Lukassen et al. (2005) and some of the patients in the study of Thurin et al. (2004); < 36 years, Martikainen et al. (2001) and some of the patients in the study by Thurin ef al. (2004)), and the number of good quality embryos available ( $\geq 2$ in the studies of Gerris et al. (1999), Thurin et al. (2004) and Lukassen et al. (2005), $\geq 3$ in some of the patients in the study of Thurin et al. and $\geq 4$ in one of the participating centres in the study by Martikainen et al. (2001)). Furthermore, no previous failed cycles were allowed in some of the studies (Gerris et al. (1999), Lukassen et al. (2005)). These studies showed that the transfer of only one good quality embryo resulted in lower PRs as compared with the transfer of two good quality embryos, although the differences were not significant in all studies. However, as PRs after ESET were acceptable and twin pregnancies were avoided, it was concluded by the authors that ESET should be the transfer policy of choice in this subgroup of patients. Furthermore, in two RCTs, it was demonstrated that the difference in PRs between SET and DET can be overcome by performing one additional SET cycle (Lukassen et al., 2005) or a frozen embryo transfer cycle if frozen embryos from the fresh cycle were available (Thurin et al., 2004).

Besides the RCTs mentioned, several observational studies have been published (Tiitinen et al., 2001; Gerris et al., 2002; Van Montfoort et al., 2005) in which the results of eSET applied in a selected group of good prognosis patients (20-30\% of the IVF population) were compared with the results of DET applied in the remaining group of patients. These studies concluded that ESET and DET resulted in comparable PRs and that the 
overall twin PR in an IVF programme could be reduced considerably. In the most recent studies, $55-60 \%$ of all patients was offered eSET, while DET was offered to the remaining patients (Tiitinen et al., 2003; Gerris et al., 2004). This resulted in an overall twin PR of $~ 10 \%$. Although this rate is significantly lower than the rate obtained with a transfer policy consisting of only DET (20-35\%), it is still substantially higher than the rate in spontaneous pregnancies. From the studies published so far, it can be concluded that ESET offers an acceptable PR with a low twin PR in a selected group of patients. It is unknown whether PRs remain acceptable if eSET is applied in an unselected group of patients, thereby reducing the twin PR to a value comparable with the spontaneous twin PR. Therefore, a prospective RCT was conducted where patients were assigned to either ESET or DET irrespective of their age (within the age limits applied in our IVF programme) and irrespective of whether or not a good quality embryo was available.

The primary aim of this study was to compare the PRs in both study groups. The RCT was limited to the first cycle of patients and to the transfer of fresh embryos only. A secondary aim of this study was to evaluate PRs after eSET and DET when the decision of whether to transfer one or two embryos was based on female age ( $<38$ years) and the presence of at least one good quality embryo.

\section{Materials and methods}

\section{Patients and study design}

From January 2002 until December 2004, patients who started their first IVF cycle in the academic hospital of Maastricht, the Netherlands, were assessed for eligibility to participate in the study. Patients applying for preimplantation genetic diagnosis (PGD), patients requiring the transfer of only one embryo (in most cases because of medical reasons), and patients who could not be informed adequately because of a language barrier were excluded. All other patients were informed about the study, including the possibility of a lower PR after ESET and the pre- and postnatal risks of twins. Eligible patients choosing not to participate in the RCT were offered our standard transfer policy (see below).

Consenting patients had to have normal fertilization of at least two oocytes (i.e. 2PN embryos) in order to be randomized between eSET (referred to as the RCT-eSET group) and DET (referred to as the RCT-DET group). Randomization was performed immediately prior to embryo transfer. To ensure comparability between ESET and DET with respect to female age ( $<38$ years or $\geq 38$ years) and fertilization technique (IVF or IVF/ICSI), the patient population was stratified with respect to these four characteristics. Furthermore, to avoid confounding by fluctuations of success rates over time, the groups were subdivided into smaller groups to ensure an equal distribution of ESET and DET over a time-period (an 
average of 3 months). By varying the size of these subgroups (ranging from 8 to 14) and by using a non-transparent box containing the sealed opaque envelopes, the randomization procedure was blinded. The laboratory personnel performing the randomization were unaware of the size of the subgroups.

After transfer, patients were informed about the number of embryos transferred. Any subsequent IVF or IVF/ICSI cycle and all transfer cycles of cryopreserved embryos were not a part of the RCT. In these cycles our standard transfer policy was applied. The standard transfer policy in our clinic consisted of the transfer of a single embryo when female age was $<38$ years and at least one good-quality embryo (see below) was available. Otherwise, two embryos were transferred. In order to compensate for any possible disadvantage due to a lower PR in the RCT-eSET group of the study, these patients were offered a fourth IVF or IVF/ICSI cycle free of charge if pregnancy was not achieved in the first three cycles which, as a rule, were covered by the national health system or by private insurance companies in the Netherlands.

To address the secondary aim of our study, data from patients who received the standard transfer policy in their first treatment cycle were used. This group was composed of all patients eligible for the study, but not willing to participate and the patients not eligible for the study because of a language barrier (non-participants group). Furthermore, in order to compare eSET (referred to as the standard policy eSET group, SPeSET) with DET (SP-DET group), at least two normally fertilized oocytes (2PN zygotes) had to be present. The study was approved by the Institutional Ethical Board of the academic hospital of Maastricht and all participating patients in the RCT signed an informed consent. The non-participating patients signed an informed consent for the use of their data.

Sample size calculation

To calculate sample size for the RCT part of the study, data from a previous period in our IVF clinic, in which DET was our standard policy, were used. An ongoing PR of $29 \%$ was achieved after DET. Assuming a similar ongoing PR for the DET group in the study and considering an ongoing PR in the ESET group of $<15 \%$ as clinically unacceptable, the required sample size was 150 cycles in both the eSET and DET study group with a power of $80 \%$ and an $\alpha$ of 0.05 .

\section{Ovarian stimulation protocol}

Patients were downregulated with $0.1 \mathrm{mg}$ triptorelin daily s.c. (Decapeptyl; Ferring B.V., Hoofddorp, The Netherlands) according to the long protocol. To stimulate multiple follicular development, recombinant FSH (Puregon, Organon, Oss, The Netherlands) was used. Follicle growth was monitored by ultrasound and $5000 \mathrm{IU}$ of hCG (Pregnyl, Organon, Oss, The Netherlands) was administered as soon as at least three follicles were $\geq 18 \mathrm{~mm}$. Ultrasound- 
guided oocyte retrieval was performed $36 \mathrm{~h}$ after $\mathrm{hCG}$ administration. The luteal phase was supported by progesterone (Progestan, Organon) 200 mg three times daily intravaginally, starting at the day of ovum pick-up and continued for 14-16 days. In case of a pregnancy, progesterone was continued for another 3 weeks.

Culture procedure, embryo quality assessment and transfer policy

IVF, ICSI and embryo culture procedures have been described in detail earlier (Dumoulin et al., 2000). For each embryo originating from a normally fertilized oocyte, an embryo score was calculated on the basis of morphological grade (1-4, with grade 4 being the best grade), number of blastomeres and presence or absence of multinucleated blastomeres (MNBs) (Van Montfoort et al., 2005). Embryos that had reached the 4- or 5 -cell stage on day 2 , or the 8 -cell stage on day 3 , in combination with having the best morphological grade (regular, even sized blastomeres with less than $20 \%$ fragmentation) and an absence of MNBs were classified as good quality embryos (Van Montfoort et al., 2005). Embryos were transferred on day 2 after ovum pick-up or, in a minority of cases, for reasons of convenience, on day 3 . In all cases, including those in the RCT study, embryos with the highest embryo score were transferred.

Cryopreservation of supernumerary embryos was performed on the morning of the third day after ovum pick-up if one or more embryos had reached the 8-cell stage, and if they were of good morphological quality.

\section{Outcome variables}

Primary outcome variables were ongoing PR and twin PR at 10 weeks after ovum pick-up ( 12 weeks gestational age). An hCG pregnancy test with a detection limit of $50 \mathrm{IU} / \mathrm{I}$ in urine was performed 14-16 days after embryo transfer, and patients with a positive test had an ultrasound examination 3 weeks later. An ongoing pregnancy was defined as the presence of at least one intrauterine gestational sac with fetal heartbeat on ultrasound at 7 weeks gestation, and no report of pregnancy loss when patients were contacted at 12 weeks gestation.

\section{Statistics}

Analysis of variance (ANOVA) with Tukey's multiple test procedure was used to compare the continuous variables and the $\chi^{2}$ test with Bonferroni correction was used for binary variables. A $P$ value $<0.05$ was considered significant. 


\section{Results}

Of the 807 patients who visited the clinic for their first IVF or IVF/ICSI cycle in the period from January 2002 until December 2004, 133 (16.5\%) patients did not meet the inclusion criteria (PGD $(n=72)$, eSET for medical reasons $(n=42)$ or language barrier $(n=19))$. Furthermore, $53(6.6 \%)$ patients refrained from IVF treatment or achieved a spontaneous pregnancy while waiting for the start of their first IVF cycle. Of the 621 eligible patients, 348 (56\%) agreed to participate in the RCT part of the study. Of these, 40 patients (11\%) could not be randomized because of fertilization failure or because only one embryo was available. The remaining 308 patients were randomized immediately prior to embryo transfer: 154 patients received one embryo and 154 received two (Figure 1). In 123 (80\%) patients in the SET group and 116 patients (75\%) in the DET group, the transfer was performed on day 2. The remaining patients received their embryos on day 3.

Patient and cycle characteristics were comparable between the two study groups of the RCT (Table I). When the clinical outcomes of the eSET and DET group of the RCT were compared, the percentage of positive pregnancy tests after transfer of fresh embryos differed significantly between eSET and DET (33.1 versus 47.4\%, respectively) (Table II). In addition, the abortion rate was significantly higher after eSET as compared with DET (35.3 versus 15.1\%), resulting in a doubling of the ongoing PR after DET as compared with eSET (40.3 versus $21.4 \%$ ). The twin PR after eSET was reduced to $0 \%$, whereas $21.0 \%$ of the ongoing pregnancies after DET were twin pregnancies (Table II).

In the non-participants group ( $n=292$, composed of 273 patients who declined to participate in the RCT and 19 otherwise eligible patients with a language barrier), in 70 patients the standard transfer policy could not be applied because of the following reasons: a) cancellation of the cycle before ovum pick-up $(n=28)$, b) no fresh embryo transfer and cryopreservation of all embryos was performed because of

ovarian hyperstimulation syndrome (OHSS) $(n=3), c)$ total fertilization failure was found $(n=11)$ or $d)$ only one embryo was obtained and transferred (compulsory SET) $(n=28)$. The remaining 222 patients had at least two fertilized embryos and were suitable for a comparison between eSET and DET according to our standard transfer policy (SP-eSET and SP-DET). SPeSET was applied in $45 \%$ of the patients (Table III). Patient characteristics from the non-participants group were similar to those of the participants in the RCT study except for the mean age (Table I). The ongoing PRs in the SP-eSET and SP-DET groups were $33.0 \%$ and $30.3 \%$, respectively (Table III). The overall ongoing PR with the standard transfer policy was $31.5 \%$ and the overall twin PR was $12.9 \%$. 


\section{Discussion}

During the last few years ESET has become more and more accepted as the solution for the high multiple PR after IVF and IVF/ICSI. Until now, ESET was only applied in a selected group of patients. In this study, an RCT was performed in the first cycle of an unselected group of patients, i.e. irrespective of female age (within the age limits applied in our IVF programme) and irrespective of the availability of a good quality embryo. The transfer of one embryo in this unselected group resulted in a significantly lower PR (21.4\%) than the transfer of two embryos (40.3\%). This tendency is also seen in previously performed RCTs, which were conducted in a subset of good prognosis patients. In these studies, the ongoing PRs after eSET and DET were 38.5 versus $74.0 \%$ (Gerris et al., 1999) and 28.5 versus $44.1 \%$ (Thurin et al., 2004), and the live birth rates after ESET and DET were 29.7 versus $40.0 \%$ (Martikainen et al., 2001) and 26 versus 36\% (Lukassen et al., 2005), respectively. In all the aforementioned studies, in selected, good prognosis patients as well as in non-selected patients (present study), the transfer of two embryos leads to higher PRs than the transfer of one embryo.

In contrast to the markedly reduced ongoing PR found in our study after ESET compared with DET in an unselected group of patients, the ongoing PR between eSET applied in a subset of good prognosis patients and DET applied in the remaining patients (33.0\% after SP-eSET versus $30.3 \%$ after SPDET) was comparable. This is in agreement with the observational studies described in literature, obtaining PRs of 34 versus 37\% (Tiitinen et al., 2003) and 40.3 versus $40.4 \%$ (Gerris et al., 2004), respectively, after applying ESET in a subgroup of good prognosis patients and DET in the remaining patients. The twin PR in the overall IVF programme when applying our standard embryo transfer policy was $12.9 \%$ in our study, which is comparable with the findings of other studies (7.5\%, (Tiitinen et al., 2003) and 13.5\%, (Gerris et al., 2004)).

The ongoing PR obtained with our standard transfer policy (eSET in selected patients) is higher than with a policy of transferring one embryo in all patients (31.5 versus $21.4 \%$ ). This is, at least for patients $<36$ years, confirmed by the data from Debrock and co-workers, who compared PRs before and after implementation of the new legislation on embryo transfer in Belgium (Debrock et al., 2005). Before implementation, one embryo was transferred just in case it was a good quality embryo.

After implementation, in patients $<36$ years, no selection based on embryo quality was made for eSET, which means that all patients $<36$ years received one embryo. The clinical PR decreased non-significantly from $41.0 \%$ before implementation to $35.1 \%$ after implementation.

Of all eligible patients in our study, $46 \%$ were randomized in the RCT part of the study, comparing favourably with the participation rate in other studies 


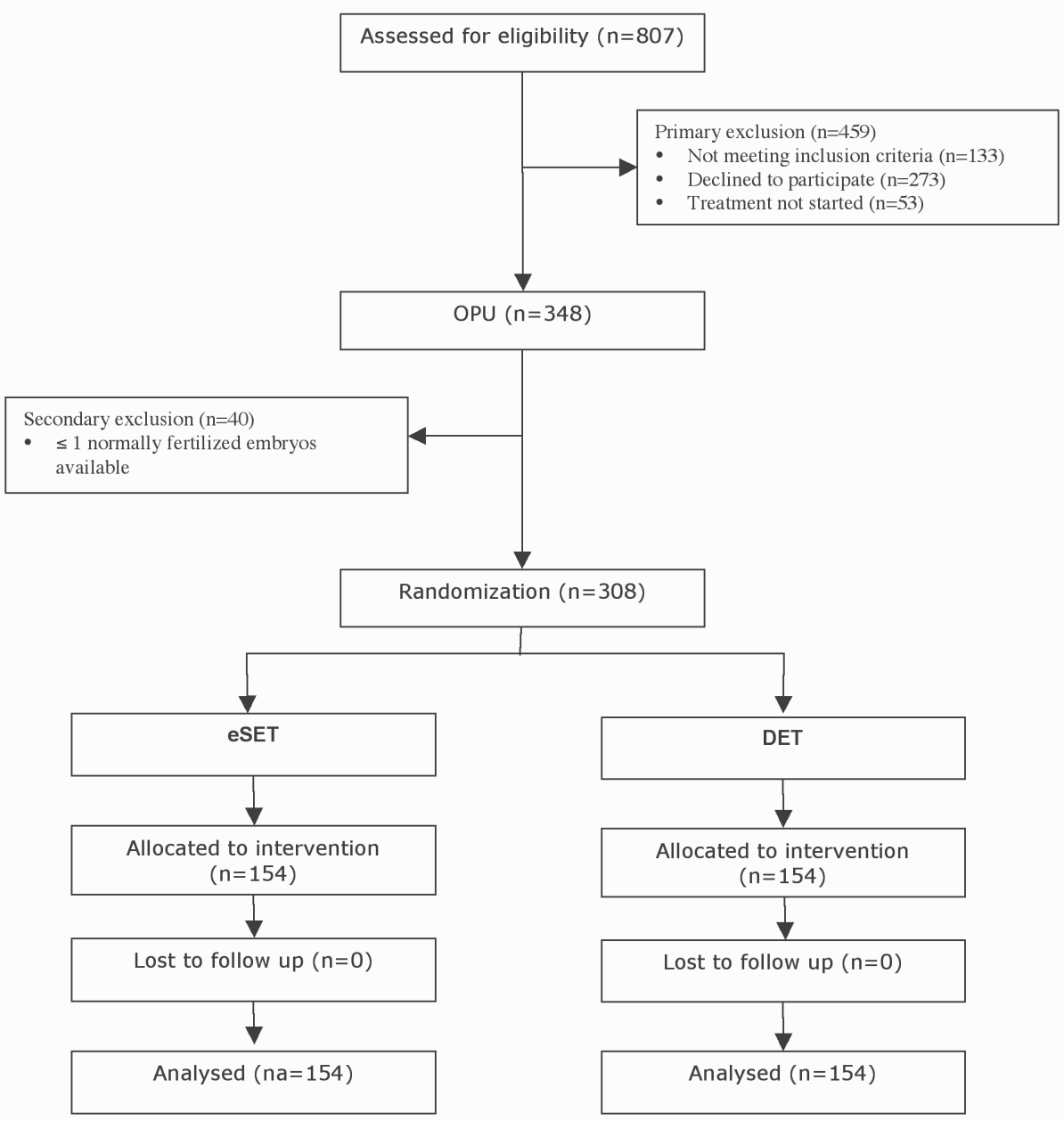

Figure 1: Flowchart of patient selection for the RCT part $f$ the study 


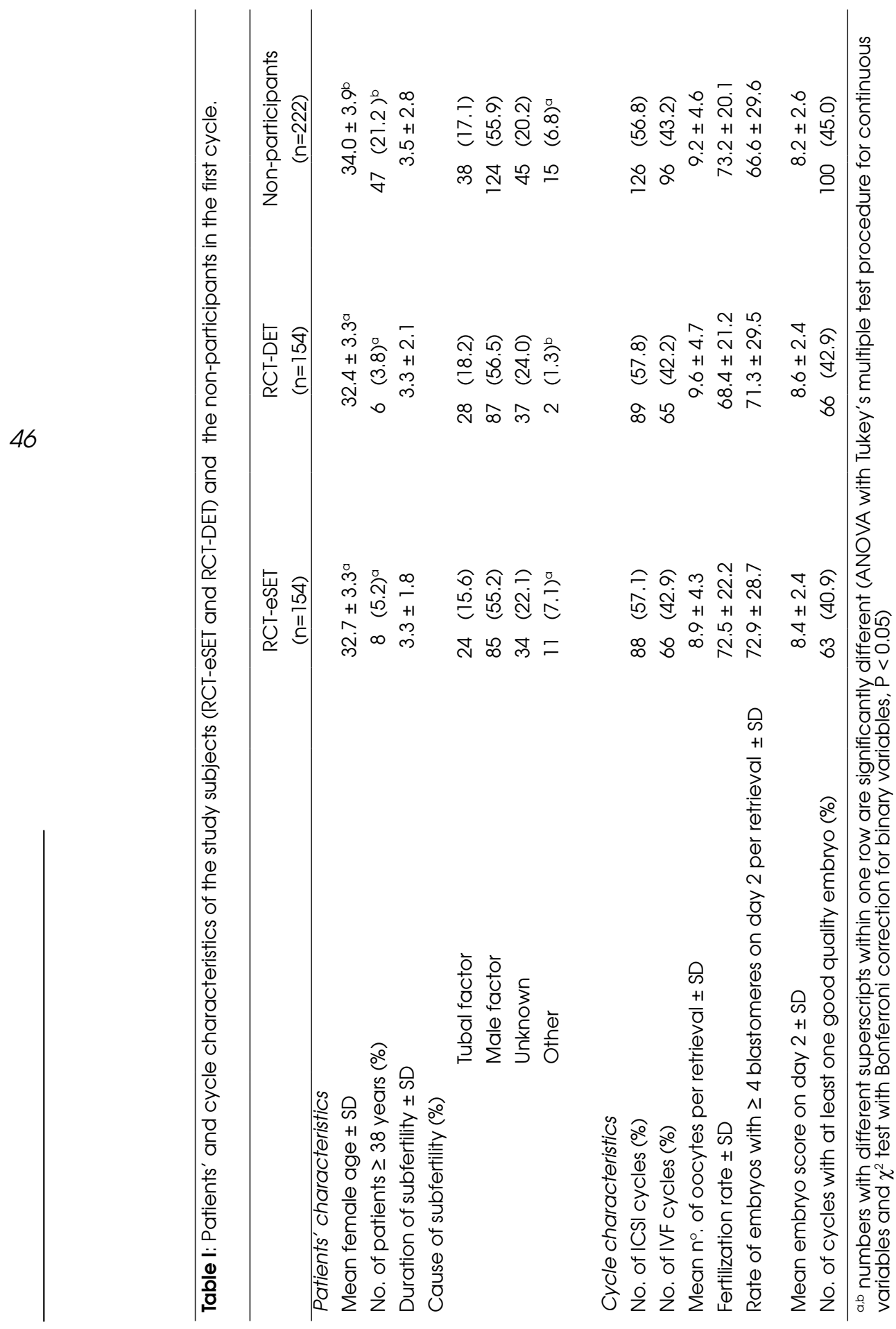



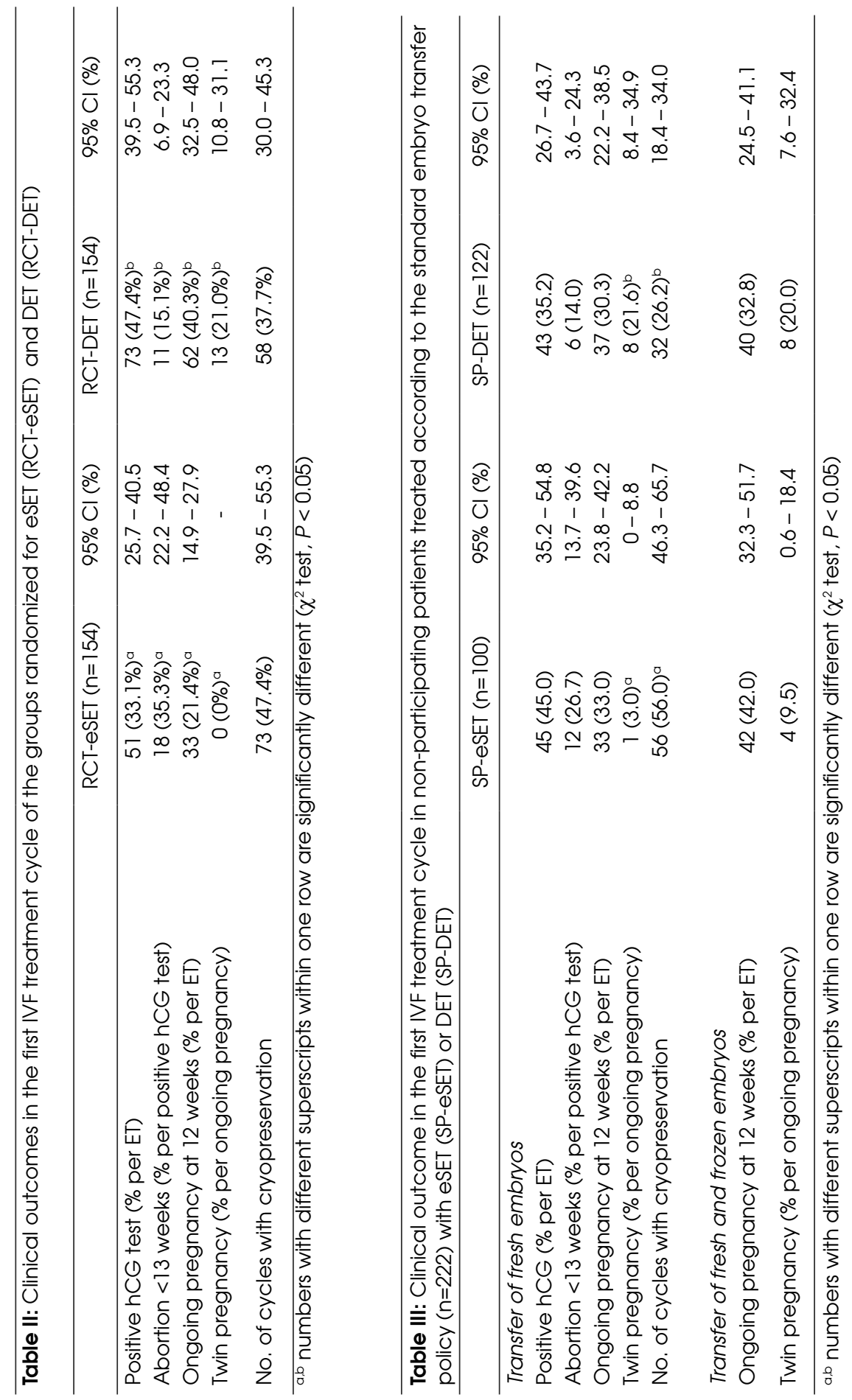
(11\%, (Martikainen et al., 2001); 25\%, (Thurin et al., 2004); 33\%, (Lukassen et al., 2005) and 39\%, (Gerris et al., 1999)). The higher participation rate in our study might explain the slightly lower PRs compared with the other RCTs described in literature. A high rate of participation will diminish selection bias. To evaluate selection bias in our study, the characteristics of the study population and the non-participants were compared. It was shown that the study population reflected the total IVF population with the exception of the percentage of patients $\geq 38$ years, which was $4.5 \%$ in the study population and $21.2 \%$ in the non-participants. This can be explained by the fact that patients $\geq 38$ years often considered their chance for pregnancy to be low and requested the standard embryo transfer policy, in which they would receive two embryos.

As our study shows, after the transfer of only a single embryo, more good quality embryos are left for cryopreservation. If the transfer of cryopreserved embryos (for practical reasons not a part of this RCT and therefore performed according to the standard transfer policy) was included in the results, the probability of a pregnancy after the first ovum pick-up increased. The overall PR was nevertheless still significantly lower in the eSET group as compared with the DET group (29.9\% versus $42.2 \%$, respectively). As from 17 eSET and 10 DET cycles a transfer of frozen/thawed embryos has not yet been performed, cumulative ongoing PRs will increase. The importance of cryopreserved embryos in ESET has already been stressed by other authors (Tiitinen et al., 2001; Thurin et al., 2004).

A remarkable finding in our study is the high abortion rate after eSET (35\% in the RCT-eSET group and $27 \%$ in the SP-eSET group). It is known that in the first weeks after implantation $15-20 \%$ of all pregnancies are lost, both in spontaneous pregnancies and in pregnancies conceived after assisted reproduction (Tummers et al., 2003; De Neubourg et al., 2004). Theoretically, the difference in abortion rate found between ESET and DET might be explained by vanishing twins in the DET group, which are continuing as a singleton pregnancy and therefore not recorded as an abortion. Since it has been shown that in pregnancies with two gestational sacs $\sim 30 \%$ result in a singleton pregnancy, only part of the difference in abortion rate between eSET and DET can be explained by vanishing twins (Landy and Keith, 1998). Winter et al. (2002) found that pregnancy loss before 6-7 weeks of gestation was related to poor embryo quality. However, in our study, the high abortion rate after eSET was found not only in the eSET group of the RCT part (in which $31 \%$ of the pregnancies developed after transfer of poor quality embryos) but also in the eSET group of our standard transfer policy group (in which eSET was performed with good quality embryos only). In addition, in the RCT-eSET group, 33\% of the pregnancies achieved after the transfer of a poor quality embryo and $38 \%$ of the pregnancies achieved after the transfer of a good quality embryo resulted in an abortion. This indicates that the abortion rate in our 
study was not related to poor embryo quality.

Whether ESET or DET is preferable depends not only on ongoing PRs and twin PRs, but also on several other factors, such as patients' preferences and the health care system in a particular country. Patients should be counselled thoroughly about PRs in the different transfer policies and about the risks associated with multiple pregnancies. Patients' attitudes towards eSET was shown to be positively adjusted in countries where new legislation regarding the embryo transfer was implemented, stating that eSET should be the routine procedure in at least the first cycle of young patients (Ombelet, 2004; Thurin et al., 2004). Finally, the extent of reimbursement of costs will influence the acceptance of eSET. Therefore, as an integral part of the present study the cost-effectiveness of eSET compared with DET was analysed (Fiddelers et al., 2005).

In conclusion, our study shows that applying eSET in the first cycle of an unselected group of patients will lead to a twin PR of $0 \%$. The price to be paid is a reduction of the ongoing PR to approximately half of that obtained after DET. The transfer of one embryo in a selected group of good prognosis patients leads to a less drastic reduction in PR but maintains a twin PR of $12.9 \%$.

\section{Acknowledgements}

We thank the following members of the IVF team in the Academic hospital Maastricht for their technical and clinical assistance:

Marijke Bras, Laurence Colautti, Edith Coonen, Janneke den Hartog, Kim van Kaam, Germaine Kengen, Marion Meijs, Nienke Muntjewerff, Annemiek Nap, Gisela Scheuermann, Inge Schreurs, Carla Schroen,MarieJosé Steijns, Roel Thijssen, Cécile Wolfs and Myriam Wolfs. 
CHAPTER 4

\section{Single versus double embryo transfer: cost-effectiveness analysis alongside a randomized clinical trial}

Audrey A.A. Fiddelers', Aafke P.A. van Montfoort ${ }^{2}$, Carmen D. Dirksen', John C.M. Dumoulin², Jolande A. Land ${ }^{2}$, Gerard A.J. Dunselman'2, J. Marij Janssen², Johan L. Severens',3, Johannes L.H. Evers²

'Department of Clinical Epidemiology and Medical Technology Assessment, ${ }^{2}$ Department of Obstetrics \& Gynaecology, Academic Hospital Maastricht, ${ }^{3}$ Department of Health Organisation, Policy, and Economics, University Maastricht, Maastricht, The Netherlands

Published in: Hum Reprod (2006), 21, 2090-2097. 


\section{Abstract}

BACKGROUND: Twin pregnancies after IVF are still frequent and are considered high-risk pregnancies leading to high costs. Transferring one embryo can reduce the twin pregnancy rate (PR). We compared costeffectiveness of one fresh cycle elective single embryo transfer (eSET) versus one fresh cycle double embryo transfer (DET) in an unselected patient population.

METHODS: Patients starting their first IVF cycle were randomized between ESET and DET. Societal costs per couple were determined empirically, from hormonal stimulation up to 42 weeks after embryo transfer. An incremental cost-effectiveness ratio (ICER) was calculated, representing additional costs per successful pregnancy.

RESULTS: Successful PRs were $20.8 \%$ for eSET and $39.6 \%$ for DET. Societal costs per couple were significantly lower after eSET ( $€ 7334)$ compared to DET ( $€ 10924$ ). The ICER of DET compared to eSET was $€ 19096$, meaning that each additional successful pregnancy in the DET group will cost $€ 19096$ extra.

CONCLUSIONS: One cycle eSET was less expensive, but also less effective compared to one cycle DET. It depends on society's willingness to pay for one extra successful pregnancy, whether one cycle DET is preferred from a cost-effectiveness point of view. 


\section{Introduction}

One of the most important complications of infertility treatments is the high percentage of multiple pregnancies. In 2001, of all IVF pregnancies in Europe, $24.0 \%$ were twin pregnancies (Andersen et al., 2005) compared with $1.2 \%$ twin pregnancies after natural conception (ESHRE Capri Workshop Group, 2000). Twin pregnancies are considered high-risk pregnancies for both mother and infants because of the relative high incidence in obstetric, perinatal and neonatal complications, which at the same time lead to high health-care costs. Up till now, five studies have been performed on the cost-effectiveness of elective single embryo transfer (eSET) versus double embryo transfer (DET) (Wolner-Hanssen and Rydhstroem, 1998; De Sutter et al., 2002; Gerris et al., 2004; Lukassen et al., 2005; Thurin Kjellberg et al., 2006).

One study from Sweden used a hypothetical take-home baby rate after SET and empirical pregnancy rates (PRs) after DET to compare costs per successful pregnancy (Wolner-Hanssen and Rydhstroem, 1998). Another study from Belgium (De Sutter et al., 2002) used a Markov model to estimate cost-effectiveness of eSET versus DET, in which pregnancy probabilities for ESET and DET were based on results from other studies, and costs were based on data from a local hospital. In a study by Gerris et al. (2004), also from Belgium, the cost-effectiveness of ESET and DET was compared by offering patients the choice of SET, in case one good quality embryo was available, and DET, irrespective of embryo quality. Furthermore, in a Dutch study by Lukassen et al. (2005), the cost-effectiveness of two cycles ESET was compared with one cycle DET after randomization, in case two embryos were available of which one was of good quality. Finally, in a Scandinavian study by Thurin Kjellberg et al. (2006), the cost-effectiveness of one cycle eSET and one cycle DET was compared, in case two good quality embryos were available in patients < 36 years of age before randomization for ESET or DET. In a proportion of the patients, three good quality embryos had to be available in patients $<35$ years of age.

What the former studies have in common is that eSET was performed in a sub-sample of good prognosis patients. A radical approach to reduce the twin PR would be to apply eSET in all patients, irrespective of their age and whether or not a good quality embryo is present. We have performed a randomized clinial trial (RCT), in which couples were randomized to having either eSET or DET in their first IVF treatment cycle, irrespective of age and embryo quality (Van Montfoort et al., 2006). Alongside the RCT, a costeffectiveness analysis was performed. The aim of this article is to compare the costs and cost-effectiveness of one fresh cycle eSET and one fresh cycle DET in an unselected sample of patients. 


\section{Materials and methods}

\section{Study design}

From January 2002 to December 2004, all couples who entered our IVF programme had their eligibility assessed for participation in this study (Van Montfoort et al., 2006); 308 couples who started their first IVF cycle were included in the study. Provided that at least two embryos (2PN) were available, couples were randomized between ESET and DET, irrespective of age and embryo quality. For a detailed overview of the patients and study design, see Van Montfoort et al. (2006).

\section{Cost analysis}

The cost analysis was performed from the societal perspective, according to the Dutch guidelines for cost calculations, and included health care costs and costs outside the health care sector (Oostenbrink et al., 2002; Oostenbrink et al., 2004). Health care costs consisted of hospital costs, and other health care costs related to the IVF procedure and resulting pregnancies. Hospital costs consisted of personnel costs, costs of material, costs of equipment, medication and overhead. Other health care costs included costs of visits to a General Practitioner (GP) or midwife care. Costs outside the healthcare system consisted of productivity costs and out of pocket costs for the couples (such as travel costs and over-thecounter-medication) associated with the IVF treatment cycle and resulting pregnancies. Costs were determined empirically for each couple entering the study from the start of the first IVF treatment cycle (i.e. hormonal stimulation) up to 42 weeks after embryo transfer (ET). For the subgroup of pregnant patients, this means that costs were determined up to 4 weeks after giving birth. For couples with a negative pregnancy test, we assumed no costs related to the first IVF treatment cycle were made after the first IVF cycle. Costs were calculated by multiplying volumes of use with unit prices. Subtotal costs were determined for hospital costs, other health care costs and costs outside health care made during the IVF treatment cycle, pregnancy as well as delivery and up to 4 weeks after delivery. All costs were determined for the year 2003.

\section{Volumes of use}

Cost diaries were used to determine volumes of use during the IVF treatment cycle, during pregnancy (from 13 weeks onwards, i.e. ongoing pregnancy), delivery and up to four weeks after delivery. In these diaries we asked for visits to a GP or midwife, for hospital visits, for hospital admissions, for over-the-counter medication, for the distance to several health care provisions, for absence of work, and other costs related to the IVF treatment cycle, pregnancy, or post-natal period (including delivery). The couples completed each cost diary prospectively for four successive weeks. The first cost diary was used to determine costs during the IVF treatment cycle, 
from hormonal stimulation ( 2 weeks before ET) until pregnancy test ( 2 weeks after ET), to reflect care directly related to IVF. For patients with a positive pregnancy test, no cost diary was administered to determine volumes of care until 13 weeks of pregnancy. Therefore, we assumed that they had one hospital visit, including an ultrasound at seven weeks of pregnancy. With respect to patients who miscarried before 13 weeks of pregnancy, we assumed they had another hospital visit including an ultrasound at the hospital (a total of two hospital visits and two ultrasounds). To determine volumes of use during pregnancy from 13 weeks onwards, patients with a singleton pregnancy were asked to complete two cost diaries for two randomly selected periods, whereas patients with a twin pregnancy completed three cost diaries for three randomly selected periods. Patients who were pregnant with twins were asked to complete three instead of two cost diaries, because only $20 \%$ of the pregnant patients in the DET strategy had a twin pregnancy (Van Montfoort et al., 2006). In that way, we still received sufficient information of medical consumption and other costs during pregnancy. To calculate the total costs per patient, the costs per period were added, assuming that the subgroup of patients ( $13-26$ patients per period, of whom two to five patients were pregnant with twins) was representative for the total group of pregnant patients. The last cost diary was used to determine volumes of use of delivery and after delivery, starting from delivery up to 4 weeks later.

\section{Unit prices}

Unit prices were obtained from the hospital financial department, by cost price calculation and by using guideline prices. All hospital costs during the IVF treatment cycle, except the costs of the laboratory phase were calculated using unit prices by the financial department of the University Hospital, Maastricht. These unit prices include the general overhead costs. Unit prices were linked to the volumes of use obtained from the cost diaries to determine the mean hospital costs per couple. The cost of the laboratory phase was determined by a detailed cost price calculation (Oostenbrink et al., 2004). First, the total costs for the year 2003 of all equipment used for IVF (depreciation, interest, and maintenance costs of invested capital) were determined. Furthermore, material costs related to IVF (such as needles and chemicals) and personnel costs were determined for the year 2003. The total direct costs were raised with $35 \%$ to include the general overhead of the hospital (Oostenbrink et al., 2004). To calculate the mean laboratory costs per IVF cycle per couple, total costs were divided by the number of ovum pick ups (OPUs) performed in 1 year.

For calculation of costs outside the hospital, guideline prices were used. Productivity losses were valued by using the friction cost method, which assumes that within a productivity process, everybody is replaceable. Productivity costs only appear during the friction period which is defined as the average period that a vacancy exists in a society (Oostenbrink et al., 2004; Brouwer and Koopmanschap, 2005). 
Cost-effectiveness analysis

A cost-effectiveness analysis was performed based on the incremental costs per extra successful pregnancy. A successful pregnancy was defined as a pregnancy resulting in at least one live-born child. The incremental cost effectiveness ratio (ICER) was calculated by using the following formula: (mean total costs DET - mean total costs eSET) / (proportion of successful pregnancies DET - proportion of successful pregnancies eSET).

\section{Sensitivity analyses}

Recently, an integrativestudy was performed in whichthe cost-effectiveness of several IVF treatment strategies in the Netherlands was determined, based on six IVF-related studies financed by the Dutch Organisation for Health Research and Development (ZonMw). For this, mean unit prices were determined, based on unit price information from all participating centres (ZonMw, 2005). These 'Dutch' unit prices were available for a limited number of cost units. First, a sensitivity analysis was performed in which these 'Dutch' unit prices were used instead of the Maastricht unit prices, to investigate the influence on the cost-effectiveness of eSET versus DET. Second, a sensitivity analysis was performed in which the costs of IVF medication were set on the lowest and highest values ( $€ 1046$ and $€ 1550$ ). Third, as costs from 5 to 12 weeks of pregnancy were based on an assumption, sensitivity analyses were performed in which a 'low-care' and 'high-care' assumption was made for estimation of the costs of that period. In the 'low-care' assumption, only half of the pregnant patients had one visit including an ultrasound and only half of the patients who miscarried had one visit including an ultrasound. Furthermore, in the 'highcare' assumption, all pregnant patients had two hospital visits including an ultrasound and one visit to a GP, and all patients who miscarried had two hospital visits including an ultrasound. Fourth, for the post-natal costs, cost data from Lukassen et al. (2004) were used, because in our study, we had information only on these costs from $29 \%$ of the pregnant patients (25\% of the twin pregnancies and $31 \%$ of the singleton pregnancies) who completed the last cost diary. However, in the study of Lukassen et al., only hospital costs were calculated. Therefore, only these costs were changed in the sensitivity analysis. Fifth, hospital admissions during the IVF treatment cycle were excluded from the cost calculation, because the difference in costs of hospital admissions during the IVF treatment cycle between eSET and DET could not be attributed to the ET policy. Sixth, the unit price of a hospital admission day was changed into guideline prices according to Oostenbrink et al. (2004) ( $€ 337$ and $€ 476$ per day for general hospital and university hospital, respectively). Finally, as the cost analysis was based on couples, in a secondary analysis, costs were determined for female patients only, i.e. those actually undergoing the first IVF treatment cycle, excluding male partners from the cost analysis. 
Statistical analysis

The statistical analysis was performed according to the intention-to-treat principle. A Missing Value Analysis (Statistics Package for Social Sciences, (SPSS)) was performed to estimate missing items in the cost diaries. To estimate 'missing' cost diaries, the overall groups' mean of eSET and DET was calculated, and resulting values were imputed (mean substitution). To quantify uncertainty around the cost-differences between eSET and DET, subtotal IVF costs, pregnancy costs and post-natal costs, and total societal costs for ESET and DET were bootstrapped. The bootstrap method estimates the sampling distribution of a statistic through a large number of simulations, based on sampling with replacement from the original data (Briggs et al., 1997). The results based on 1000 bootstrap replications of the costs for eSET and DET were used to calculate $95 \%$ uncertainty intervals (UI) around the cost-differences, based on the $2.5^{\text {th }}$ and $97.5^{\text {th }}$ percentiles. Also, bootstrap simulations were conducted to get insight in the uncertainty around the ICER (Efron and Tobshirani, 1993), yielding information about the joint distribution of the cost and effect differences. The results based on 1000 successive bootstrap replications were presented in a costeffectiveness plane. The horizontal axis of the cost-effectiveness plane shows the difference in effect between the groups and the vertical axis shows the difference in costs. The choice of a treatment strategy depends on the maximum amount of money that the society is prepared to pay for a gain in effectiveness, which is called the ceiling ratio. A ceiling ratio can vary from zero to infinity and can be represented by an imaginary straight line through the origin of the cost-effectiveness plane, starting with a slope of zero (representing a ceiling ratio of zero) and increasing the slope to 90 degrees (representing a ceiling ratio of infinity). For any given ceiling ratio, the proportion of bootstrap replications with an ICER equal to or lower than that particular ceiling ratio was determined. The probability that an intervention is cost-effective in relation to different values of the ceiling ratio was reflected in a cost-effectiveness acceptability curve (Van Hout et al., 1994). Also, a cost-effectiveness acceptability curve was derived using the results of the sensitivity analysis in which postnatal cost data of Lukassen et al. (2004) were used. All analyses were performed in SPSS 12.0 with the exception of the bootstrap analysis which was performed in Excel 2000 . 


\section{Results}

In table I, baseline socio-economic characteristics are given for both the groups. All characteristics were comparable for ESET and DET. Other patients' and cycle characteristics such as female age and fertilization rate were also comparable for both groups. For a detailed overview of the latter, we refer to our previous publication (Van Montfoort et al., 2006).

\section{Effectiveness}

Table II shows the clinical outcomes of one cycle eSET versus one cycle DET. After one cycle eSET and one cycle DET, 33.1 and $47.4 \%$ of the patients had a positive pregnancy test, respectively, and 21.4 and $40.3 \%$ achieved an ongoing pregnancy (more than 12 weeks of pregnancy). In both the groups, one late abortion (after 12 weeks of pregnancy) occurred, resulting in $20.8 \%$ successful pregnancies in the SET group and $39.6 \%$ in the DET group. Of the successful pregnancies, 0 and $19.6 \%$ were twins after one cycle eSET and DET, respectively (Van Montfoort et al., 2006).

\section{Cost-analysis}

Table III summarizes the costs divided in IVF treatment costs, costs of pregnancy, delivery and up to 4 weeks later, resulting in the total societal costs per randomized couple. Subtotal costs of the IVF treatment cycle were $€ 4431$ for one cycle eSET and $€ 4513$ for one cycle DET. The bootstrapped difference of these costs was not statistically significant (95\% UI: €-88 - €268). The cost difference was mainly caused by higher hospital costs after ET, because of costs of six hospital admissions in the DET group following OPU. In five of six patients, hospital admission occurred immediately after the OPU, before ET and randomisation. These admissions can therefore not be attributed to having DET. However, one patient was admitted because of ovarian hyperstimulation syndrome (OHSS) which occurred 9 days after ET. This could possibly be contributed to the fact that this patient was pregnant with twins, although a possible relationship between multiple pregnancy and OHSS has not been convincingly established in the literature. During pregnancy, all cost components were about twice as high in the DET group compared with the ESET group and the cost difference was mainly caused by differences in productivity costs and hospital costs. The bootstrapped difference of the costs during pregnancy was statistically significant (95\% UI: €928 - €2622). 
Table I: Socio-economic characteristics at baseline

\begin{tabular}{|c|c|c|c|c|}
\hline & \multicolumn{2}{|c|}{ SET $(n=154)$} & \multicolumn{2}{|c|}{$\operatorname{DET}(n=154)$} \\
\hline & Female & Male & Female & Male \\
\hline \multicolumn{5}{|l|}{ Marital state } \\
\hline Married & \multicolumn{2}{|c|}{$98(63.6)$} & \multicolumn{2}{|c|}{$97(63.0)$} \\
\hline Not married or living & \multicolumn{2}{|c|}{$2(1.3)$} & \multicolumn{2}{|c|}{$2(1.3)$} \\
\hline Living together & \multicolumn{2}{|c|}{$25(16.2)$} & \multicolumn{2}{|c|}{$25(16.2)$} \\
\hline Information missing & \multicolumn{2}{|c|}{$29(18.8)$} & \multicolumn{2}{|c|}{$30(19.5)$} \\
\hline \multicolumn{5}{|l|}{ Having a housekeeper } \\
\hline Yes & \multicolumn{2}{|c|}{$19(12.3)$} & \multicolumn{2}{|c|}{$17(11.0)$} \\
\hline No & \multicolumn{2}{|c|}{$108(70.1)$} & \multicolumn{2}{|c|}{$101(65.6)$} \\
\hline Information missing & \multicolumn{2}{|c|}{$27(17.5)$} & \multicolumn{2}{|c|}{$36(23.4)$} \\
\hline \multicolumn{5}{|l|}{ Education } \\
\hline Lower education & $19(12.3)$ & $22(14.3)$ & $20(13.0)$ & $18(11.7)$ \\
\hline Secondary ducation & $27(17.5)$ & $15(9.7)$ & $22(14.3)$ & $19(12.3)$ \\
\hline Higher education & $72(46.8)$ & $62(40.3)$ & $70(45.5)$ & $62(40.3)$ \\
\hline University & $7(4.5)$ & $10(6.5)$ & $12(7.8)$ & $12(7.8)$ \\
\hline Information missing & $29(18.8)$ & $45(29.2)$ & $30(19.5)$ & $43(27.9)$ \\
\hline \multicolumn{5}{|l|}{ Paid work } \\
\hline Yes & $110(71.4)$ & $105(68.2)$ & $107(69.5)$ & $112(72.7)$ \\
\hline No & $17(11.0)$ & $13(8.4)$ & $19(12.3)$ & $4(2.6)$ \\
\hline Information missing & $27(17.5)$ & $36(23.4)$ & $28(18.2)$ & $38(24.7)$ \\
\hline \multicolumn{5}{|l|}{ Voluntary work } \\
\hline Yes & $5(3.2)$ & $4(2.6)$ & $5(3.2)$ & $4(2.6)$ \\
\hline No & $122(79.2)$ & $114(74.0)$ & $121(78.6)$ & $112(72.7)$ \\
\hline Information missing & $27(17.5)$ & $36(23.4)$ & $28(18.2)$ & $38(24.7)$ \\
\hline \multicolumn{5}{|l|}{ Studying } \\
\hline Yes & $6(3.9)$ & $2(1.3)$ & $5(3.2)$ & $2(1.3)$ \\
\hline No & $121(82.5)$ & $116(75.3)$ & $121(78.6)$ & $114(74.0)$ \\
\hline Information missing & $27(17.5)$ & $36(23.4)$ & $28(18.2)$ & $38(24.7)$ \\
\hline \multicolumn{5}{|l|}{ Unemployed } \\
\hline Yes & $4(2.6)$ & $4(2.6)$ & $6(3.9)$ & $1(0.6)$ \\
\hline No & $123(79.9)$ & $114(74.0)$ & $120(77.9)$ & $115(74.7)$ \\
\hline Information missing & $27(17.5)$ & $36(23.4)$ & $28(18.2)$ & $38(24.7)$ \\
\hline
\end{tabular}




\begin{tabular}{llrrr}
\hline Table I: continued & & & \\
\hline $\begin{array}{l}\text { Work-disabled } \\
\text { Yes }\end{array}$ & $7(4.5)$ & $3(1.9)$ & $5(3.2)$ & $1(0.6)$ \\
$\quad$ No & $120(77.9)$ & $115(74.7)$ & $121(78.6)$ & $115(74.7)$ \\
Information missing & $27(17.5)$ & $36(23.4)$ & $28(18.2)$ & $38(24.7)$ \\
& & & & \\
Housekeeping & & & & \\
Yes & $53(34.4)$ & $13(8.4)$ & $52(33.8)$ & $10(6.5)$ \\
$\quad$ No & $74(48.1)$ & $105(68.2)$ & $74(48.1)$ & $106(68.8)$ \\
$\quad$ Information missing & $27(17.5)$ & $36(23.4)$ & $28(18.2)$ & $38(24.7)$ \\
& & & & \\
\hline
\end{tabular}

Table II: Clinical outcomes of one cycle eSET versus one cycle DET ${ }^{1}$

\begin{tabular}{lcc}
\hline & SET (n=154) & DET ( $n=154)$ \\
\hline Positive HCG test (\% per ET) & $51(33.1 \%)$ & $73(47.4 \%)$ \\
Abortion <13 weeks (\% per positive HCG test) & $18(35.3 \%)$ & $11(15.1 \%)$ \\
Ongoing pregnancies (\% per ET) & $33(21.4 \%)$ & $62(40.3 \%)$ \\
Successful pregnancies (\% per ET) & $32(20.8 \%)$ & $61(39.6 \%)$ \\
Twin pregnancies (\% per successful pregnancy) & $0(0 \%)$ & $12(19.6 \%)$ \\
Number of children born (\% per ET) & $32(20.8 \%)$ & $73(47.4 \%)$ \\
\hline
\end{tabular}

'Adapted from Van Montfoort et al. (2006). Outcomes expressed per ET as ESET and DET are the comparators in this study

From delivery until 4 weeks later, the higher costs of DET compared with ESET were mainly caused by differences in hospital costs and productivity costs. The bootstrapped difference of the costs after delivery was also statistically significant (95\% Ul: $€ 966-€ 2637$ ). The total societal costs per couple were $€ 7334$ for one cycle eSET compared to $€ 10924$ for one cycle DET, which was statistically significantly different (95\% UI: $€ 2060-€ 5290$ ). Most costs during the IVF treatment cycle, pregnancy and post-natal period were due to female patients $(97 \%$ of the total societal costs in both the groups). The male partners were mainly responsible for the 'leave of absence' costs during the IVF treatment cycle (70\%), pregnancy (27\%) and from delivery up to four weeks later (100\%). 
Cost-effectiveness analysis

The ICER of one cycle DET compared to one cycle eSET was $€ 19096$ for the base-case analysis (table IV). This means that the incremental costs are $€ 19096$ for one extra successful pregnancy if one cycle DET will be performed instead of one cycle eSET.

Figure 1 shows the cost-effectiveness plane for the base-case analysis, in which the results based on 1000 successive bootstrap replications are given, comparing one cycle DET to one cycle eSET. The incremental costeffectiveness acceptability curve of the base-case analysis in Figure 2 shows that until the ceiling ratio reaches $€ 15000$, the probability that one cycle DET is cost-effective is $0 \%$, as all bootstrapped ICERs were equal to or higher than $€ 15000$. When the ceiling ratio is between $€ 15000$ and $€ 32500$, the probability that one cycle DET is cost-effective increases and the probability that one cycle eSET is most cost-effective decreases. If the ceiling ratio is above $€ 32500$, the probability that one cycle DET is most cost-effective is $100 \%$, as all bootstrapped ICERs were equal to or lower than $€ 32500$.

\section{Sensitivity analyses}

Results of the sensitivity analyses are summarized in table IV. The ICERs of one cycle DET compared with one cycle eSET changed only marginally if several cost parameters were altered. Using post-natal hospital costs of Lukassen et al. (2004) in a sensitivity analysis had the largest effect on the ICER. Therefore, results from this analysis were bootstrapped, and a costeffectiveness acceptability curve was derived. Again, 100\% of the costeffectiveness pairs are located in the quadrant where one cycle DET is more effective but more costly compared with one cycle eSET (northeast quadrant; results not graphically presented). Figure 2 (Lukassen data) shows that the cost-effectiveness acceptability curve is slightly shifted to the left compared to the base-case curve in Figure 2 meaning that the results became slightly more favourable for DET. Until the ceiling ratio reaches $€ 12500$, the probability that one cycle eSET is most cost-effective is $100 \%$. When the ceiling ratio is between $€ 12500$ and $€ 25000$, the probability that one cycle eSET is most cost-effective decreases and the probability that one cycle DET is most cost-effective increases. If the ceiling ratio is above $€ 25000$, the probability that one cycle DET is most cost-effective is $100 \%$. As costs of pregnancy were based on a small number of pregnant couples, we also compared our hospital costs during pregnancy with the hospital costs found in the study of Lukassen et al. (2004). These costs ( $€ 238$ for eSET and $€ 595$ for DET) were highly comparable with the hospital costs we calculated in our study ( $€ 281$ for eSET and $€ 593$ for DET). Therefore, we decided not to perform a sensitivity analysis on these costs. 


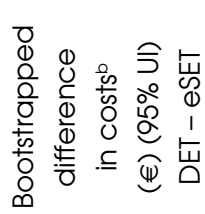

62

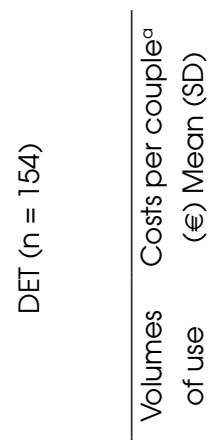

$\mathbb{1}$
0
0
$\frac{0}{0}$
0
$\frac{5}{5}$

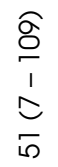

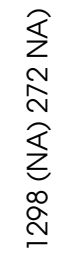

$=$

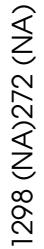

$=---\varangle 0$

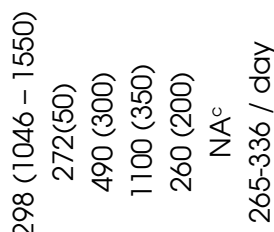

$\stackrel{\infty}{\mathrm{N}}$

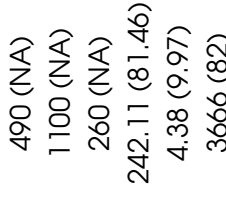

$\frac{N}{0} \longleftarrow$

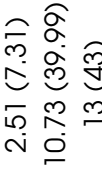

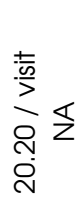

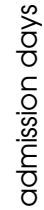

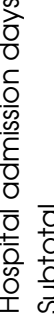

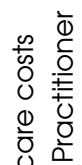

\begin{tabular}{l}
\multirow{2}{1}{} \\
1 \\
5 \\
7
\end{tabular}

焉

ิㅡ응

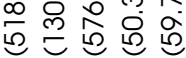

잉 웅

炙六 읏

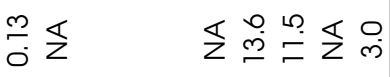

D.

. $\frac{0}{7}$

$\frac{0}{2}$

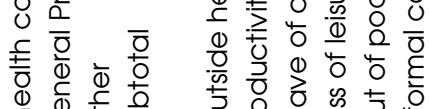

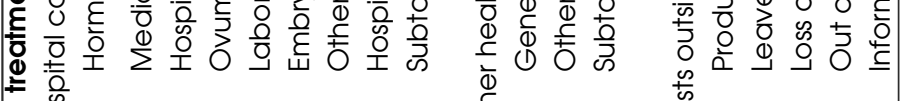

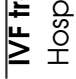

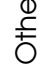

สิ่ง

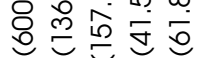

윷주

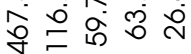

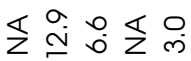

‡

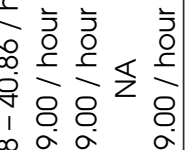

๙

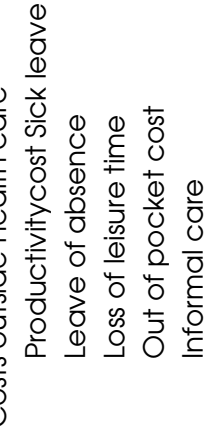




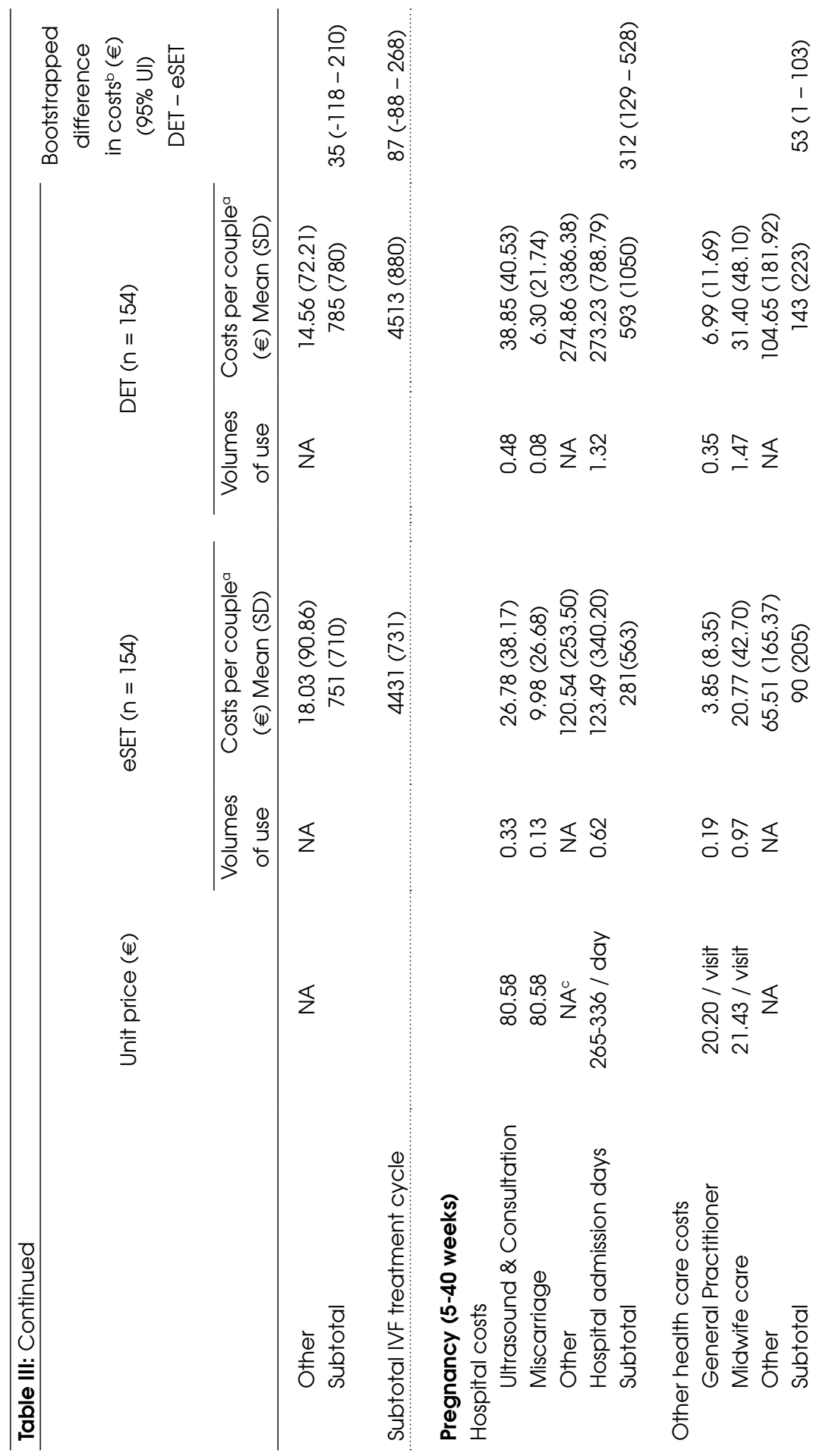




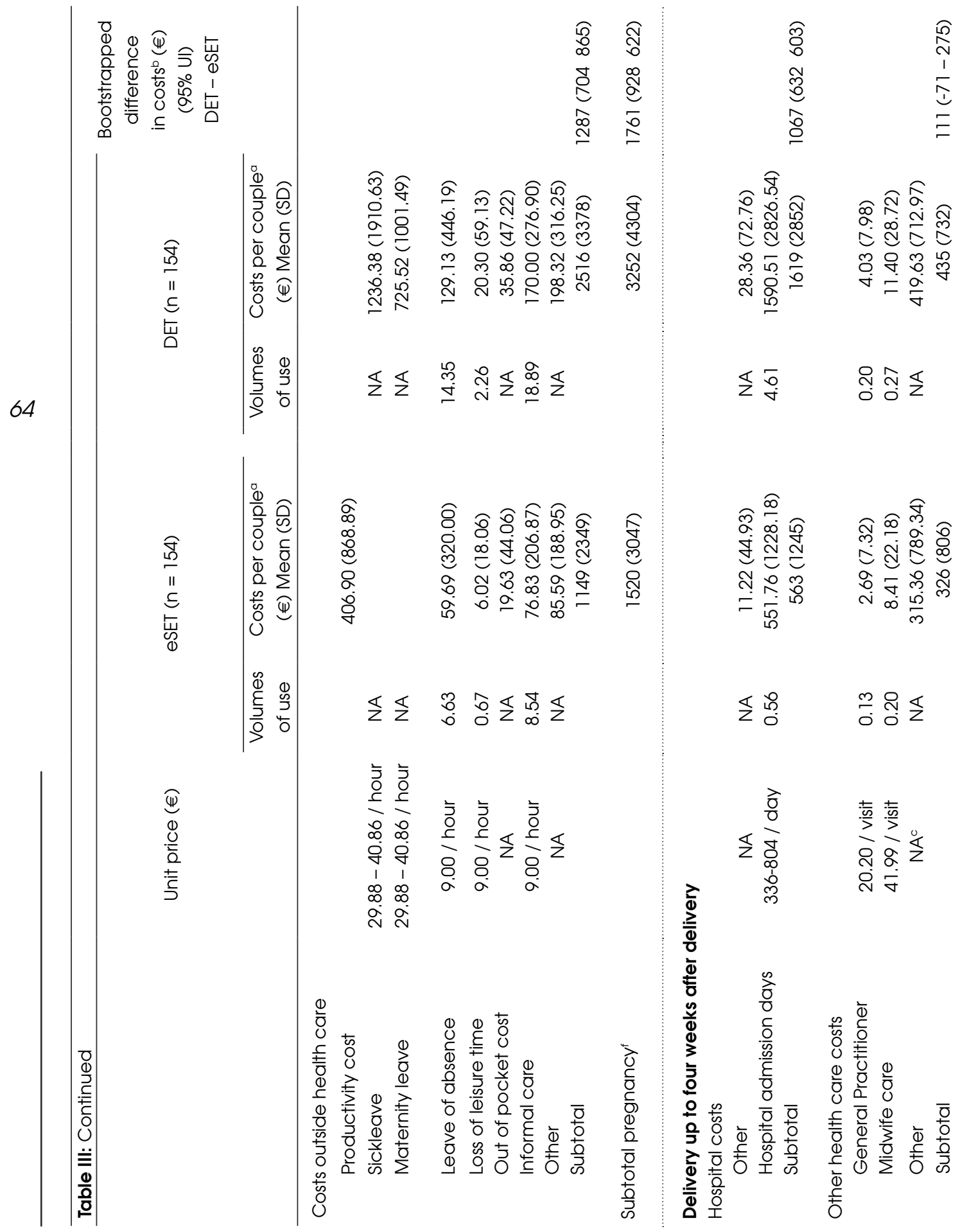




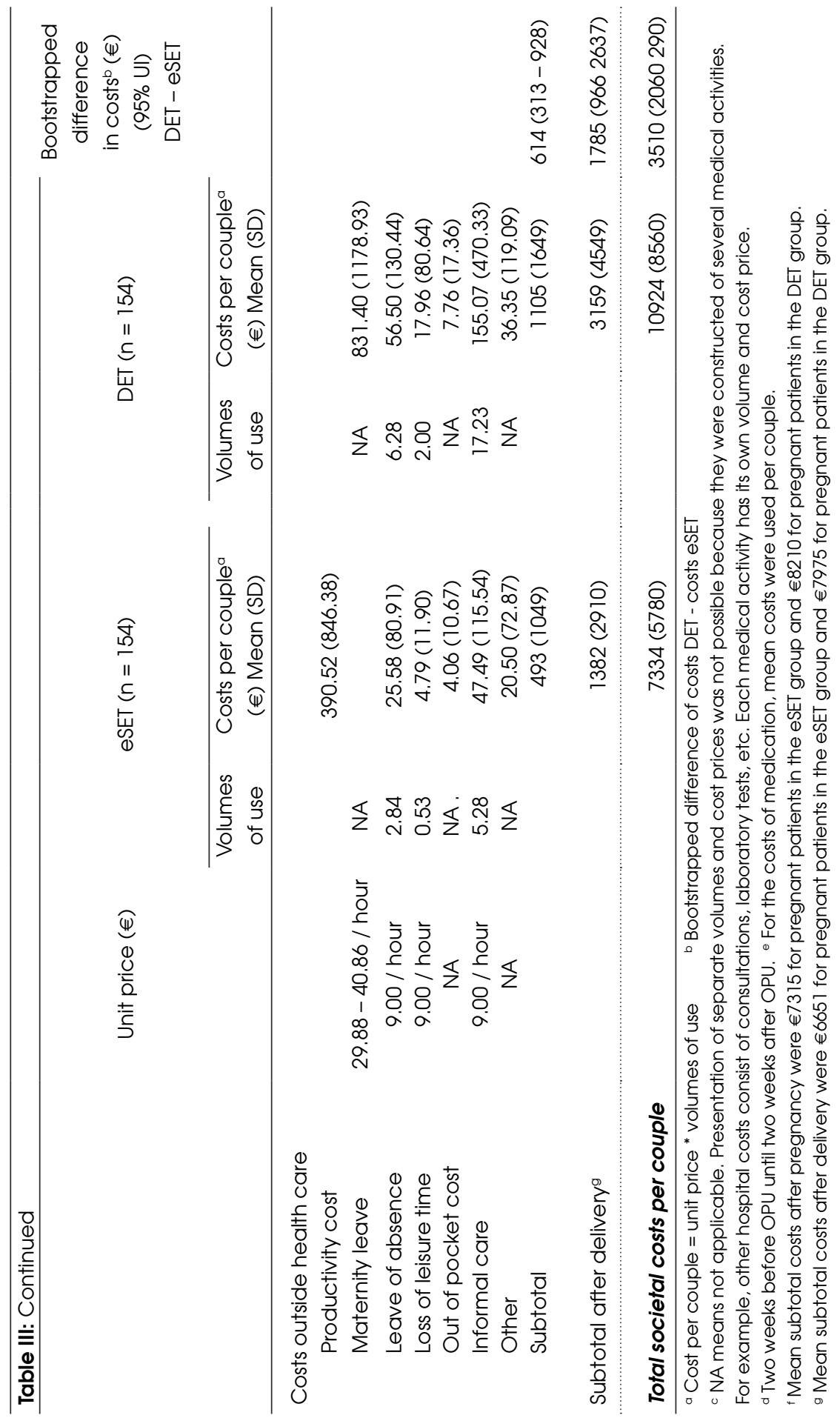




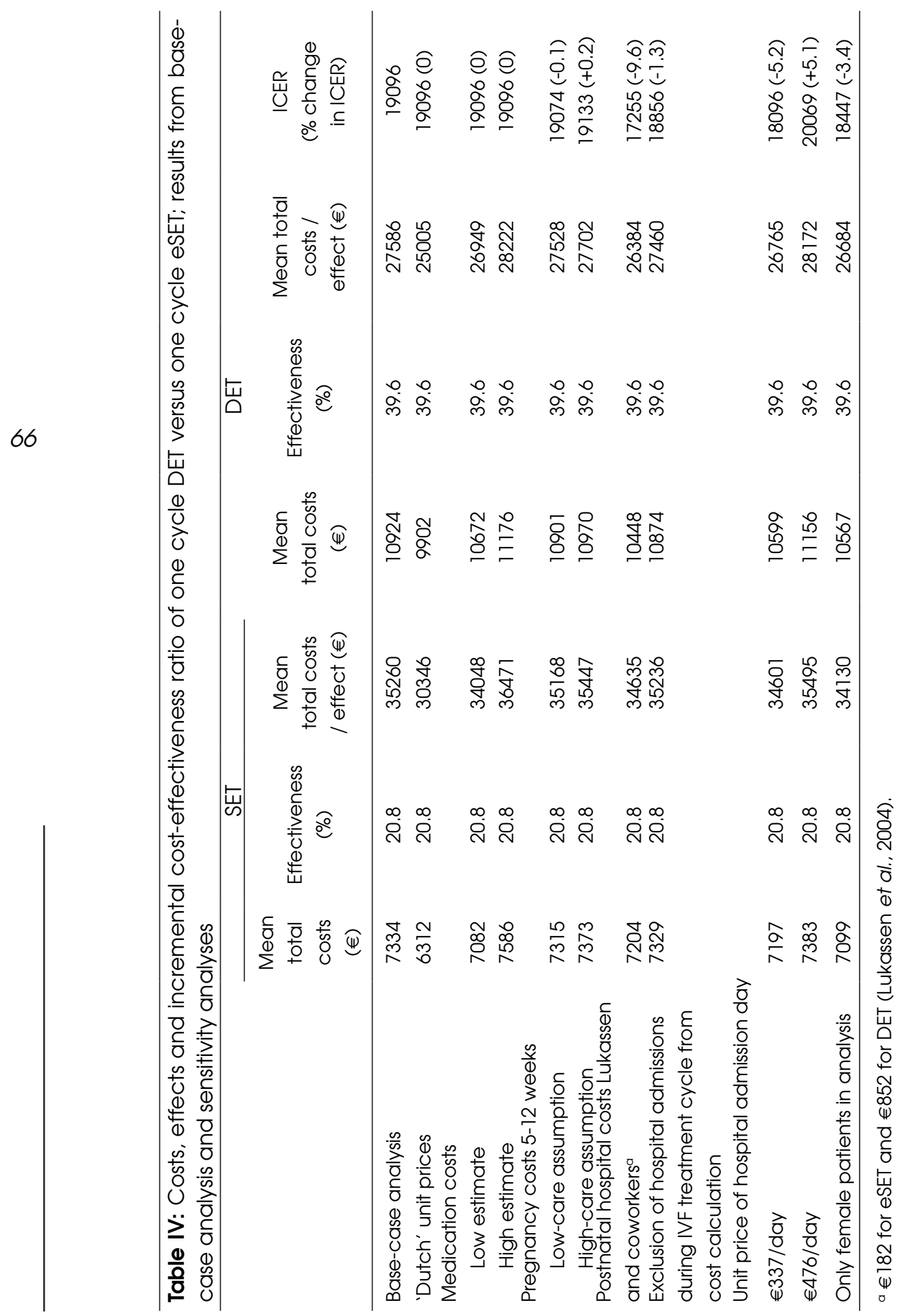




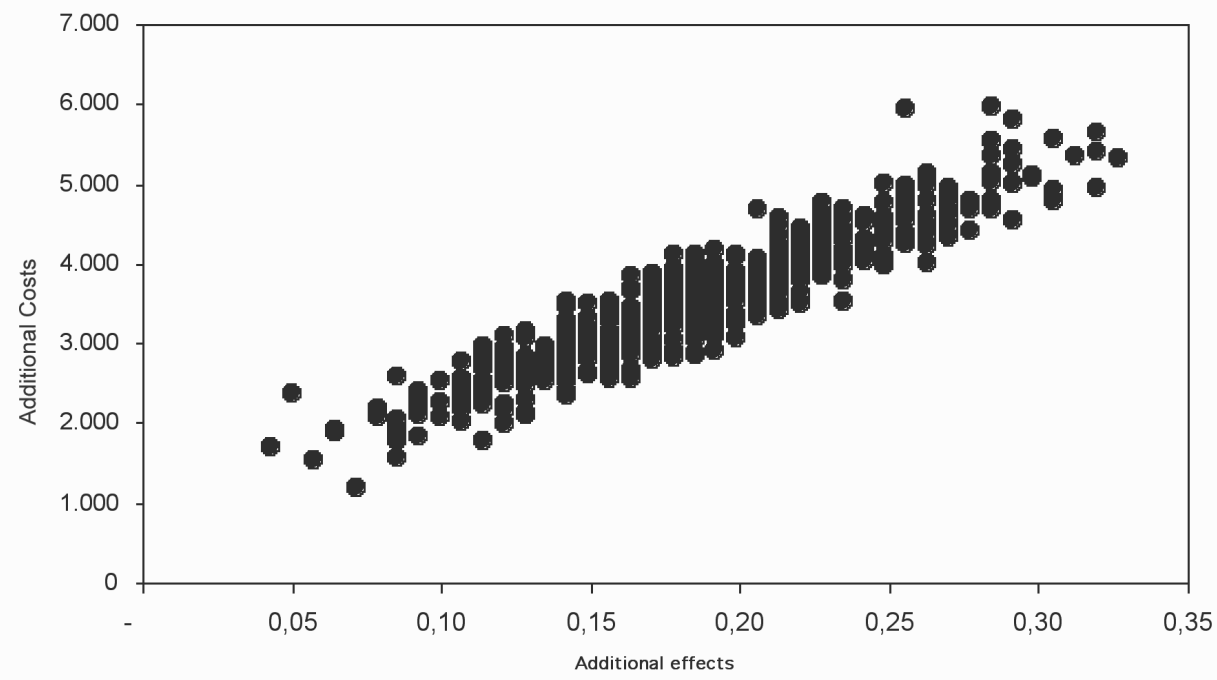

\section{Figure 1}

The cost-effectiveness plane is divided into four quadrants indicating four possible situations in relation to the additional costs and effects of one cycle DET compared to one cycle eSET (Briggs and Fenn, 1998). If the bootstrap replications are located in the northeast quadrant, DET will be more effective but also more costly compared to eSET. If they are in the southeast quadrant, DET is more effective and less costly compared to eSET, indicating dominance for DET. If they are in the southwest quadrant, DET is less costly, but also less effective compared with eSET. Finally, if they are in the northwest quadrant, DET is less effective and more costly compared to eSET, indicating inferiority for DET. The bootstrap analysis revealed that $100 \%$ of the cost-effectiveness pairs are located in the quadrant where one cycle DET is more effective but also more costly compared to one cycle eSET (northeast quadrant). Therefore, only thenortheast quadrant of the cost-effectiveness plane is shown. 


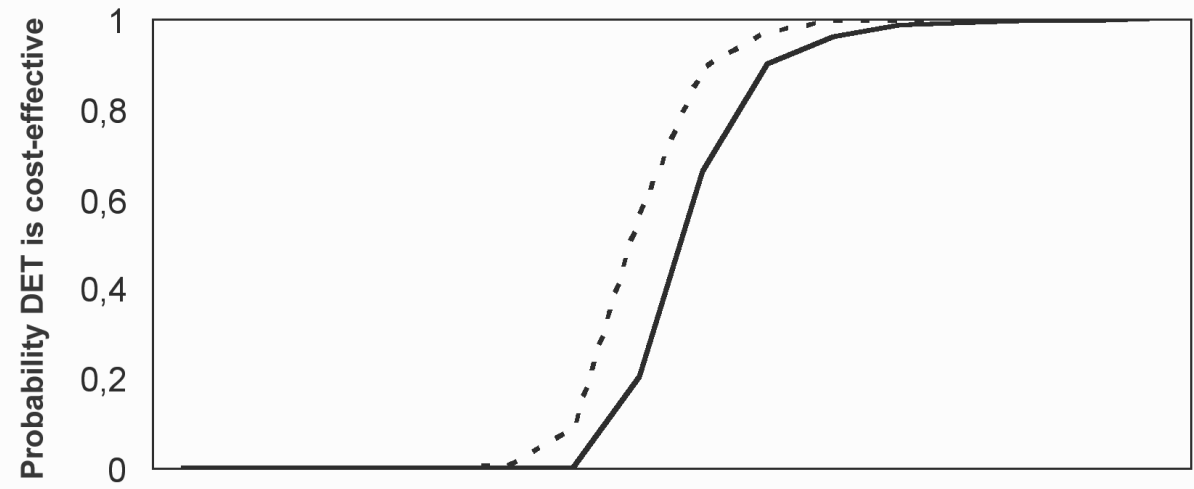

68

\section{Ceiling ratio}

- - - Lukassen-data —— Base case data

\section{Figure 2}

Cost-effectiveness acceptability curve for base-case analysis and for sensitivity analysis using postnatal costs of Lukassen et al. (2005) 


\section{Discussion}

This study is distinct from other studies, because a cost-effectiveness analysis was performed in an unselected group of patients receiving eSET or DET, based on the couple's perspective instead of only the female patient's perspective. Socio-economic characteristics were comparable for both the groups, indicating that the difference in costs between eSET and DET (such as productivity costs and out-of-pocket costs) was not affected by differences in socio-economic characteristics. The successful PR after eSET was approximately half of that obtained after DET (20.8\% after eSET versus $39.6 \%$ after DET). Total societal costs per couple were significantly lower after one cycle eSET ( $€ 7334)$ compared to one cycle DET ( $€ 10924)$.

The cost difference was mainly caused by the hospital costs after ET (40\% of the cost difference) and productivity costs (32\% of the cost difference). Female patients were mainly responsible for the costs in both the groups. The ICER of DET compared to that of eSET was $€ 19096$, indicating that the incremental costs are $€ 19096$ for one extra successful pregnancy if one cycle DET will be performed instead of one cycle eSET. Sensitivity analyses showed that our results were very robust for changing several cost parameters. Using post-natal hospital costs of Lukassen et al. (2004) caused a minor shift to the left in the cost-effectiveness acceptability curve, indicating that results became slightly more favourable for DET.

There are some limitations in the cost-effectiveness analysis that need to be addressed. First, all data with respect to the volumes of use were collected from cost diaries, because most patients dispersed to other hospitals in the south of the Netherlands during pregnancy. Because we relied on couple-reported data and made no use of official registrations, possibly under- or over-reporting may have occurred. However, it is expected that the bias resulting from it is probably equal for both the groups, so it will not influence our conclusions. Although several assumptions were made and some data were missing, our results proved to be reliable for changing several cost parameters. In addition, cost diaries have proven to be reliable (Goossens et al., 2000) and are commonly used in costeffectiveness analysis. Second, in our cost-effectiveness analysis only results of the first IVF cycle were compared.

Nowadays, there is increasing support to compare two consecutive cycles of eSET with one cycle of DET, to gain equal pregnancy probabilities. In our study, assuming that all couples without a successful pregnancy in the first ESET cycle would receive eSET for a second time, that the successful PR of a second cycle eSET would be exactly the same as in the first cycle and that the total costs would also be the same, $37.0 \%(32(20.8 \% * 154)+25$ $(20.8 \% * 122) / 154)$ of the patients would have a successful pregnancy after two cycles of eSET. The total costs would be $€ 12728$ (( $€ 7102 * 154)$ $+(€ 7102 * 122) / 154)$. On the basis of ICER, two cycles eSET would be 
dominated by one cycle DET, because costs are higher and the effect is lower compared with one cycle DET. Furthermore, in a comprehensive cost-effectiveness analysis, several DET and ESET strategies should be compared, evaluating the full IVF procedure. For this, a Markov model is currently being developed, reflecting the 'real-world' situation as accurate as possible, taking into account cancelled cycles, availability of only one embryo (compulsory SET), declining PRs in subsequent cycles, transfers of cryopreserved embryos and treatment dropouts. In this model several ESET and DET strategies based on continued cycles are compared to determine which one is preferable.

Former studies have shown that cost differences between eSET and DET are mainly caused by high costs because of complications during twin pregnancies and deliveries and by high neonatal costs for twins in the DET group (Wolner-Hanssen and Rydhstroem, 1998; De Sutter et al., 2002; Gerris et al., 2004; Lukassen et al., 2005; Thurin Kjellberg et al., 2006). In our study, the cost difference between ESET and DET was also because of twin pregnancies and more pregnancies occurring in the DET group. Contrary to most other cost-effectiveness studies (Wolner-Hanssen and Rydhstroem, 1998; De Sutter et al., 2002; Gerris et al., 2004; Lukassen et al., 2005) in our study, an ICER was calculated. Other studies have only calculated the mean costs per successful pregnancy (i.e. birth of at least one liveborn child) for each strategy separately. According to Drummond et al. (2005), regarding a full economic evaluation, two criteria have to be met. First, in the study, two or more alternatives have to be compared. Second, an economic evaluation must deal with the consequences (outcome) and the costs (investment) of the treatment alternatives being compared using an incremental approach. Therefore, it is not comprehensive to only determine the mean costs per effect for each strategy separately but the incremental cost-effectiveness should be reported as well. Moreover, as these studies used a selected patient group, results cannot directly be compared to those of the present study. Only in the study of Thurin Kjellberg et al. (2006) an ICER was calculated. Using a societal perspective, their ICER comparing DET with SET was €91702 per extra successful pregnancy, which is about 4.5 times higher than the ICER of our study. On the basis of ICER, Thurin and co-workers concluded that DET is not cost-effective.

The choice between offering couples one cycle eSET or one cycle DET depends on what society is prepared to pay for one extra live-born child. Currently, there is no universally accepted ceiling ratio for cost-effectiveness and to date (Eichler et al., 2004), most cost-effectiveness researchers only provide policymakers with cost-effectiveness acceptability curves, showing for a wide range of ceiling ratios, the probability that a particular health care commodity is cost-effective. Also within the field of IVF, there is currently no agreement on an appropriate ceiling ratio for one extra 
successful pregnancy. Therefore, based on the results of this study it cannot be concluded whether DET or eSET in the first cycle is more cost-effective. On the other hand, because DET seems to be current practice the ratio from our study indicates that substituting DET by ESET in an unselected patient group would lead to cost savings but effectiveness loss as well.

A reluctance to lose a proportion of successful pregnancies would consequently indicate acceptability of the ICER we found in this study (Severens et al., 2005). Nevertheless, in deciding on the preferred strategy, the long-term consequences of eSET and DET should also be considered. Inclusion of the long-term costs in the cost-effectiveness analysis, such as costs because of premature births, would probably result in a substantially higher ICER, making DET less attractive from a long-term economic point of view. However, a 'successful pregnancy' is considered an intermediate outcome measure. A commonly used outcome measure in economic evaluation is the number of quality adjusted life years (QALYs), combining the number of life years gained with the quality of that life. To obtain a balanced estimate of the long-term cost-effectiveness of eSET versus DET, both long-term costs and effects in terms of QALYs should be considered. For practical reasons, this has not been included in any study so far. Therefore, it is difficult to provide a fair estimate of the long-term costeffectiveness.

In conclusion, our study shows that in an unselected group of patients qualifying for IVF treatment, one cycle eSET is less expensive, but also less effective compared with one cycle DET. It depends on society's willingness to pay for one extra successful pregnancy, whether one cycle eSET or one cycle DET is preferred from a cost-effectiveness point of view. The Markov model currently in development by our research group should provide insight into the 'real-world' cost-effectiveness of several SET and DET strategies.

\section{Acknowledgements}

The authors wish to thank Fred Nieman (Dept. Clinical Epidemiology and medical Technology Assessment, University Hospital Maastricht) for his statistical assistance. 
CHAPTER 5

\section{eSET irrespective of the availability of a good quality embryo in the first cycle only is not effective in reducing overall twin pregnancy rates}

Aafke P.A. van Montfoort' ${ }^{\text {, Audrey A.A. Fiddelers }}{ }^{2}$, Jolande A. Land ${ }^{14}$, Carmen D. Dirksen², Johan L. Severens' ${ }^{2}$, Joep P.M. Geraedts ${ }^{3}$, Johannes L.H. Evers', John C.M. Dumoulin'

Research Institute Growth \& Development (GROW), 'Department of Obstetrics \& Gynaecology, ${ }^{2}$ Department of Clinical Epidemiology and Medical Technology Assessment, and ${ }^{3}$ Department of Clinical Genetics,

Academic Hospital Maastricht, Maastricht, the Netherlands ${ }^{4}$ Present address: Department of Obstetrics and Gynaecology, University Medical Center Groningen, Groningen, The Netherlands 


\section{Abstract}

INTRODUCTION: In several clinics elective single embryo transfer (eSET) is applied in a selected group of patients based on age and the availability of a good quality embryo. Whether or not eSET can be applied irrespective of the presence of a good quality embryo in the first cycle, to further reduce the twin pregnancy rate (PR), remains to be elucidated.

METHODS: In patients < 38 years two transfer strategies were compared, which differed in the first cycle only: group $A(n=141)$ received eSET irrespective of the availability of a good quality embryo, and group $B$ $(n=174)$ received eSET when a good quality embryo was available while otherwise they received DET (referred to as eSET/DET transfer policy). In any subsequent cycle, in both groups the eSET/DET transfer policy was applied.

RESULTS: After completion of their IVF treatment (including a maximum of three fresh cycles and the transfer of frozen-thawed embryos), comparable cumulative live birth rates (62.4\% in group A and $62.6 \%$ in group B) and twin pregnancies rates (10.1\% vs $13.4 \%$ ) were found. However, patients in group A required significantly more fresh ( $2.0 \mathrm{vs} 1.8)$ and frozen cycles $(0.8$ vs 0.5).

CONCLUSION: The transfer of one embryo in the first cycle only, in all patients $<38$ years, is not an effective transfer policy for reducing the overall twin PR. 


\section{Introduction}

In several randomized studies, elective Single Embryo Transfer (eSET, i.e. the transfer of one embryo in case one could also transfer two or more embryos) has proven its merits for reducing the twin pregnancy rate (PR), while obtaining acceptable overall PRs in selected patients (see Bergh (2005) for review on eSET). Based on these studies in several clinics a transfer policy was introduced in which relatively young patients with at least one or two good quality embryos received ESET and the remaining patients received double embryo transfer (DET) (referred to as eSET/DET transfer policy). This policy was applied in most studies in the first and second IVF cycle. Recently it was shown that this policy also led to acceptable results in the third IVF cycle (Van Montfoort et al., 2005).

To further increase the implementation of ESET a new strategy is applied in Belgium. Since July 2003 the legislation prescribes the reimbursement of six cycles in a life-time per patient under the condition that in the first cycle of patients $<36$ years, only one embryo is transferred, irrespective of the availability of a good quality embryo. In the subsequent cycles and in the cycles in older patients, transfer of a maximum of two or three embryos is allowed (Ombelet et al., 2005). Studies comparing the IVF results before and after the implementation of the new legislation in Belgium showed no difference in the overall ongoing PR per cycle, and a reduction in the overall twin PR, although studying two different strategies in two subsequent time-periods has its limitations (Debrock et al., 2005; Gordts et al., 2005). Besides, in these studies only outcome per cycle in two time periods was compared instead of cumulative outcome per patient.

Our group published a study in which the transfer strategy was comparable to the transfer strategy in the first cycle in Belgium, although in our study no age limit was set (Van Montfoort et al., 2006). In this randomized controlled trial (RCT) ESET was applied in the first cycle of an unselected patient population, i.e. irrespective of the availability of a good quality embryo and age. This strategy however led to a significant reduction in the ongoing PR as compared to DET or an eSET/DET policy in the first cycle (Van Montfoort et al., 2006). Whether or not this decrease in PR and the reduction in twin PR have their influence on the total IVF outcome per patient after several cycles of an ESET/DET policy remains to be elucidated.

The aim of the present study was therefore to compare two treatment strategies, performed in the same time-period, which are similar for the second and third cycle (i.e. eSET/DET policy) but differ in the first cycle; one group receiving eSET irrespective of the availability of a good quality embryo and another group receiving an eSET/DET transfer policy. The two groups, consisting of patients $<38$ years, were compared with respect to the total number of cycles, the live birth rate and the twin PR after a maximum of three cycles. 


\section{Methods}

\section{Patients}

In the IVF clinic of the academic hospital Maastricht eSET is routinely performed in patients $<38$ years when at least one good quality embryo is present (see below). If one of the criteria is not fulfilled, DET is performed. This transfer policy is referred to as the eSET/DET transfer policy and is applied in all treatment cycles of a patient. From January 2002 until December 2004, a RCT was performed in which consenting patients were randomly allocated to either eSET or DET in their first IVF or ICSI cycle, irrespective of the availability of a good quality embryo and female age. The only exclusion criteria were preimplantation genetic diagnosis (PGD), request of SET for medical reasons, language barrier (patients could not be well informed) and less than two 2PN embryos available. Patients fulfilling these criteria but declining to participate received our eSET/DET transfer policy (Van Montfoort et al., 2006). This study was approved by the Institutional Ethical Board of the academic hospital of Maastricht.

In the present study the results of two transfer strategies were compared, which only differed in the first cycle: group A receiving eSET irrespective of the availability of a good quality embryo (i.e. patients randomized for ESET in our RCT) and group B receiving the eSET/DET transfer policy (i.e. patients declining to participate in our RCT). As the age limit for the eSET/ DET policy in group $B$ was 38 years, also in group $A$ only patients $<38$ years were included in the analysis. In the second and third cycle of all patients (a maximum of three cycles is reimbursed in the Netherlands) the eSET/ DET transfer policy was applied. In case supernumerary embryos were cryopreserved, and no pregnancy was established, the cryopreserved embryos were transferred before a new ovum pick-up was performed. The transfer of these cryopreserved embryos was performed in all cycles of both groups according to the eSET/DET policy.

Ovarian stimulation, embryo culture and quality assessment

Ovarian stimulation, IVF, ICSI and embryo culture procedures have been described in detail earlier (Van Montfoort et al., 2006). For each embryo originating from a normally fertilized oocyte, an embryo score was calculated on the basis of morphological grade ( 1 to 4 , with grade 4 being the best grade), number of blastomeres and presence or absence of multinucleated blastomeres (MNBs) (Van Montfoort et al., 2005). This score ranged on day 2 from 1-16.5 with a higher score correlating to a better embryo quality. Embryos that had reached the 4- or 5-cell stage on day two, or the 8-cell stage on day three, in combination with having the best morphological grade (regular, even sized blastomeres with less than $20 \%$ fragmentation) and an absence of MNBs were classified as good quality embryos (score $\geq 13.25$ on day 2 or $\geq 23.25$ on day 3 ) (Van Montfoort et 
al., 2005). Embryos were transferred on day 2 after ovum pick-up or in a minority of cases, for reasons of convenience, on day 3. In all cases, including those in the RCT study, embryos with the highest embryo score were transferred.

Cryopreservation of supernumerary embryos was performed on the morning of the third day after ovum pick-up depending on their embryo score. The vast majority of cryopreserved embryos had reached the 7- to 8-cell stage in combination with morphological grade 3 or 4 .

\section{Statistical analysis}

Each patient was followed until a live birth either after the transfer of fresh or frozen/thawed embryos was registered, the patient had completed an IVF or ICSI treatment with a maximum of three ovum pick-ups (and any frozen-thawed embryo transfers) without success or the patient dropped out for medical or personal reasons. In this study a cycle is defined as a stimulation cycle resulting in an ovum pick-up. The cycles not resulting in an ovum pick-up are left out of consideration. A miscarriage was defined as a proven loss of the pregnancy and/or no fetal heart beat on ultrasound examination at or before 12 weeks gestation in a patient with an initially positive hCG test at two weeks after embryo transfer (detection limit 50 IU/ I in urine). An independent sample t-test was used to compare continuous variables, and the $\chi^{2}$ test was used for binary variables. A $P$-value $<0.05$ was considered significant.

To analyse whether or not differences in drop-out rate between the two groups might interfere with the outcome (i.e. cumulative live birth rates) a life-table analysis was performed (Cooke et al., 1981). As patients dropping out for medical reasons (e.g. poor response on ovarian stimulation) are assumed to have a lower live birth rate irrespective of the treatment strategy, these patients were excluded (Cooke et al., 1981; Daya, 2005). Especially patients dropping out for personal reasons (e.g. relocation to other part of country or the burden of treatment) or because of a spontaneous pregnancy can influence cumulative outcome, as these patients are likely to have similar live birth rates as compared to patients not lost to follow up (Daya, 2005). As the live birth rate of the patients lost to follow up is unknown, life table analysis was performed with two different assumptions; the live birth rate in patients dropping out to be $0 \%$ and to be similar to that in patients remaining in the study. 


\section{Results}

Group A consisted of 146 patients and group B of 175 patients. Those who had not completed their treatment at the time of analysis (23 months after the first ovum pick-up of the last patient included) were excluded from the analysis (5 and 1 patients respectively). Therefore the analysis was performed on 141 patients in group A and 174 patients in group B.

Both groups were comparable regarding patient and first cycle characteristics (Table I). Table II presents the clinical outcomes of the first cycle.. In group A all patients had ESET, and in group B ESET was performed in 100 patients ( $57.5 \%$ ). The transfer of two embryos in $42.5 \%$ of the patients in group B led to a significantly higher live birth and twin PR as compared with group A (33.9\% vs $21.3 \%$ and $13.3 \%$ vs $0 \%$, respectively). After the transfer of cryopreserved embryos in patients not achieving a pregnancy in the fresh cycle, live birth and twin PRs were comparable $32.6 \%$ and $8.5 \%$ in group $A$ and $39.7 \%$ and $14.3 \%$ in group B, Table II). In table III and IV the results of the first fresh cycles of group $A$ and $B$ are classified according to the transfer of good or moderate quality embryos. Although in group $A$ in both cases one embryo was transferred, it did not result in a significant difference in clinical outcome (Table III). In group B one good quality embryo or two moderate quality embryos were transferred (Table IV). With the exception of the twin PR, no significant difference was found between ESET and DET in group B. Also no significant difference was found in clinical outcome between eSET with a good quality embryo in group A (Table III) and B (Table IV). 
Table I : Patients' and first cycle characteristics

\begin{tabular}{lcc}
\hline & $\begin{array}{c}\text { Group A } \\
(\mathrm{n}=141)\end{array}$ & $\begin{array}{c}\text { Group B } \\
(\mathrm{n}=174)\end{array}$ \\
\hline Patients' characteristics & $32.3 \pm 0.3$ & $32.8 \pm 0.3$ \\
Mean female age \pm SE (year) & $3.3 \pm 0.1$ & $3.4 \pm 0.2$ \\
Duration of subfertility \pm SE (year) & $107(75.9)$ & $115 \quad(66.1)$ \\
Primary subfertility (\%) & & $27(15.6)$ \\
Cause of subfertility (\%) & $81(57.4)$ & $107(61.5)$ \\
$\quad$ Tubal factor & $6(4.3)$ & $3 \quad(1.7)$ \\
$\quad$ Male factor & $31(22.0)$ & $37(21.3)$ \\
$\quad$ endometriosis & $1(0.7)$ &
\end{tabular}

First cycle characteristics

ICSI cycles (\%)

$83(58.9) \quad 105(60.3)$

IVF cycles (\%)

$58(41.1)$

$69(39.7)$

Mean $n^{\circ}$. of oocytes per retrieval $\pm \mathrm{SE}$

$8.9 \pm 0.4$

$9.0 \pm 0.4$

Fertilization rate \pm SE

$73.4 \pm 1.6$

$73.6 \pm 1.6$

Rate of embryos with $\geq 4$ blastomeres on day 2

$72.8 \pm 2.5$

$66.0 \pm 2.3$

per retrieval $\pm \mathrm{SE}$

Mean embryo score on day $2 \pm$ SE

$8.4 \pm 0.2 \quad 8.3 \pm 0.2$

Mean no of transferred embryos \pm SE

$1.00 \pm 0.0^{a} \quad 1.56 \pm 0.5^{b}$

Mean no of frozen embryos per cycle \pm SE

$1.60 \pm 0.2 \quad 1.29 \pm 0.2$

No. of cycles with $\geq 1$ good quality embryo (\%)

$59(40.4)$

$72(41.4)$

\footnotetext{
a.b Numbers with different superscripts within one row are significantly different (independent sample $t$-test for continuous variables and $\chi^{2}$ test for binary variables, $P<0.05$ )
} 
Table II: Clinical outcome of the first cycle for group A and B

\begin{tabular}{|c|c|c|c|c|}
\hline \multirow[b]{2}{*}{ Fresh } & $\begin{array}{l}\text { Group A, } \\
n=141(\%)\end{array}$ & \multirow[t]{2}{*}{$\begin{array}{c}95 \% \mathrm{Cl} \\
(\%)\end{array}$} & $\begin{array}{l}\text { Group B, } \\
n=174(\%)\end{array}$ & \multirow[t]{2}{*}{$95 \% \mathrm{Cl}(\%)$} \\
\hline & & & & \\
\hline OPU & 141 & & 174 & \\
\hline eSET & $141(100)^{a}$ & $0-100$ & $100(57.5)^{a}$ & $50.2-64.8$ \\
\hline Positive hCG test & $46(32.6)$ & $24.9-40.3$ & $75(43.1)$ & $35.7-50.5$ \\
\hline Miscarriage & $15(32.6)$ & $19.1-46.1$ & $15(20.0)$ & 10.9-29. 1 \\
\hline Pregnancy (12 wks) & $31(22.0)^{a}$ & $15.2-28.8$ & $60(34.5)^{a}$ & $27.4-41.6$ \\
\hline Twin pregnancy & $0(0)^{a}$ & - & $8(13.3)^{a}$ & $4.7-21.9$ \\
\hline Live birth & $30(21.3)^{a}$ & $14.5-28.1$ & $59(33.9)^{a}$ & $26.9-40.9$ \\
\hline $\begin{array}{l}\text { Cycles with cryo- } \\
\text { preservation }\end{array}$ & $70(49.6)$ & $41.3-57.9$ & $73(42.0)$ & $34.7-49.3$ \\
\hline \multicolumn{5}{|l|}{ Cryopreserved } \\
\hline Thaw cycles & $\begin{array}{ll}59 & 0.4 / \\
& \text { patient }^{a}\end{array}$ & $0.3-0.5$ & $\begin{array}{ll}42 & 0.2 / \\
& \text { patient }\end{array}$ & $0.2-0.3$ \\
\hline Pregnancy (12 wks) & $16(27.1)$ & $15.8-38.4$ & $10(24.4)$ & $11.4-37.4$ \\
\hline Twin pregnancy & $4(25.0)$ & $3.8-46.2$ & $2(20.0)$ & $0-44.8$ \\
\hline
\end{tabular}

Cumulative

Pregnancy (12 wks)

$47(33.3)$

$25.5-41.1$

$70(40.2)$

$32.9-47.5$

Twin pregnancy

$4(8.5)$

0.5-16.5

10 (14.3)

6. $1-22.5$

Live birth

46 (32.6) 24.9-40.3

69 (39.7)

32.4-47.0

a Numbers with similar superscripts within one row are significantly different ( $\chi^{2}$ test, $P<0.05$ )

Table III: Clinical outcome of patients in group $A(n=141)$ classified into receiving a good quality embryo or not in the first cycle

\begin{tabular}{|c|c|c|c|c|}
\hline & $\begin{array}{c}\text { Good quality } \\
\text { embryo } \\
\text { available } \\
n=55(\%)\end{array}$ & $\begin{array}{c}95 \% \mathrm{Cl} \\
(\%)\end{array}$ & $\begin{array}{c}\text { Minor quality } \\
\text { embryo } \\
\text { available } \\
n=86(\%)\end{array}$ & $95 \% \mathrm{Cl}(\%)$ \\
\hline Positive hCG test & $21 \quad(38.2)$ & $25.4-51.0$ & $25(29.1)$ & $20.3-37.9$ \\
\hline Miscarriage & 7 (33.3) & $13.1-53.5$ & $8(32.0)$ & $13.7-50.3$ \\
\hline Pregnancy (12 wks) & $14(25.5)$ & $14.0-37.0$ & $17(19.8)$ & $11.4-28.2$ \\
\hline Live birth & $14(25.5)$ & $14.0-37.0$ & $16(18.6)$ & $10.4-26.8$ \\
\hline
\end{tabular}


Table IV: Clinical outcome of the first cycle of patients in group B receiving ESET or DET

\begin{tabular}{ccccccc}
$\begin{array}{c}\text { eSET, } \\
\mathrm{n}=100(\%)\end{array}$ & $\begin{array}{c}95 \% \mathrm{Cl} \\
(\%)\end{array}$ & & $\begin{array}{c}\mathrm{DET}, \\
\mathrm{n}=74(\%)\end{array}$ & $\begin{array}{c}95 \% \mathrm{Cl} \\
(\%)\end{array}$ \\
\cline { 1 - 3 } \cline { 5 - 6 } & & & & & & \\
45 & $(45.0)$ & $35.2-54.8$ & & $30(40.5)$ & $29.3-51.7$ \\
12 & $(26.7)$ & $0.8-39.6$ & & $3(10.0)$ & $0-20.7$ \\
33 & $(33.0)$ & $23.8-42.2$ & & $27(36.5)$ & $25.5-47.5$ \\
1 & $(3.0)^{a}$ & $0-8.8$ & & $7(25.9)^{a}$ & $9.4-42.4$ \\
33 & $(33.0)$ & $23.8-42.2$ & & $26(35.1)$ & $24.2-46.0$
\end{tabular}

a Numbers with similar superscripts within one row are significantly different $\left(\chi^{2}\right.$ test, $P<0.05$ )

Table V: Clinical outcome of the total treatment for group $A$ and $B$

$\begin{array}{cccc}\text { Group A, } & 95 \% \mathrm{Cl} & \text { Group B, } & 95 \% \mathrm{Cl} \\ \mathrm{n}=141(\%) & (\%) & \mathrm{n}=174(\%) & (\%)\end{array}$

\begin{tabular}{|c|c|c|c|c|c|c|}
\hline \multicolumn{7}{|l|}{ Fresh } \\
\hline OPU & 289 & 2.0/patienta & $1.9-2.2$ & 315 & 1.8/patienta & $1.7-2.0$ \\
\hline eSET & 211 & $(73.0)^{a}$ & $67.9-78.1$ & 136 & $(43.2)^{a}$ & $37.7-48.7$ \\
\hline Positive hCG test & 91 & $(64.5)$ & $56.6-72.4$ & 113 & $(64.9)$ & $57.8-72.0$ \\
\hline Miscarriage & 28 & $(30.8)^{a}$ & $21.3-40.3$ & 20 & $(17.7)^{a}$ & $10.7-24.7$ \\
\hline Pregnancy (12 wks) & 63 & $(44.7)$ & $36.5-52.9$ & 92 & $(52.9)$ & $45.5-60.3$ \\
\hline Twin pregnancy & 5 & $(7.9)$ & $1.2-14.6$ & 12 & $(12.9)$ & $6.1-19.7$ \\
\hline Live birth & 62 & $(44.0)$ & $35.8-52.2$ & 89 & $(51.1)$ & $43.7-58.5$ \\
\hline \multicolumn{6}{|l|}{ cryopreservation } & $34.6-45.4$ \\
\hline \multicolumn{7}{|l|}{ Cryopreserved } \\
\hline Thaw cycles & 112 & $0.8 /$ patient $^{a}$ & $0.7-0.9$ & 85 & $0.5 /$ patient $^{a}$ & $0.4-0.6$ \\
\hline Pregnancy (12 wks) & 26 & $(23.2)$ & 15.4-31.0 & 20 & $(23.5)$ & $14.5-32.5$ \\
\hline Twin pregnancy & 4 & $(15.4)$ & $1.5-29.3$ & 3 & $(15.0)$ & $0-30.6$ \\
\hline \multicolumn{7}{|l|}{ Cumulative } \\
\hline Pregnancy (12 wks) & 89 & $(63.1)$ & $55.1-71.1$ & 112 & $(64.4)$ & $57.3-71.5$ \\
\hline Twin pregnancy & 9 & $(10.1)$ & $3.8-16.4$ & 15 & $(13.4)$ & 7.1-19.7 \\
\hline Live birth & 88 & $(62.4)$ & $54.4-70.4$ & 109 & $(62.6)$ & $55.4-69.8$ \\
\hline
\end{tabular}

a Numbers with similar superscripts within one row are significantly different ( $\chi^{2}$ test, $P<0.05$ ) 
The outcomes of cycles two and three did not differ significantly between the two groups. Despite the significant difference in miscarriage rate after a maximum of three fresh cycles, the cumulative live birth and twin PR per patient were similar for both strategies $(62.4 \%$ and $10.1 \%$ in group $A$ and $62.6 \%$ and $13.4 \%$ in group $B$, Table $V$ ). Only the mean number of fresh and frozen cycles, patients performed before achieving a pregnancy or before having completed the maximum of three cycles without success, was significantly different ( 2.0 and 1.8 fresh cycles per patient and 0.8 and 0.5 frozen cycles per patient in group $A$ and $B$ respectively, Table $V$ ).

Most cryopreserved embryos in patients not achieving a live birth have been thawed during the study period, except for one embryo in group $A$ and two in group B, all from patients who decided to refrain from transfer. In 29 patients achieving a live birth in the study period, embryos are still cryopreserved. Additionally, in two patients from both groups, cryopreserved embryos are still stored as these patients became pregnant spontaneously.

The drop-out rate after the first cycle was significantly different between the two groups. In group A, 3.2\% (2.1\% for medical and $1.1 \%$ for personal reasons) of the non-pregnant patients dropped out compared to $15.4 \%$ in group B (2.9\% for medical and $9.6 \%$ for personal reasons and $2.9 \%$ because of achieving a spontaneous pregnancy). In group B there was no relation between the number of embryos transferred in the first cycle and the dropout rate as $15.5 \%$ dropped out after ESET and $15.2 \%$ after DET. Between the second and third cycle these numbers were $17.5 \%$ (4.8\% for medical and $9.5 \%$ for personal reasons and $3.2 \%$ because of spontaneous pregnancies) for group $A$ and $13.6 \%$ ( $1.7 \%$ for medical and $11.9 \%$ for personal reasons) for group $B$. To analyse whether this difference in drop out rate can influence cumulative outcome data, a life-table analysis was performed. Assuming a live birth rate in patients dropping out of $0 \%$ resulted in a cumulative live birth rate of $64.7 \%$ in group A and $64.1 \%$ in group B. When assuming a similar live birth rate in patients dropping out and those remaining in the study, these data were $66.5 \%$ and $68.6 \%$ respectively (both differences not significant). 


\section{Discussion}

From the results of this study it became apparent that treatment strategies consisting of eSET (group A) or eSET/DET (group B) in the first cycle and eSET/ DET in the second and third cycles in patients $<38$ years led to similar live birth rates. To obtain this result, more cycles per patient were required in group $A$, while the overall twin PR was not significantly reduced in group A. Our results put the policy with eSET in the first cycle, irrespective of the availability of a good quality embryo, at a relative disadvantage, which is remarkable considering that for example in Belgium the legislation has been changed towards an eSET 'irrespective of the availability of a good quality embryo' policy in patients < 36 years (Gordts et al., 2005). Although the overall cumulative live birth rate was similar in our study, the live birth rate in the first cycle was significantly different. This difference might be explained by the fact that approximately half of all human preimplantation embryos may be considered chromosomally abnormal (Coonen et al., 1994; Baart et al., 2006).

These aneuploidies do not affect the lower quality embryos only, but also morphologically normal embryos (Baart et al., 2006; Munne et al., 2006). In the case of eSET, approximately half of the embryos will be chromosomally abnormal and therefore not result in a pregnancy, even when this embryo is of high morphological quality. This can also be seen in Table III, where the transfer of one good or one moderate quality embryo was shown to lead to statistically similar live birth rates. After the transfer of two embryos in DET, there is a higher chance that at least one chromosomally normal embryo will be replaced, increasing the chance for pregnancy and live birth. It may be argued, however, that in eSET there are also two embryos available for transfer (one is transferred as a fresh embryo and the other after freezing and thawing), with a similar chance for at least one chromosomally normal embryo to be transferred as compared to the transfer of two fresh embryos in DET. In the eSET policy, however, part of the cryopreserved embryos will not survive the thawing process and will be lost before transfer. Therefore, although group $A$ and $B$ started with the same number of embryos (6.2 and 6.0 respectively), the net number of embryos available for transfer and therefore the net number of euploid embryos, will be lower in an eSET policy (group A) as compared to an eSET/DET policy (group B). This difference although not significant, did result in one extra cycle per five patients after eSET in our study.

In addition, the overall twin PR could not be reduced with this strategy. The transfer of cryopreserved embryos and the second and third fresh cycle were all performed according to an eSET/DET policy. The resulting twins annihilated the reduction in twin pregnancies after the first fresh cycle. As $44 \%$ of the twins in group A and $20 \%$ of the twins in group B originated 
from the transfer of two frozen/thawed embryos, more attention should be paid to single embryo transfer with these embryos. Furthermore, more studies are needed to define the morphological criteria of cryopreserved embryos suitable for eSET.

Although the miscarriage rate between the two groups, defined as number of pregnancy losses $\leq 12$ weeks of gestation per positive hCG test, did not differ significantly per cycle, there was a trend towards a higher miscarriage rate in group A as compared with group B (respectively $32.6 \%(15 / 46)$ vs $20.0 \%(15 / 75)$ for the first cycle, $24.2 \%(8 / 33)$ vs $11.1 \%$ (3/27) for the second cycle and $45.5 \%(5 / 11)$ and $18.2 \%(2 / 11)$ for the third cycle). Especially in the second and third cycle, the numbers were very small, but they nevertheless resulted in a significantly different cumulative miscarriage rate. The difference in the first cycle miscarriage rate, although not significant, was also reported in RCTs on eSET vs DET (41\% vs $9 \%$ (Gerris et al., 1999) and $30 \%$ vs $20 \%$ per clinical pregnancy (Lukassen et al., 2005). However, other RCTs reported lower miscarriage rates for eSET compared to DET (15\% vs $16 \%$ (Thurin et al. , 2004) and 4\% vs 9\% (Martikainen et al., 2001)). The reason for the difference in miscarriage rate in our study between group $A$ and $B$ remains elusive. As for group $A$ the miscarriage rate is similar for good and moderate quality embryos, embryo quality cannot be regarded as the cause for the difference in miscarriage rate. It can, however, partly be explained by the fact that only positive $\mathrm{hCG}$ tests resulting in a complete loss of the implantation were counted as a miscarriage. The loss of one conceptus in case of DET (vanishing twin) before or after the detection of a fetal heart beat is not included and the chance of losing both embryos in case of DET is smaller as compared to the loss of one embryo in ESET, putting the latter at a disadvantage.

The drop-out rate in the first cycle of group $B$ was significantly higher as compared to A (15.4\% vs $3.0 \%)$. The most prominent cause for drop-out in group $B$ were personal reasons, while almost no couple dropped out in group $A$ for this reason. As in group B the drop-out rate was similar after eSET and DET, the number of embryos transferred cannot explain this difference. A more likely explanation is that the first cycle of group A was in a study setting where patients not obtaining a pregnancy in the first three cycles were offered a fourth cycle free of charge, while in group B it was part of standard care. The drop-out rate in group B (15.4\%) is comparable to the one reported for standard care IVF (26.2\%) (Land et al., 1997). Patients agreeing to participate in the RCT study are possibly more motivated to continue treatment and less likely to drop out. Life-table analysis, using both a minimal and a maximal assumed live birth rate in the drop-out group led to comparable cumulative live birth rates in groups $A$ and $B$. 
From this it can be concluded that the difference in drop-out rate did not influence the comparison of the two transfer strategies.

From this study we conclude that in order to reduce twin PRs, performing ESET irrespective of the availability of a good quality embryo, in all patients $<38$ years, in the first cycle only is not effective. 
CHAPTER 6

\section{Early cleavage is a valuable addition to existing embryo selection parameters: a study using single embryo transfers}

Aafke P.A. Van Montfoort', John C.M. Dumoulin', Arnold D.M. Kester ${ }^{2}$ and Johannes L.H. Evers'

Research Institute Growth \& Development (GROW), 'Department of Obstetrics \& Gynaecology, Academic Hospital Maastricht, ${ }^{2}$ Department of Methodology and Statistics, University of Maastricht, Maastricht, The Netherlands

Published in: Hum Reprod (2004), 19, 2103-2108. 


\section{Abstract}

BACKGROUND: To reduce the twin pregnancy rate (PR), elective single embryo transfer (eSET) is increasingly implemented. Improvement of the results obtained with ESET can be achieved by better selection of the most viable embryo. This study investigated the predictive value of early cleavage (EC) as an additional parameter for selecting the embryo with the highest implantation potential by using data from SETs.

METHODS: Data from 165 SETs were retrospectively evaluated. Cleavage to the 2-cell stage was determined 23-26h after injection or 25-28h after insemination. Selection of the embryo to be transferred was based on cell morphology and cell number on the day of transfer, not on the EC status. Additional information on the predictive value of EC on developmental potential was obtained by analyzing 253 transfers with two embryos (double embryo transfer, DET) and blastocyst formation of 1160 surplus embryos. Logistic regression was used to determine the predictors of pregnancy or blastocyst development.

RESULTS: A significantly higher PR was observed after transfer of single EC embryos compared to single non-EC embryos (46 versus $18 \%$ ). This result was confirmed by the significantly higher PR after DET with two EC embryos as compared to DET with two non-EC embryos (45 versus $25 \%$ ) and the blastocyst formation of EC embryos compared to non-EC embryos (66 versus 40\%). Logistic regression showed that EC is an independent predictor for both pregnancy and blastocyst development in addition to cell morphology and cell number.

CONCLUSIONS: In order to improve the selection of the embryo with the highest implantation potential, selection for transfer should not be based on cell number and morphology on the day of transfer alone, but also on EC status. 


\section{Introduction}

A twin pregnancy is an adverse outcome of an IVF cycle, because of the increased risk for medical complications including prenatal and neonatal mortality and morbidity, preterm delivery, low birth weight and long-term medical and developmental problems (Elster, 2000; ESHRE Capri Workshop, 2000; Olivennes, 2000; Land and Evers, 2003). As a result, the medical costs of a twin pregnancy increase dramatically compared to the costs for a singleton pregnancy (Elster, 2000; Kinzler et al., 2000). In order to prevent this adverse outcome, many recent studies focus on elective single embryo transfer (eSET). The results show that eSET in a selected group of patients leads to an acceptable pregnancy rate (PR) and a reduction in the twin PR (Gerris et al., 1999; Vilska et al., 1999; Martikainen et al., 2001; Gerris et al., 2002).

As eSET is increasingly performed, there is an urgent need for better criteria to select the embryo with the highest implantation potential. In some IVF centres, embryos are cultured until blastocyst stage to make a better selection. However, in several studies, blastocyst transfer has not been demonstrated to be a better alternative for transfer of cleavage stage embryos (Rienzi et al., 2002) and many centres, including our own, still prefer the transfer of the cleavage stage embryo. At present, the common practice of selecting the most viable cleavage stage embryo is based on embryo morphology and developmental stage on the day of transfer (Giorgetti et al., 1995; Ziebe et al., 1997; Van Royen et al., 1999). Embryo morphology is determined by the number, size and shape of blastomeres, the proportion of fragments and in some studies the presence of multinucleated blastomeres (MNB). It has been demonstrated that after 2 days of culture, the 4- or 5-cell stage is the optimal cleavage stage (Ziebe et al., 1997; Van Royen et al., 1999). Embryos at this cleavage stage with little or no fragmentation and an absence of MNBs achieved a higher implantation and PR compared to embryos at another cleavage stage with more fragmentation (Ziebe et al., 1997; Van Royen et al., 1999).

Recently, new parameters to improve embryo selection have been introduced, e.g. zygote morphology and early cleavage (EC). The morphological parameters for zygote quality include the number of nucleolar precursor bodies (NPB) and their distribution in the pronuclei (Scott et al., 2000; Tesarik et al., 2000; Wittemer et al., 2000). EC embryos were defined as those which had cleaved to the 2-cell stage 25-27h after insemination or ICSI (Shoukir et al., 1997; Sakkas et al., 1998; Bos-Mikich et al., 2001; Lundin et al., 2001; Sakkas et al., 2001; Fenwick et al., 2002; Tsai et al., 2002). The conclusion of all these studies was that transfer of EC embryos leads to a higher pregnancy and implantation rate compared to the transfer of non-EC embryos. However, most studies examined transfers of more than one embryo, where only one embryo had to be an EC embryo. As a result, many cycles were included where both early and non- 
EC embryos were transferred. Therefore it remains uncertain whether the pregnancy or implantation can be attributed to the EC embryo (Shoukir et al., 1997; Sakkas et al., 1998; Bos-Mikich et al., 2001; Sakkas et al., 2001; Fenwick et al., 2002; Tsai et al., 2002).

Furthermore, it is still unclear whether EC is an independent predictor for pregnancy or whether it is correlated with other pregnancy predictors e.g. embryo morphology and cell number on the day of transfer. In several studies it has been shown that EC embryos had a significantly higher cell number and a better embryo morphology on the day of transfer, compared to non-EC embryos (Shoukir et al., 1997; Sakkas et al., 1998; Lundin et al., 2001; Sakkas et al. , 2001; Fenwick et al., 2002). The aim of the present study was to assess the predictive value of $\mathrm{EC}$ for pregnancy in relation to embryo quality, i.e. embryo score. Only SET data were analysed. Surplus embryos were used to evaluate embryonic development to the blastocyst stage. Logistic regression was performed to assess the predictive value of EC for both pregnancy and blastocyst development.

\section{Materials and methods}

\section{Patients}

During a 2 year period (May 2001 to June 2003), all IVF and IVF/ICSI cycles performed in the academic hospital Maastricht that fulfilled the following criteria were included in the present study. First, EC of embryos had to be determined between 23 and $26 \mathrm{~h}$ post-injection or 25 and $28 \mathrm{~h}$ postinsemination, the time difference being necessary to compensate for the time difference in early development between IVF- and ICSI-derived embryos (Nagy et al., 1994; Nagy et al., 1998). Second, there had to be at least one normally fertilized (2PN) zygote present. Third, the indication for IVF treatment should not be preimplantation genetic diagnosis (PGD).

During this period, either one or two embryos were transferred. To evaluate the predictive value of EC for pregnancy, data collected from a total of 165 cycles in which only a single embryo was transferred on the second day after oocyte retrieval were analysed. SET was performed when: a) only one embryo was available $(n=30)$; b) a top quality embryo (4-cell, grade 4 and no MNBs) was available, and the woman was aged $<38$ years of age, which leads according to our standard transfer policy to eSET ( $n=$ 77); c) patients agreed to participate in a research project in which one or two embryos were transferred at random irrespective of embryo score ( $n$ $=47)$; d) there were clear medical or socio/psychological reasons for eSET, e.g. uterus anomaly $(n=11)$.

Besides the 165 SET procedures, a total of 253 double embryo transfer (DET) procedures were analyzed, in which either two EC embryos or two non-EC embryos were transferred.

In addition, 1160 surplus embryos originating from 341 cycles remaining 
after transfer and not suitable for freezing because of inferior quality were cultured until day 6 for analyzing the blastocyst formation rate, provided that the patients had signed an informed consent. This study was approved by the local Ethics Commitee.

\section{Ovarian stimulation protocol}

Patients were down-regulated with triptorelin daily S.c. injections (Decapeptyl; Ferring B.V. The Netherlands) or nafarelin intranasally (Synarel; Searle BV, The Netherlands). To stimulate multiple follicular development, recombinant FSH (Puregon, Organon, The Netherlands) was used. Follicle growth was monitored by ultrasound. As soon as at least three follicles were $\geq 18 \mathrm{~mm} 5000$ IU of hCG (Pregnyl, Organon) was given. Ultrasoundguided oocyte retrieval was performed $36 \mathrm{~h}$ after hCG administration.

IVF and ICSI procedure

IVF and ICSI procedures used were as described in detail earlier (Dumoulin et al., 2000; Dumoulin et al., 2001). Oocytes and embryos were cultured individually in $5 \mu$ droplets covered by mineral oil in an atmosphere of $5 \% \mathrm{O}_{2}, 5 \% \mathrm{CO}_{2}$ and $90 \% \mathrm{~N}_{2}$. Culture medium G1.2 (Vitrolife, Sweden) was alternated with Sydney IVF Cleavage media (Cook, Australia) for each consecutive treatment cycle.

Embryo quality assessment and transfer policy

For each embryo originating from a normally fertilized (2PN) oocyte, an embryo score was calculated on day 2 by multiplying the morphological grade (1 to 4 grading system of Bolton et al. (1989) in which grade 4 is morphologically the best) with the number of blastomeres (Steer et al., 1992). As it has been shown that the 4- or 5-cell stage is the optimal cleavage stage at day 2 and that the presence of MNB is deleterious for embryo viability (Ziebe et al., 1997; Van Royen et al., 1999; Van Royen et al. , 2003), the score for these embryos was adjusted as follows: embryos consisting of $>5$ cells were multiplied by 0.9 , embryos showing MNB as a 2-cell embryo on any of the two observation time-points (23-26h post-injection or 25-28h post-insemination and 41-45h post-injection/insemination) were multiplied by 0.5 and embryos showing MNB as a > 2-cell embryo were multiplied by 0.75. The calculation of embryo scores was performed to make a uniform selection of the embryo to be transferred. In our embryo score system, a 4-cell embryo with morphology grade 4 and an absence of MNB receives the highest score of 16.

Embryos with the highest score available on day 2 were transferred irrespective of the EC status.

Cryopreservation of supernumerary embryos was performed on the morning of the third day if one or more embryos had reached the 8-cell stage, and if they were of good morphological quality. Embryos left over after transfer and not suitable for freezing from consenting patients were 
transferred alternately to droplets of G2.2 medium or Sydney IVF Blastocyst medium and cultured until the sixth day after ovum retrieval. Each day developmental stages were recorded.

Pregnancy was determined by a urine hCG test 14 days post transfer. An ongoing pregnancy was defined as the presence of at least one intrauterine gestational sac with fetal cardiac activity at ultrasound examination, 5-6 weeks after ovum retrieval.

\section{Statistical analysis}

Statistical analysis was performed using the SPSS software for Windows version 10.1 (Statistical Package for Social Sciences, Inc., USA). To compare the differences between the EC embryos and non-EC embryos, Student's t-test was used for the continuous variables and the $\chi^{2}$ test was used for binary variables. To evaluate the impact of embryo score and EC on pregnancy and blastocyst development, two models were constructed by logistic regression analysis. Embryo score was a continuous variable ranging from 1 to 16 . EC was a binary variable (EC 1, non-EC $0)$. To take into account the possible interaction between embryo score and EC status, an interaction factor between those two variables was also included as a possible independent variable in the logistic regression. By using the equation derived from the logistic regression analysis, a predicted probability for both pregnancy and blastocyst development can be calculated for every embryo score. Based on this predicted probability, a receiver operating curve (ROC), plotting the true positives against the false positives, was constructed. The area under the receiver operating curve $\left(A \cup C_{R O C}\right)$ was used to summarize the predictive power of the model, ranging from 0 to 1 . A receiver operating curve (ROC) plots the true positives against the false positives. $P<0.05$ was considered significant.

\section{Results}

From the 1134 ovum retrievals performed in the study period, 418 were included in the present study (165 SET and 253 DET). To investigate the predictive value of $\mathrm{EC}$ as an additional parameter for selecting the embryo with the highest implantation potential, the data from the 165 SETs were used. In 71 of these cycles, standard IVF was performed, while in the remaining 94 cycles, ICSI was performed. The proportion of cycles in which ICSI was applied (58\%) was comparable to that of the total IVFprogramme during the study period (54\%). Of all embryos obtained, 39\% of the ICSI embryos and $38 \%$ of the IVF embryos were early cleavers. Of the 165 embryos that were transferred, 97 (59\%) were EC embryos, while $68(41 \%)$ were non-EC embryos. The results including embryo score and PR are shown in Table I. Both embryo score and PR differ significantly between $\mathrm{EC}$ and non-EC embryos. 
To examine the relationship between EC, embryo score and pregnancy, a logistic regression was performed. EC was a significant predictor when adjusted for embryo score (odds ratio $(O R)=3.24 ; P<0.05$ ). The interaction between embryo score and EC status was not significant. In Figure 1 the predicted PRs when transferring an EC embryo or a non-EC embryo are plotted for each embryo score. The ratio between the probability for pregnancy of EC and non-EC embryos decreases with increasing embryo score. The predictive power of this pregnancy model calculated by the $\mathrm{AUC}_{\mathrm{ROC}}$ was 0.68 .

To confirm the positive impact of EC on PR, 253 DETs were analysed (Table II). In 97 cases where two EC embryos were transferred, the PR was significantly higher compared to the 156 transfers where two nonEC embryos were transferred (45 versus $25 \%$ ). In addition, a total of 1160 surplus embryos from 341 cycles, which were examined for EC as well, were cultured until day 6 after ovum pick-up. Table III shows the blastocyst formation rate and embryo score for both groups. Twenty-three percent of the oocytes which were injected cleaved early, compared to $24 \%$ of the inseminated oocytes. The logistic regression analysis for blastocyst development showed that embryo score $(P<0.0001)$ and EC $(P<0.0001)$ were significant predictors. In addition, there was also a significant interaction between these two predictors $(P<0.05)$, which means that the effect of EC on blastocyst development is dependent on the embryo score. The advantage of EC for blastocyst development decreases as the embryo score increases. This can also be seen in Figure 2 where the predicted probabilities for blastocyst development based on the logistic regression model are shown. The ratio between the probability for blastocyst development of EC and non-EC embryos decreases as the embryo score increases. Because of this extra independent variable, the OR of EC also becomes dependent on embryo score (Figure 3). The OR decreases as the embryo score increases. The $\mathrm{AUC}_{\mathrm{ROC}}$ as a measure for the predictive value of this model is 0.72 . 
Table I: Cycle characteristics of single embryo transfers with an early cleavage embryo and single embryo transfers with a non-early cleavage embryo

\begin{tabular}{lccl}
\hline & Early eavage & No early cleavage & $P$ value \\
\hline No. of SET cycles & 97 & 68 & \\
No. of ICSI embryos (\%) & $203(39)$ & $313(61)$ & \\
No. of IVF embryos (\%) & $161(38)$ & $268(62)$ & \\
Female age (mean \pm SD) & $32.9 \pm 2.9$ & $32.9 \pm 3.4$ & 0.7 \\
No. of oocytes (mean \pm SD) & $10.7 \pm 0.7$ & $8.9 \pm 0.8$ & 0.09 \\
Day 2 embryo score (mean & $13.4 \pm 2.1$ & $10.0 \pm 4.5$ & $P<0.0001$ \\
\pm SD) & & & \\
Pregnancy rate (\%) & $45 / 97(46.4)$ & $12 / 68(17.6)$ & $P<0.001$ \\
Ongoing pregnancy rate (\%) & $36 / 97(37.1)$ & $7 / 68(10.3)$ & $P<0.001$ \\
Abortion rate (\%) & $9 / 45(20)$ & $5 / 12(41.7)$ & 0.12 \\
\hline
\end{tabular}

Table II: Cycle characteristics of double embryo transfers with two early cleavage embryos and double embryo transfers with two non-early cleavage embryos

\begin{tabular}{llll}
\hline & Early cleavage & No early cleavage & $P$ value \\
\hline No. of DET cycles & 97 & 156 & \\
Pregnancy rate (\%) & $44 / 97(45)$ & $39 / 156(25)$ & $P<0.001$ \\
\hline
\end{tabular}

Table III: Comparison of characteristics from early cleavage and non-early cleavage surplus embryos

\begin{tabular}{|c|c|c|c|}
\hline & Early cleavage & No early cleavage & $P$ value \\
\hline No. of embryos & 274 & 886 & \\
\hline No. of ICSI embryos (\%) & $145(23)$ & $476(77)$ & \\
\hline No. of IVF embryos (\%) & $129(24)$ & $410(76)$ & \\
\hline $\begin{array}{l}\text { Day } 2 \text { embryo score (mean } \\
\pm \mathrm{SD} \text { ) }\end{array}$ & $7.0 \pm 3.0$ & $5.5 \pm 2.9$ & $P<0.0001$ \\
\hline Blastocyst rate (\%) & $181 / 274(66.1)$ & $352 / 886(39.7)$ & $P<0.0001$ \\
\hline
\end{tabular}




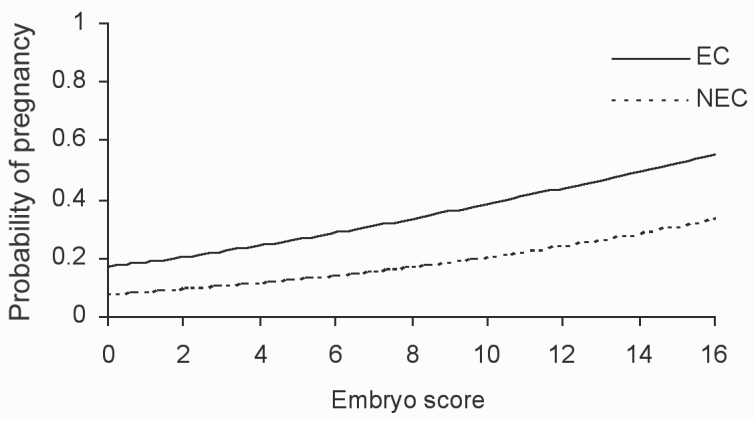

Figure 1: Predicted pregnancy rates for early cleavage (EC) and nonearl cleavage (NEC) embryos derived from a logistic regression model

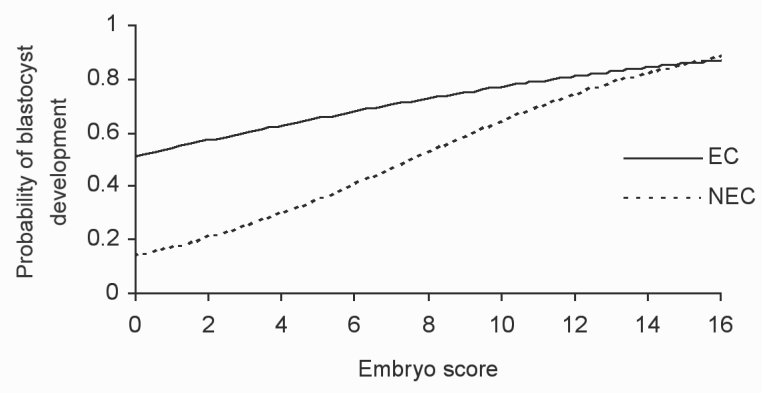

Figure 2: Predicted probability for blastocyst development for early cleavage (EC) and non-early cleavage (NEC) embryos from logisticregression models

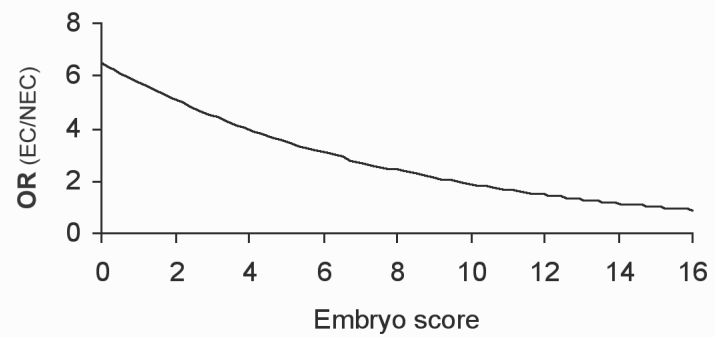

Figure 3: OR for blastocyst development between early cleavage and non-early cleavage embryos in relation to embryo score 


\section{Discussion}

In order to select the most viable embryo, embryo scoring systems have been developed based on embryo morphology and blastomere number on the day of transfer (Cummins et al., 1986; Steer et al., 1992; Giorgetti et al., 1995; Ziebe et al., 1997). Much effort has been devoted to refining existing embryo scoring systems and finding additional simple, noninvasive parameters that could improve the embryo selection procedure (Van Royen et al., 1999; Tesarik et al., 2000; Wittemer et al., 2000). EC status is one of the most promising new parameters.

In previous studies it has been found that transferring embryos that had cleaved early led to a significantly higher PR (Shoukir et al., 1997; Sakkas et al., 1998; Bos-Mikich et al., 2001; Lundin et al., 2001; Sakkas et al., 2001; Fenwick et al., 2002; Tsai et al., 2002) and blastocyst development rate (Fenwick et al., 2002) as compared to non-EC embryos. This is in accordance with the results obtained in the present study (46 versus 18\% for PR after SET, $P<0.001$, and 66 versus $40 \%$ for blastocyst rate, $P<0.0001$ ). However, most studies examined transfers in which two or more embryos were transferred of which at least one embryo had shown EC. This makes it difficult to conclude to which embryo the pregnancy can be attributed. In our study, SET data were analyzed, which makes it possible to determine the relationship between EC and pregnancy arising from one specific embryo. Recently, Salumets et al. (2003) also analyzed SET data; their results for PRs are similar to ours (50 versus $26.4 \%, P=0.001$ ). Furthermore, our results after DET with either two EC embryos or two non-EC embryos (PR 45 versus $25 \%, P<0.001$ ), confirm the findings in the SET group. EC is now used in a few clinics as a selection criterion, but only to make a distinction when more embryos of identical embryo score are available for transfer (Sakkas et al., 1998; Salumets et al., 2003).

Concerning the relation between EC status and embryo quality, it was found previously that, as our results show as well, the early-cleaving embryos had a significantly higher embryo score on the day of transfer (Shoukir et al., 1997; Sakkas et al., 1998; Lundin et al., 2001; Fenwick et al., 2002). Sakkas et al. (1998) already suggested that this might indicate that an EC embryo would have been selected for transfer anyway, based on cell stage and morphology. This would imply that EC status analysis might be redundant. However, as the results from our logistic regression analysis indicate, both embryo score, calculated before embryo transfer, as described in materials and methods, and EC status are independent predictors for both pregnancy and blastocyst development. In the blastocyst model, an interaction exists between embryo score and $\mathrm{EC}$, which is not significant in the pregnancy model. This discrepancy can be explained by the fact that the blastocyst data were more numerous than the pregnancy data. 
Our findings imply that EC status should always be taken into account when selecting the best embryo for transfer and not only when more embryos of identical embryo score are available for transfer. This is not in accordance with the findings of Lundin et al. (2001) who found that, after a logistic regression analysis, EC status was not an independent predictor of pregnancy. However, when the analysis was performed separately for ICSI and IVF, EC was shown to be an independent predictor for birth in ICSI cycles, but not in IVF cycles. They also found that embryos derived after ICSI cleaved earlier compared to those after IVF (Lundin et al., 2001). This has been explained by Nagy et al. (1998). By injecting the spermatozoon into the oocyte, the zona pellucida and cumulus and corona cells barrier is overcome. This gives the ICSI embryos a temporal advantage of $4 \mathrm{~h}$, which might explain why EC was an independent predictor for birth after ICSI, but not after IVF. According to our data this time span was only $2 \mathrm{~h}$ (data not published). That is the reason why the time interval of $2 \mathrm{~h}$ was used between the EC status determination of IVF and ICSI embryos.

The predictive value of the pregnancy model is relatively weak ( $A \cup C=0.68$ ). This is likely due to the fact that there are many other oocyte and embryonic factors which might influence the implantation capacity of the embryo. In addition, a pregnancy is not only dependent on embryo quality, but also on endometrial receptivity, for example.

In our study, it was found that the abortion rate was increased in the non-EC group compared to the EC group (42 versus 20\%). Although the difference is not statistically significant, it is in accordance with the findings of Lundin et al. (2001) who were able to analyse a greater number of cycles.

As in the present study we find that $\mathrm{EC}$ has a predictive value both for implantation after transfer of cleavage stage embryos, as well as for blastocyst formation of surplus embryos, it might be expected that EC also has a predictive value for implantation after transfer of blastocysts. This is, however, not examined in the present study.

In conclusion, this study provided data from a series of SETs showing that EC is a significant predictor of both pregnancy and blastocyst development. Therefore, in order to improve the selection of the embryo with the highest implantation potential, selection of embryos for transfer should not be based on cell number and morphology on the day of transfer alone, but also on EC status. EC stage analysis provides means of improvement of the embryo selection process that might lead to a transfer policy with a higher proportion of SETs. In turn, this will lead to a reduction of the twin PR, which is one of the most serious adverse outcomes of an IVF treatment for both mother and child. 
CHAPTER 7

\section{Differential gene expression in cumulus cells as a prognostic indicator of embryo viability: a microarray analysis}

Aafke P. A. van Montfoort', Joep P.M. Geraedts², John C.M. Dumoulin', Alphons P.M. Stassen ${ }^{3}$, Johannes L.H. Evers' and Torik A.Y. Ayoubi ${ }^{3}$

Research Institute Growth \& Development (GROW), 'Department of Obstetrics \& Gynaecology, '2Department of Clinical Genetics, Academic Hospital Maastricht, The Netherlands and ${ }^{3}$ Department of Population Genetics, Genomics and Bioinformatics, University Maastricht, The Netherlands

Submitted to: Molecular Human Reproduction 


\begin{abstract}
Besides the established selection criteria based on embryo morphology and blastomere number, new parameters for embryo viability are needed to improve the clinical outcome of in vitro fertilization (IVF) and more particular of elective single embryo transfer (eSET). Genome-wide gene expression in cumulus cells was studied, since these cells surround the oocyte inside the follicle and therefore possibly reflect oocyte developmental potential. Early cleavage (EC) was chosen as a parameter for embryo viability. Gene expression in cumulus cells from eight oocytes resulting in an EC embryo (EC-CC; $n=8$ ) and from eight oocytes resulting in a NEC embryo (NEC-CC; $n=8$ ) was analysed using microarrays $(n=16)$. The resulting embryos had all reached the 4-cell stage at day 2 with a similar morphology grade. Between the EC-CC and NEC-CC samples, 611 genes were differentially expressed $(P<0.01)$. These genes are mainly involved in cell cycle, angiogenesis, apoptosis, EGF, FGF and PDGF signaling, general vesicle transport and chemokine and cytokine signaling. To validate the microarray results quantitative real-time PCR (qRT-PCR) of 25 selected genes was performed on the original microarray samples as well as on 24 (12 EC-CC and 12 NEC-CC) extra independent samples Fifteen $(60 \%)$ genes analysed could be validated in the original samples and of these 8 (53\%) could also be validated in independent samples. The most differentially expressed genes among these were CCND2 (cell cycle), CXCR4 (chemokine signaling), GPX3 (peroxidase), CTNND1 (cell adhesion), DHCR7 (steroid metabolism), DVL3 (Wnt signaling), HSPBI (stress response) and TRIM28 (chromatin remodeling). This study shows that the differential expression of several genes in cumulus cells can be related to embryo development. This opens up perspectives for a new molecular embryo or oocyte selection parameter which might also be useful in countries where the selection has to be made at the oocyte stage before fertilization instead of at the embryonic stage.
\end{abstract}




\section{Introduction}

The only way to prevent a dizygotic twin pregnancy in IVF, which is regarded as one of the most serious complications, is single embryo transfer (SET). As most patients have more than one embryo available for transfer, selecting the most viable one is of pivotal importance. Most clinics rely for embryo selection on the non-invasive examination of developmental and morphological aspects of the embryos. In every stage of oocyte and embryonic development, characteristics have been defined which appear to be prognostic indicators of successful pregnancy. Among these are zona pellucida thickness and cytoplasmic granularity of the oocyte, size of pronuclei and alignment of nuclear polar bodies in the zygote, early cleavage in the cleavage stage embryo and number and size of blastomeres, fragmentation and multinucleation in the 4-8 cell stage embryo (see Borini et al. (2005) for a review and Gerris (2005) for a more extensive list of references). Especially early cleavage (EC) appears to be a good parameter for embryo viability as it is highly correlated with the blastocyst formation rate (Fenwick et al., 2002) and the implantation and pregnancy rate (Shoukir et al., 1997; Sakkas et al., 1998; Lundin et al., 2001), not only in double but also in single embryo transfers (Salumets et al., 2003; Van Montfoort et al., 2004). For embryos in the intermediate syngamy state, the pregnancy rate was in between that of EC and nonearly cleavage (NEC) embryos (Wharf et al., 2004).

As the developmental potential of an embryo cannot be fully determined by characteristics visible by microscopy alone, several other markers are studied (Pearson, 2006). For instance, several investigators focussed on the influence of the follicular micro-environment on subsequent embryonic development. The follicular fluid LH and growth hormone levels at the time of oocyte retrieval were higher in embryos with good morphology (Mendoza et al., 2002). Furthermore, the concentrations of hormones (17 $\beta$-estradiol, LH, growth hormone, prolactin, leptin), growth factors (insulin-like growth factor-I), cytokines (interleukin-1) and proteinases (matrix-metalloproteinase-9) in follicular fluid differ according to the probability of pregnancy (Mendoza et al., 2002; Anifandis et al., 2005; Hammadeh et al. , 2005; Lee et al., 2005). Also vascularization of the follicles has been examined as a potential marker for the developmental potential of an embryo. The peri-follicular blood flow characteristics are related to oocyte oxygenation (Van Blerkom et al., 1997) and can differ between the follicles in one ovary. Nargund et al. (1996) found, by Doppler imaging of the follicular blood flow, that oocytes from poorly vascularized follicles developed in morphologically inferior embryos as compared to those from well vascularized follicles. Several other studies confirmed a positive relationship between perifollicular vascularization and pregnancy (Chui et al., 1997; Van Blerkom et al., 1997; Borini et al., 2004). Pregnancies were only achieved with embryos from oocytes which 
had vascularity detected in $>50 \%$ of their follicular circumference and live births only from oocytes with $>75 \%$ follicular vascularity (Chui et al., 1997). Gaulden et al. (1992) suggested that hypoxic intracellular conditions might result in a diminished level of oxidative metabolism in the oocyte and a lower intracellular $\mathrm{pH}$. The latter in turn could lead to meiotic spindle instabilities and chromosomal abnormalities. Indeed, Chiu et al. (1997) and Van Blerkom et al. (1997) reported a significantly higher incidence of aneuploidy and spindle defects in oocytes derived from follicles with poor vascularization as compared to those from well-vascularized follicles. In addition, ATP content of the oocyte and dissolved oxygen content of the follicle fluid are related to oocyte/embryo development (Van Blerkom et al., 1995; Van Blerkom et al., 1997).

As the oocyte is in dialogue with the surrounding cumulus cells via paracrine and gap-junctional signaling (Sutton et al., 2003), we hypothesized that differences in intra-follicular processes which are responsible for oocyte and embryonic development and subsequently implantation are reflected in the gene expression pattern of cumulus cells. The bi-directional communication between the oocyte and the cumulus cells is necessary for oocyte development as oocytes fail to grow in the absence of (a connection with) cumulus cells (Ackert et al., 2001). Zhang et al. (2005) reported that the expression of several genes in cumulus cells, particularly pentraxin 3, was indicative of oocyte and embryo quality. In turn, oocyte factors like growth and differentiation factor-9 (GDF-9) are necessary for cumulus expansion (Sutton et al., 2003).

The aim of this study was to analyse the genome-wide expression of genes in cumulus cells as indicators of embryo viability. By analysing gene expression in cumulus cells, the understanding of the regulation of oogenesis and embryonic development might be improved. This information might lead to new molecular non-invasive embryo selection parameters reflected in cumulus cells that can be used in addition to the existing morphological parameters or might result in an oocyte selection tool for those who are obliged to select a limited number of oocytes for fertilization (Ludwig et al., 2000).

\section{Methods}

Patients and human cumulus cell collection

Patients visiting the IVFclinic of the academic hospital Maastrichtunderwent an IVF or ICSI treatment as described previously (Van Montfoort et al., 2006). For the study, which was approved by the local Ethics Committee, in consenting patients, immediately following ultrasound-guided cumulusoocyte-complex (COC) retrieval, a proportion of the cumulus cells surrounding a single oocyte were removed using a sharp needle, lysed in $100 \mu$ I Trizol reagent (Invitrogen, Carlsbad, USA) supplemented with 1\% (V/ 
v) 2-mercapto-ethanol (Merck, Darmstadt, Germany), snap-frozen in liquid nitrogen and stored at $-80^{\circ} \mathrm{C}$ (cumulus cells from one oocyte per vial). The oocytes were cultured and fertilized individually in $5 \mu$ droplets covered by mineral oil. Between 23-26 h post-injection or 25-28 h post-insemination early cleavage (EC) status of embryos was assessed. A $2 \mathrm{~h}$ time difference is necessary to compensate for the time difference in early development between IVF- and ICSI-derived embryos (Van Montfoort et al., 2004). Subsequently, on day two of development, the embryos were examined for morphology, number of blastomeres and the presence or absence of multinucleated blastomeres (MNBs) (Van Montfoort et al., 2005).

\section{Experimental design}

EC was chosen as a marker for embryo viability. Gene expression in cumulus cells from eight oocytes resulting in an $\mathrm{EC}$ embryo ( $\mathrm{EC}-\mathrm{CC} ; \mathrm{n}=8$ ) and from eight oocytes resulting in a non-EC embryo (NEC-CC; $n=8$ ) derived from six patients were analysed using microarrays $(n=16)$. To exclude a differential gene expression due to differences in patient characteristics, samples were paired. From four patients both an EC-CC and a NEC-CC sample were used. From two additional patients two EC-CC as well as two NEC-CC samples were used. The microarray results were validated by quantitative real-time polymerase chain reaction (qRT-PCR) on the original samples analysed by microarray as well as on 24 'new' samples.

The cumulus cell samples (for microarray and RT-PCR) from EC and NEC embryos, were derived from normally fertilized (2PN) oocytes, which developed into embryos with comparable characteristics on day 2, i.e. 4 -cell with good morphology and no MNBs present.

\section{RNA isolation}

Total RNA was extracted using Trizol reagent (Invitrogen, Carlsbad, USA) according to the manufacturers' instructions with some adaptations for the small quantity of RNA. RNA was precipitated with isopropyl alcohol for $2 \mathrm{~h}$ and the RNA pellet was washed three times with $75 \%$ ethanol. To be able to track the small RNA pellet, $5 \mu \mathrm{g}$ glycogen (Ambion, Woodward, USA) was added to the sample before RNA precipitation. Total RNA was resuspended in $20 \mu \mathrm{l}$ RNAse free water and stored at $-80^{\circ} \mathrm{C}$. For all RNA samples quantity and purity were determined using the Nanodrop ND1000 spectrophotometer (Nanodrop Technologies, Wilmington, USA) and RNA integrity was determined using the Bioanalyzer 2100 (Agilent Technologies, Palo Alto, USA).

Two cycle amplification and microarray hybridization

Fifty $\eta g$ total RNA was amplified using the two-cycle cDNA synthesis kit (Affymetrix, Santa Clara, USA) in combination with the MEGAscript T7 in vitro transcription system (Ambion). Biotin labelled target complementary RNA (CRNA) was fractionated and hybridized to Human Genome U133A 
Plus 2.0 Arrays (Affymetrix). Each array contained more than 54,000 oligonucleotide probe-sets corresponding to 38,500 characterized human genes.

\section{Microarray analysis}

To identify probe sets which were differentially expressed between 8 EC-CC and 8 NEC-CC samples, a three step process was applied. Firstly, Affymetrix GeneChip Operating Software (GCOS, version 1.4) was used to analyze image data. For each transcript represented on the array by a probe set, the expression algorithm computed the detection call (present, absent, or marginal), the detection $p$-value, and the signal which is an average intensity value for each probe set. This resulted in a table with 54,675 probe sets. Secondly, for each probe set the 16 detection calls were used to determine whether the probe set was reliably detected or not and should 104 or should not be selected for further analysis (McClintick and Edenberg, 2006). To this end for every group of eight arrays (the early and the nonearly) the number of present calls was counted (a number ranging from 0 to 8). If six or more calls were present, the probe set was denoted present. If the probe set was in at least one of the two groups denoted present, it was selected for further analysis. Finally, the over- or underexpression of the remaining probe sets in one of the two groups was analyzed using the class comparison method in BRB ArrayTools software package applying a univariate test composed of a paired T-test with random variance model. This was developed by the Biometric Research Branch of the US National Cancer Institute (http://linus.nci.nih.gov/BRB-ArrayTools. html) . Hierarchical clustering of samples was also performed using BRB ArrayTools. Samples were clustered by comparing their expression profiles.

The genes showing significant differential expression between both groups $(P<0.01)$ were classified into functional groups using the Panther classification system (http://www.pantherdb.org) (Thomas et al., 2003). The gene expression data analysis tool (Thomas et al., 2006) was used to determine which biological process or pathway was significantly overexpressed in one of the two groups. This program uses binomial statistics with bonferroni correction to analyse whether the proportion of genes from a certain biological process or pathway present in a gene list (i.e. the list of differentially expressed genes from an array study) is significantly different from the proportion of genes in that process or pathway in the whole human genome $(P<0.05)$.

\section{Quantitative real-time $P C R$}

For the qRT-PCR, TaqMan low density arrays (TLDAs) (Applied Biosystems, Foster City, USA) were used. Each $2 \mu \mathrm{l}$ well of the TLDA contains userdefined primers and probes selected from an online catalogue (http:// myscience.appliedbiosystems.com) for a single gene. One well contains primers and probes for 18S rRNA, a mandatory endogenous control from 
the manufacturer. cDNA was prepared from 100 ng total RNA per sample using the High Capacity cDNA archive kit (Applied Biosystems) according to the manufacturer's instructions. To each cDNA sample (20 $\mu \mathrm{l}), 80$ $\mu \mathrm{l}$ nuclease-free water and $100 \mu \mathrm{l}$ 2x TaqMan Universal PCR Master Mix (Applied Biosystems) was added. This mixture was then equally divided over two sample-loading ports of the TLDA, each connected to one set of all the genes of interest. The arrays were centrifuged twice $\left(1^{\prime}, 331 \mathrm{~g}\right)$ to equally distribute the sample over the wells. Subsequently, the card was sealed to prevent an exchange between wells. QRT-PCR amplification was performed using an Applied Biosystems Prism 7900HT sequence detection system with the following thermal cycler conditions: 2 minutes at $50^{\circ} \mathrm{C}$ and 10 minutes at $94.5^{\circ} \mathrm{C}$, followed by 40 cycles of 30 seconds at $97^{\circ} \mathrm{C}$ and 1 minute at $59.7^{\circ} \mathrm{C}$.

QRT-PCR analysis

The RQ manager 1.2 software was used to generate Ct values corrected for variances in fluorescent signal strength by using a passive reference dye. The geNorm program (Vandesompele et al., 2002) was used to determine the most stably expressed housekeeping genes. Briefly, the average pairwise variation of a housekeeping gene with all other housekeeping genes was calculated. Stepwise exclusion of the gene with the highest variation resulted in a combination of two housekeeping genes that have the most stable expression. The geometrical mean of the Ct values of these two genes was used as a normalization factor which was substracted from the Ct values of the genes of interest to obtain normalized Ct values $(\Delta \mathrm{C} t)$. Subsequently, the mean $\Delta \mathrm{C}$ t of the NEC-CC samples was substracted from the mean $\Delta \mathrm{Ct}$ of the EC-CC samples generating a $\Delta \mathrm{Ct}$. This $\Delta \Delta \mathrm{C} t$ was recalculated into a relative expression quantity $\left(2^{-\Delta \Delta C^{\dagger}}\right)$ of the gene of interest in EC-CC as compared with NEC-CC samples (Livak and Schmittgen, 2001).

\section{Results}

\section{Microarray analysis}

For the gene expression analysis, 8 EC-CC and 8 NEC-CC samples (from 6 patients) have been analysed using 16 microarrays. From the 54,675 probe sets on the array, 18,480 had a present call. Most of these probe sets showed similar expression between the EC-CC and NEC-CC group, except for 737 probe sets that were differentially expressed $(P<0.01)$. For 59 of these probe sets, the corresponding gene is not yet known. Of the 678 remaining probe sets, which correspond to 611 different genes, 162 (24\%) were upregulated and 516 (76\%) were downregulated in EC-CC compared to NEC-CC. Clustering analysis of the arrays based on the 500 
most significantly differentially expressed genes perfectly clustered the ECCC samples and the NEC-CC samples (Figure 1). The two EC-CC samples derived from the same patient (sample 1-EC $a$ and $b$ and sample 6-EC a and $b$ ) and the NEC-CC samples from the same patient (sample 1-NEC a and $b$ and sample 6-NEC $a$ and $b$ ) were highly correlated.

Of the 611 genes that are differentially expressed between EC and NEC, 426 could be categorized into one of the biological functions listed in Table I. Processes significantly overrepresented $(P<0.05$, as compared to the whole human genome) according to the Panther gene expression tool are protein modification, nucleic acid, lipid, fatty acid and steroid metabolism, apoptosis, general vesicle transport, cell cycle, structure and motility, chromatin packaging and remodeling, transport and signaling. The relevant pathways in which these genes are involved are Ras, chemokine and cytokine signaling, EGF, FGF, and PDGF receptor signaling 106 and angiogenesis.

In Table II, to economize space, only the genes ( $n=95)$ with $P<0.001$ are categorized per function. Some genes can be categorized into more than one function or process but in Table II they are listed in their most prominent role. Information on the other differentially expressed genes (with $\mathrm{P}<0.01$ ) can be provided by the authors on request.

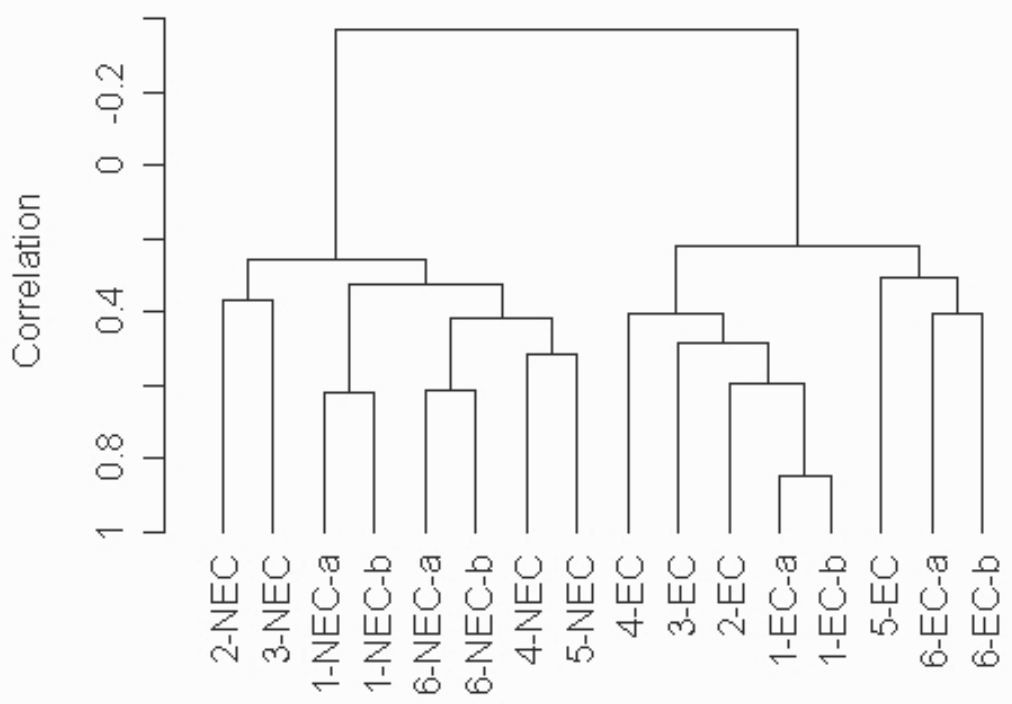

Figure 1: Clustering dendrogram of the 12 samples based on the 500 most significantly differentially expressed genes. 
Table I: Number of significantly differentially expressed genes $(P<0.01)$ up or downregulated in EC-CC as compared to NEC-CC, categorized per functional category (\% per category)

\begin{tabular}{|c|c|c|}
\hline & $\begin{array}{l}\text { Upregu- } \\
\text { lated (\%) }\end{array}$ & $\begin{array}{c}\text { Down } \\
\text { regulated (\%) }\end{array}$ \\
\hline Antioxidation and free radical removal & $0(0)$ & $3(100)$ \\
\hline Apoptosis & $4(31)$ & 9 (69) \\
\hline $\begin{array}{l}\text { Extracellular matrix, cell communication and cell } \\
\text { adhesion }\end{array}$ & $4(16)$ & 21 (84) \\
\hline Cell cycle & $5(38)$ & $8(62)$ \\
\hline DNA metabolism, repair and replication & $3(30)$ & 7 (70) \\
\hline Cell motility and structure & $4(25)$ & $12(75)$ \\
\hline Chromatin packaging and remodeling & $2(20)$ & $8(80)$ \\
\hline Defense & $1(14)$ & $6(86)$ \\
\hline Growth factor & $4(80)$ & 1 (20) \\
\hline Amino acid metabolism and transport & $0(0)$ & 11 (100) \\
\hline Carbohydrate metabolism & $5(26)$ & 14 (74) \\
\hline Lipid, fatty acid and steroid transport and metabolism & $4(22)$ & $14(78)$ \\
\hline Phospholipid metabolism & 1 (14) & $6(86)$ \\
\hline Oxidative phosphorylation & $0(0)$ & $3(100)$ \\
\hline Protein biosynthese & $5(26)$ & $14(74)$ \\
\hline Protein modification & 11 (27) & $30(73)$ \\
\hline Proteolysis & $5(17)$ & $25(83)$ \\
\hline Calcium mediated signaling & 1 (20) & $4(80)$ \\
\hline Cytokine and chemokine mediated signaling & $4(50)$ & $4(50)$ \\
\hline G-protein mediated signaling & $3(23)$ & $10(77)$ \\
\hline Other signaling & $7(21)$ & $26(79)$ \\
\hline Stress response & $0(0)$ & $4(100)$ \\
\hline Transcription factor & $10(20)$ & $40(80)$ \\
\hline mRNA transcription and posttranslational modification & $3(13)$ & $20(87)$ \\
\hline Nucleoside, nucleotide and nucleic acid metabolism & $3(23)$ & $10(77)$ \\
\hline Purine metabolism & $2(22)$ & 7 (78) \\
\hline RNA processing & $0(0)$ & $3(100)$ \\
\hline Cation transport & 4 (33) & 8 (67) \\
\hline Mitochondrial transport & 1 (17) & 5 (83) \\
\hline General vesicle transport & $3(25)$ & $9(75)$ \\
\hline Transporter & $3(15)$ & 17 (85) \\
\hline Other or unknown function & $60(28)$ & $157(72)$ \\
\hline
\end{tabular}


Table II: Genes differentially expressed $(P<0.001)$ in EC-CC vs NEC-CC

Gene ID Gene description

Probe set

Fold ${ }^{a}$

Antioxidation and free radical removal

GPX3

glutathione peroxidase 3 (plasma)

201348_at

0.5

PRDX2

peroxiredoxin 2

39729_at

0.7

Apoptosis

CLU

clusterin (complement lysis inhibitor,

208791_at

0.6

SP-40,40, sulfated glycoprotein 2,

208792_s_at

0.6

testosterone-repressed prostate message

2, apolipoprotein J)

Extracellular matrix, cell communication and cell adhesion

108

CSPG2

chondroitin sulfate proteoglycan 2

211571s at

0.6

CTNND1

(versican)

catenin (cadherin-associated protein),

211240_x_at

0.7

GPC4

delta 1

ITGB 1

glypican 4

204983_s_at

0.6

integrin, beta 1 (fibronectin receptor, beta 1553678_a_at

0.7

polypeptide, antigen CD29 includes MDF2,

MSK12)

\section{Cell cycle}

76P

gamma tubulin ring complex protein (76p

213266_at

0.8

APRIN gene)

CCND2

androgen-induced proliferation inhibitor

cyclin D2

$\mathrm{PRC} 1$

protein regulator of cytokinesis 1

229704_at $\quad 1.3$

200953_s_at $\quad 0.6$

218009_s_at 0.6

DNA metabolism, repair and replication

DKFZP564I0422 THAP domain containing, apoptosis associated protein 2

Cell motility and structure

$\begin{array}{ll}\text { CFL 1 } & \text { cofilin 1 (non-muscle) } \\ \text { MSN } & \text { moesin } \\ \text { PFN1 } & \text { profilin 1 } \\ \text { WASL } & \text { Wiskott-Aldrich syndrom }\end{array}$

WASL Wiskott-Aldrich syndrome-like

$\begin{array}{rr}\text { 1555730_a_at } & 0.6 \\ \text { 200600_at } & 0.7 \\ \text { 200634_at } & 0.6 \\ \text { 205809_s_at } & 1.6\end{array}$

\section{Chromatin packaging and remodeling}

ARIDIA

AT rich interactive domain 1A (SWI- like)

210649_s_at

0.7

Defense

IFITM1

interferon induced transmembrane

214022_s_at

0.7

ILF2

$$
\text { protein } 1 \text { (9-27) }
$$

200052_s_at 0.7 $45 \mathrm{kDa}$ 
Amino acid metabolism and transport

AKAP13

A kinase (PRKA) anchor protein 13

237018_at

0.8

GATM

glycine amidinotransferase (L-arginine:

glycine amidinotransferase)

216733_s_at

Carbohydrate metabolism

C17orf25

chromosome 17 open reading frame 25

209092_s_at

PGD phosphogluconate dehydrogenase

201118_at

0.7

UGP2

UDP-glucose pyrophosphorylase 2

231698 at

1.6

Lipid, fatty acid and steroid transport and metabolism

ACAD8

acyl-Coenzyme A dehydrogenase family,

221669_s_at

DHCR7 member 8

201791_s_at

ELOVL5

ELOVL family member 5, elongation of

PLD3 long chain fatty acids (FEN1/Elo2, SUR4/ Elo3-like, yeast)

1567219_at $\quad 1.5$

201050_at

\section{Protein biosynthese}

$\begin{array}{ll}\text { METAP2 } & \text { methionyl aminopeptidase } 2 \\ \text { RPL14 } & \text { ribosomal protein L14 } \\ \text { RPS3 } & \text { ribosomal protein S3 }\end{array}$

209861_s_at

219138_at

1.5

208692_at

0.8

Protein modification

DNAJB6

DnaJ (Hsp40) homolog, subfamily B,

208810_at

0.7 member 6
OGT
O-linked N-acetylglucosamine (GlcNAc) transferase (UDP-N-acetylglucosamine: polypeptide-N-acetylglucosaminyl transferase)
RYK RYK receptor-like tyrosine kinase
SUMO2 SMT3 suppressor of mif two 3 homolog 2 (yeast)

229787_s_at

\section{Proteolysis}

CST3

cystatin C (amyloid angiopathy and

HTRA 1 cerebral hemorrhage)

201360_at

USP 11 HtrA serine peptidase 1

201185_at

XPNPEP1

ubiquitin specific peptidase 11

208723_at

0.7

$X$-prolyl aminopeptidase (aminopeptidase P) 1, soluble

\section{Cytokine and chemokine mediated signaling}

CXCR4

chemokine (C-X-C motif) receptor 4 


\section{G-protein mediated signaling}

APLP2

amyloid beta (A4) precursor-like protein 2

208703_s_at

0.6

HRB

HIV-1 Rev binding protein

208248_x_at

0.7

(1)

213926_s_at

1.7

\section{Other signaling}

CBL Cas-Br-M (murine) ecotropic retroviral

LMBR1

transforming sequence

limb region 1 homolog (mouse)

MAP3K7

mitogen-activated protein kinase kinase

RAB5B

kinase 7

RAB5B, member RAS oncogene family

$\begin{array}{rr}\text { 229010_at } & 1.6 \\ \text { 224410_s_at } & 0.7 \\ \text { 211536_x_at } & 0.8 \\ \text { 201276_at } & 0.7\end{array}$

\section{Stress response}

HSPB $1 \quad$ heat shock 27kDa protein 1

201841_s_at 0.6

110

\section{Transcription factor}

NFIB

PQBP 1

TRIM28

TSC22D3

WWTR 1 nuclear factor $\mathrm{I} / \mathrm{B}$

polyglutamine binding protein 1

tripartite motif-containing 28

TSC22 domain family, member 3

WW domain containing transcription regulator 1 209289_at

214527_s_at

200990_at

208763_s_at

202132_at
1.4

0.7

0.6

0.8

1.5

\section{mRNA transcription and posttranslational modification}

DHX9

SNRP70

SNRPA
DEAH (Asp-Glu-Ala-His) box polypeptide 9 small nuclear ribonucleoprotein 70kDa polypeptide (RNP antigen) small nuclear ribonucleoprotein polypeptide A

Nucleoside, nucleotide and nucleic acid metabolism

AK1

adenylate kinase 1

HLA-B associated transcript 1

CSNK2A 1

DDX3X

casein kinase 2, alpha 1 polypeptide

DEAD (Asp-Glu-Ala-Asp) box polypeptide 3, X-linked

RANBP9 212107_s_at

1.5

201221_s_at

0.7

201770_at

0.8

\section{Purine metabolism}

DKC 1

dyskeratosis congenita 1, dyskerin

GUK1

LARP 1

guanylate kinase 1

PRPS2

La ribonucleoprotein domain family, member 1 phosphoribosyl pyrophosphate synthetase 2 
Cation transport

solute carrier family 39 (zinc transporter), 217859_s_at

1.3 member 9

\section{General vesicle transport}

ARF6

DCTN 1

RAB6IP2

TRAPPC 1
ADP-ribosylation factor 6

dynactin 1 (p150, glued homolog,

Drosophila)

RAB6 interacting protein 2

trafficking protein particle complex 1

$\begin{array}{rr}\text { 214182_at } & 1.6 \\ \text { 201082_s_at } & 0.7 \\ & \\ \text { 563947_a_at } & 1.5 \\ \text { 225294_s_at } & 0.8\end{array}$

201104 _x_at

0.7

212055_at

0.7

229835_s_at

239999_at

1.4 chromosome 20 open reading frame 45 chromosome 21 open reading frame 34

C21orf34 chromosome 3 open reading frame 10

C3orf10

CKLFSF3

chemokine-like factor superfamily 3

CKSIB

CDC28 protein kinase regulatory subunit 1B

224023_s_at

0.5

1.6

1555705 a at

0.7

201897_s_at

0.8

1554464_a_at

0.7

$212854 \times$ at

0.8

212650 at

1.4

211074 at

208931_s_at

2.2

0.7

ILF3

interleukin enhancer binding factor 3 ,

$90 \mathrm{kDa}$

$\begin{array}{ll}\text { KIAA0256 } & \text { KIAA0256 gene product } \\ \text { KIAA0652 } & \text { KIAA0652 }\end{array}$

LY6E

MEIS4

lymphocyte antigen 6 complex, locus $E$

Meis 1, myeloid ecotropic viral integration

site 1 homolog 4 (mouse)

MGEA5

meningioma expressed antigen 5

(hyaluronidase)

NAG8

nasopharyngeal carcinoma associated

gene protein-8

PMF1

polyamine-modulated factor 1

212451_at

1.4

203364_s_at

0.7

202145 at

0.7

214077_x_at

1.3

223494_at

0.6

210109_at

202337_a†

0.7

regulated in glioma

reticulon 3

221127_s_at

1.5

219549_s_at

0.8

230265_at

1.5

sel-1 suppressor of lin-12-like (C. elegans)

208611_s_at

0.7 fodrin)

SPTAN 1

THO complex 3

224623_at

0.7

205139_s_at

0.7

uronyl-2-sulfotransferase

217821s at

WBP1 1

WW domain binding protein 11

a EC-CC:NEC-CC expression ratio 
QRT-PCR

From 6 out of 8 NEC-CC samples and from all EC-CC samples sufficient RNA remained for a validation of the microarray results by GRT-PCR. Furthermore, an additional 12 EC-CC and 12 NEC-CC samples were analysed to validate the results in independent samples. GRT-PCR was performed on genes selected either because of their highly significant differential expression in both study groups or because of their involvement in a biological relevant pathway or cellular process $(n=25)$. Furthermore, the most stably expressed housekeeping genes from the microarray $(n=4$, EIF4G2, PARK7, SRP14 and RHOA) and 18S (a mandatory control by the manufacturer) were included in the GRT-PCR analysis. SRP14 and RHOA were the two most stably expressed housekeeping genes, i.e. the genes with the lowest variation in expression levels. The expression values of the other 25 genes were normalized against the geometrical mean of these 112 two genes.

In the original samples, analysed by microarray, for 22 out of the 25 selected genes (88\%) the differential expression between EC-CC and NEC-CC could be confirmed by qRT-PCR (Figure 2). When fold changes between 0.9 and 1.1 were excluded 15 of the 25 (60\%) genes could be confirmed. Of these, the differential expression of 10 genes (67\%) could be confirmed in the independent samples of which 8 remained after exclusion of the fold changes between 0.9 and 1.1 (Figure 3). These genes were cyclin D2 (CCND2), catenin delta-1 (CTNND1), CXC chemokine receptor 4 (CXCR4), 7-dehydrocholesterol reductase (DHCR7), dishevelled dsh homolog 3 (DVL3), glutathione peroxidase 3 (GPX3), heatshock $27 \mathrm{kDa}$ protein 1 (HSPB1) and tripartite motif-containing 28 (TRIM28).

\section{Discussion}

To improve the clinical outcome of eSET, the embryo selection needs to be optimized. Besides the established selection criteria based on embryo morphology and blastomere number, new selection parameters should be developed. Information about the oocyte and its development might be a valuable contribution to the existing selection criteria. As cumulus cells surround the oocyte inside the follicle, a microarray analysis was performed on these cells. Both cumulus cells from oocytes developing into an early cleavage embryo (EC-CC) as well as from oocytes developing into a non-early cleavage embryo (NEC-CC) were compared. Our analysis revealed that 18,480 genes were expressed in cumulus cells, 611 of which showed significant differential expression between EC-CC and NECCC. A cluster analysis could perfectly separate the EC-CC and NEC-CC samples, indicating that differences in embryonic implantation potential can already be detected as early as folliculogenesis. 
These differences were not manifested in blastomere number and morphology of the embryo as these were similar in both groups.

The differences in gene expression could not be due to differences in age, ovarian stimulation or other patient characteristics as from each patient one EC-CC and one NEC-CC ( $n=4$ patients) sample or two EC-CC and two NEC-CC samples ( $n=2$ patients) were used. By pairing the samples from each patient, the differential gene expression due to different patient characteristics could be ruled out. 


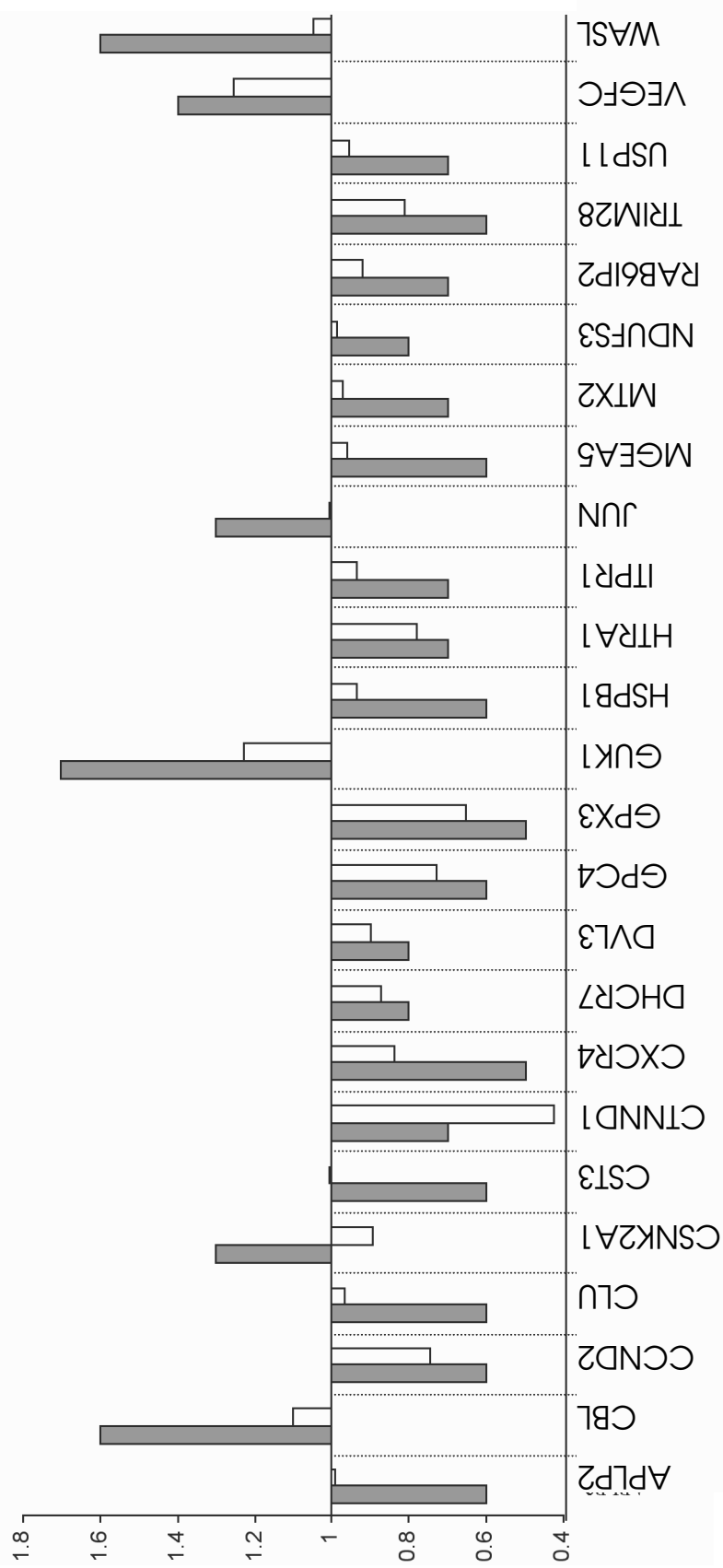

Figure 2: Fold changes (EC-CC vs NEC-CC) of 25 genes measured with microarray analysis (gray bars) and qRT-PCR (white bars) in the same samples. 


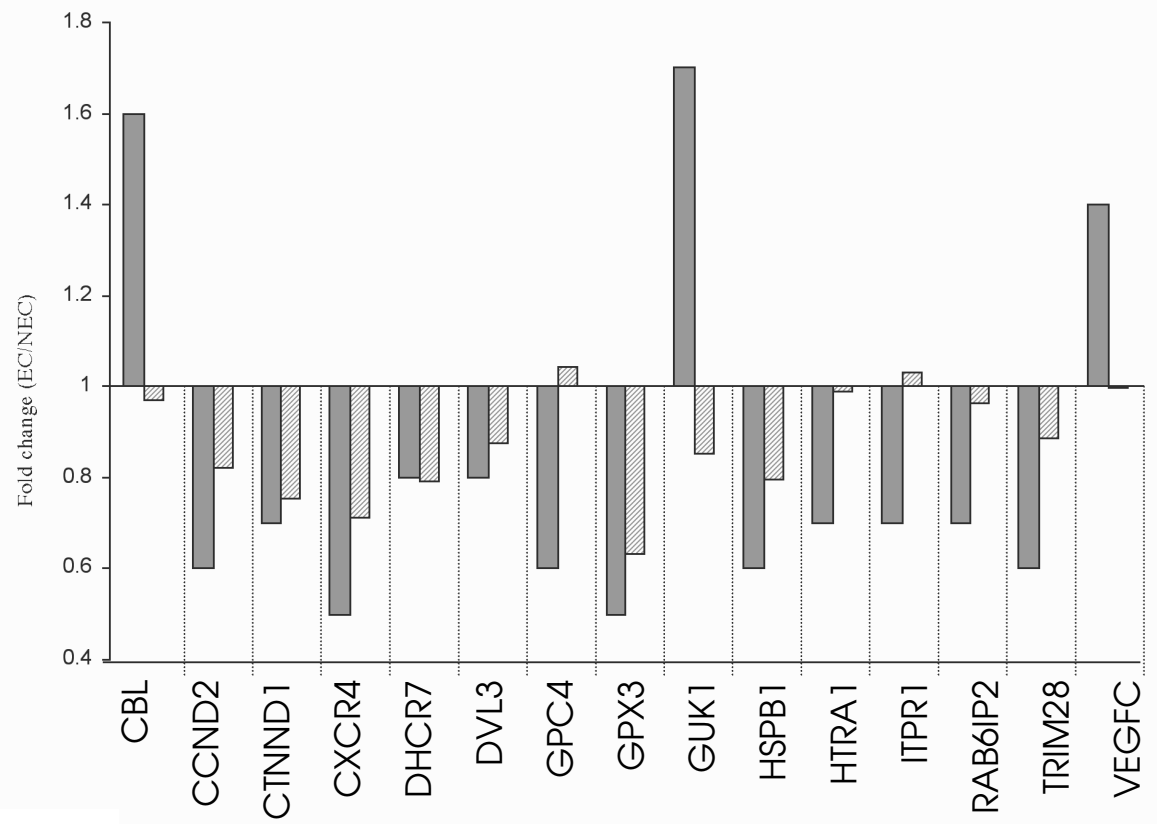

Figure 3: Fold changes (EC-CC vs NEC-CC) of 15 genes measured with microarray analysis (gray bars) and qRT-PCR (striped bars) in different samples.

Furthermore, while other studies analyzing gene expression in human cumulus cells pooled the cumulus cells from several oocytes (Zhang ef al., 2005; Assou et al., 2006), in this study each sample consisted of the cumulus cells from one oocyte. This prevented the loss of information.

Of the 611 differentialy expressed genes $24 \%$ was overexpressed in EC-CC, while $76 \%$ was overexpressed in NEC-CC. The most abundant functions or pathways these genes were involved in were EGF, FGF and PDGF signaling as well as chemokine and cytokine signaling, lipid, fatty acid and steroid metabolism, cell cycle, apoptosis and angiogenesis. Twentyfive genes were selected for validation by quantitative real-time PCR. The gene expression profile found by microarray analysis could be validated for 15 of the 25 (60\%) selected genes. In literature, a $84-88 \%$ concordance between microarray and quantitative real-time PCR has been described (Rajeevan et al., 2001a; Dallas et al., 2005). Microarray results can be influenced by labeling and hybridization efficiency, while quantitative PCR is dependent on the efficiency of the enzymes and primers. 
Especially small fold changes between study groups are sensitive to these variations, which explains the lower concordance in our study (Rajeevan et al., 2001b). The differential expression of 8 of the 15 genes (53\%) could be verified in extra independent samples. This indicates that gene expression validation in independent samples is very important to control for genes not consistently over- or underexpressed in the tested conditions and that with microarray analysis alone some genes can show differential expression between the examined conditions by chance. The validated genes are CCND2, CTNND1, CXCR4, DHCR7, DVL3, GPX3, HSPB1, TRIM28.

CCND2 is an important cell cycle regulator. Cell proliferation of cumulus cells is important as a null mutation in Ccnd2 in mice impairs cumulus cell proliferation and leads to small follicles unable to ovulate (Sicinski et al., 1996). In our study the expression of CCND2 is higher in NEC-CC as 116 compared to EC-CC. As the expression of CCND2 is induced by FSH but is subsequently inhibited by LH (Robker and Richards, 1998; Muniz et al., 2006), an increased expression of CCND2 might indicate a diminished sensitivity to $\mathrm{LH}$ or a reduced transmission of the $\mathrm{LH}$ signaling by the granulosa cells. The increase in CCND2 expression can also be a reaction to compensate for the increase in apoptosis in NEC-CC. In our study, of the 10 differentially expressed genes $(P<0.01)$ classified under apoptosis, 8 point to an increase in apoptosis in the NEC-CC samples (6 pro-apoptotic genes were overexpressed and 2 anti-apoptotic genes were underexpressed as compared to EC-CC). An increase in cumulus cell apoptosis has indeed been associated with immaturity of oocytes and an impaired fertilization (Host et al., 2002) and pregnancy rate (Lee et al., 2001). It is not exactly known how these apoptotic signals exert their negative effect on oocyte and embryo development. The apoptotic signals can easily be transferred from the cumulus cells to the oocyte through the gap junctions. After the LH peak, these junctions are however closed. Host et al. (2002) suggested, therefore, that the timing of gap junction closure and initiation of apoptotic processes in cumulus cells might be important for oocyte and embryo development.

The chemokine receptor CXCR4 was upregulated in NEC-CC samples. The expression of CXCR4 in cumulus cells was confirmed by Hernandez et al. (2006) who localized the protein at the cell surface. CXCR4 in endothelial and cancer cells is expressed via hypoxia inducible factor- (HIF) $1 \alpha$ mediated transcription, which in turn is activated under hypoxic conditions via the phosphatidylinositol 3-kinase (PI 3-kinase) pathway (Schioppa et al., 2003; Staller et al., 2003; Phillips et al., 2005). EGF can also induce PI 3 -kinase signaling and upregulate CXCR4 transcription via HIF- $1 \alpha$ (Phillips et al., 2005). HIF- $1 \alpha$ is a regulator of oxygen homeostasis by functioning as a transcription factor for genes involved in angiogenesis, erythropoiesis, glycolysis and cell proliferation and survival (Semenza, 2002).How CXCR4 
can relieve hypoxic stress in cumulus cells needs further investigation.

Another gene which might indicate a hypoxic environment in NEC-CC samples is GPX3. Hypoxia is a strong transcriptional regulator of this gene through its HIF-1 binding sites. It is the only glutathione peroxidase in which these binding sites have been detected (Bierl et al., 2004). Hypoxia leads to the formation of reactive oxygen species (ROS) which can cause lipid peroxidation, enzyme inactivation and cell damage, resulting in apoptosis (Buttke and Sandstrom, 1994) not only in cumulus cells, but also in the oocyte (Tatemoto et al. , 2000). Both hypoxia (Van Blerkom et al., 1997) and a concentration of ROS above a certain level in follicular fluid have also been negatively associated with embryonic development, pregnancy outcome (Pasqualotto et al., 2004; Das et al., 2006) and a significantly higher incidence of aneuploidy and spindle defects in oocytes (Chui et al., 1997; Van Blerkom et al., 1997). Nowadays some clinics screen the embryos for aneuploidy by fluorescence in situ hybridization (FISH) on one or two blastomeres biopsied from the embryo (Munne et al., 2003). Although our data need further examination, it would be very promising if intrafollicular hypoxia and thus the enhanced chance for aneuploidy in the corresponding embryo can be analysed by using the cumulus cells instead of removing one or two blastomeres from the embryo.

Although the ultimate goal is to find a new parameter predicting a pregnancy, in this study, EC, which has previously been shown to be a good marker for pregnancy, was used as an endpoint. To correlate a differential gene expression directly to pregnancy, only cycles with SET should be included, as with double embryo transfer (DET) it is not known which embryo implanted. This would however make it impossible to perfectly match samples with a positive and samples with a negative pregnancy outcome for several patient characteristics which probably influence gene expression in cumulus cells. Furthermore, as pregnancy or implantation not only depends on embryonic factors, but also on endometrial receptivity for example, a considerable number of extra microarrays would have been needed in order to find significant intrafollicular differences in gene expression. As EC is a good parameter for pregnancy, independent of blastomere number and morphology (Salumets et al., 2003; Van Montfoort et al., 2004), this was chosen as a marker. Besides, there are indications that whether or not an embryo cleaves early is determined during oogenesis as the human embryonic genome is only activated between the four and eight cell stage (Braude et al., 1988) (Eichenlaub-Ritter and Peschke, 2002). This means that the mature oocyte at ovulation must contain the proteins and mRNA necessary for fertilization and the early stages of embryonic development, including the first cleavage division. Several intrafollicular processes might influence the accumulation of these transcripts. Most investigators of EC concluded indeed after eliminating several explanations for EC like differences in oocyte maturity, that EC might be the result of an 
as yet unknown intrinsic oocyte factor (Shoukir et al., 1997; Sakkas et al., 1998; Lundin et al., 2001; Fenwick et al., 2002).

In conclusion, this study provides evidence that embryo viability is reflected in differential gene expression in the cumulus cells. The molecular discrimination of cumulus cells from different oocytes might lead to an improved embryo selection with improved ESET results or might serve as a tool for oocyte selection necessary in countries where not all oocytes are allowed to be fertilized.

\section{Acknowledgements:}

The authors want to thank Bieke Vanherle for her technical assistance 118 and John Baeten and Ronald Bergkamp from Applied Biosystems for their equipment support. Microarray analyses were performed using BRB ArrayTools developed by Dr. Richard Simon and Amy Peng Lam. 
CHAPTER 8

\section{General discussion}


The number of IVF treatments of subfertility throughout Europe is still increasing, as can be learned from the annual ESHRE reports on IVF in Europe (Nyboe Andersen et al., 2004; 2005; 2006). In Finland and Denmark, nowadays 2.9 and $4.2 \%$ of the newborn children are conceived by IVF (Nyboe Andersen et al., 2006). For The Netherlands this figure can only be estimated since no national registry exists for children conceived by IVF. In 2005, 4725 pregnancies were obtained by IVF and ICSI procedures, leading to 3653 ongoing pregnancies (www.nvog.nl). If we estimate these to have led to 3500 live births, of which $81.3 \%$ singletons, $18.5 \%$ twins and $0.2 \%$ triplets (www.nvog.nl), this would result in 4162 children of which $32 \%$ was part of a twin or triplet pregnancy. In 2005, in total 187,910 children were born (statline.cbs.nl), so in The Netherlands $2.2 \%$ of the children were the result of IVF. This IVF procedure is however not without risks and complications, the most important ones being those secondary to multiple pregnancies following multiple embryo transfer. In order to reduce this multiple, and especially twin pregnancy rate (PR), the aim of the thesis was to compare several transfer strategies with ESET from a clinical and a cost-effectiveness point of view and to improve the selection of the single best embryo for eSET.

\section{eSET - clinical outcome}

After a first conservative application of ESET in patients with only one embryo available, medical reasons demanding SET, or patients wishing the transfer of one embryo only (Vilska et al., 1999; Tiitinen et al., 2001), eSET was applied more generally, but always based on female age, embryo quality and cycle rank (Gerris et al., 2002; De Sutter et al., 2003). The limits of these criteria for eSET varied among several studies (see Tables I and II).

In our centre, instead of gradually liberating female age and embryo quality limits for eSET, we investigated whether eSET could be applied regardless of these criteria by performing an RCT on ESET Vs DET in the first cycle of an unselected group of patients (chapter 3). The transfer of one embryo did indeed result in a significant reduction of the twin PR, but at the expense of a $50 \%$ reduction in ongoing PR. Compared to other RCTs in selected patients, the eSET PR in our study was lower, but the difference in PR between eSET and DET was found in all RCTs (Table I). This indicates that the transfer of two embryos will always lead to a higher PR as compared to the transfer of only one embryo.

In several studies the application of an ESET/DET policy, i.e. ESET in a selected good prognosis population and DET in the remaining patients, was analysed (Table II). Despite the liberalization of the limits for eSET, the PR after eSET and DET remained similar. Most clinics apply such an eSET/ DET policy only in cycle one and two (Tiitinen et al., 2003; Gerris et al., 2004; 
Martikainen et al., 2004). From chapter 2 it can be concluded that such a policy is also suitable for the third cycle. Although as expected, the PR in the third cycle was lower as compared to the PR in the first and second cycle, the PR after ESET and DET was similar per cycle. The cumulative overall twin PR in three fresh cycles eSET and DET in the study period July 2000 - December 2001 was still $22.4 \%$, probably due to the fact that the proportion of cycles with eSET was relatively small; $21 \%$ of the cycles with more than one embryo available. After a less stringent selection for eSET in the RCT study-period 2002-2004, in $43 \%$ of the fresh cycles one embryo could be transferred (chapter 5), leading to an ongoing PR of $52.9 \%$ and a twin PR of $12.9 \%$ after a maximum of three fresh cycles eSET/DET.

Although with an eSET/DET policy the PR in the two groups is similar, when two embryos would be transferred in the eSET group, the PR would be higher. To compensate for this difference other strategies were investigated. In chapter 5 besides eSET in the first cycle of all patients, an eSET/DET policy was applied in the second and third cycle. This led to a similar cumulative PR as compared to three cycles eSET/DET, but also to a similar cumulative twin PR, annulling the motive for applying eSET. According to Lukassen ef al. (2005) a second eSET cycle compensates for the decrease in PR in the first cycle. In an RCT on eSET vs DET in their selected patient population (Table I), the live birth rate was $26 \%$ after eSET and $36 \%$ after DET.

When the non-pregnant patients in the ESET group received a second ESET cycle, the cumulative live birth rate increased to $41 \%$. With this strategy, the twin PR remained reduced, but at the expense of an extra cycle for the patients. Thurin et al. published another solution for the reduction in PR, a subsequent cycle with a single frozen-thawed embryo, if available from the initial eSET cycle. After the fresh eSET or DET cycle, the ongoing PRs were $28.5 \%$ vs $44.1 \%$, respectively $(P<0.001)$. When subsequently a single frozen-thawed embryo was transferred in the eSET group, the ongoing PR increased to $37.8 \%$ (compared to $44.1 \%$ after one fresh DET cycle, $P=0.07$ ) (Thurin et al., 2004). The contribution of cryopreserved embryos to the PR after ESET was also confirmed by Tiitinen et al. (2001). However, in both studies it was not taken into account that after DET also embryos were cryopreserved. 


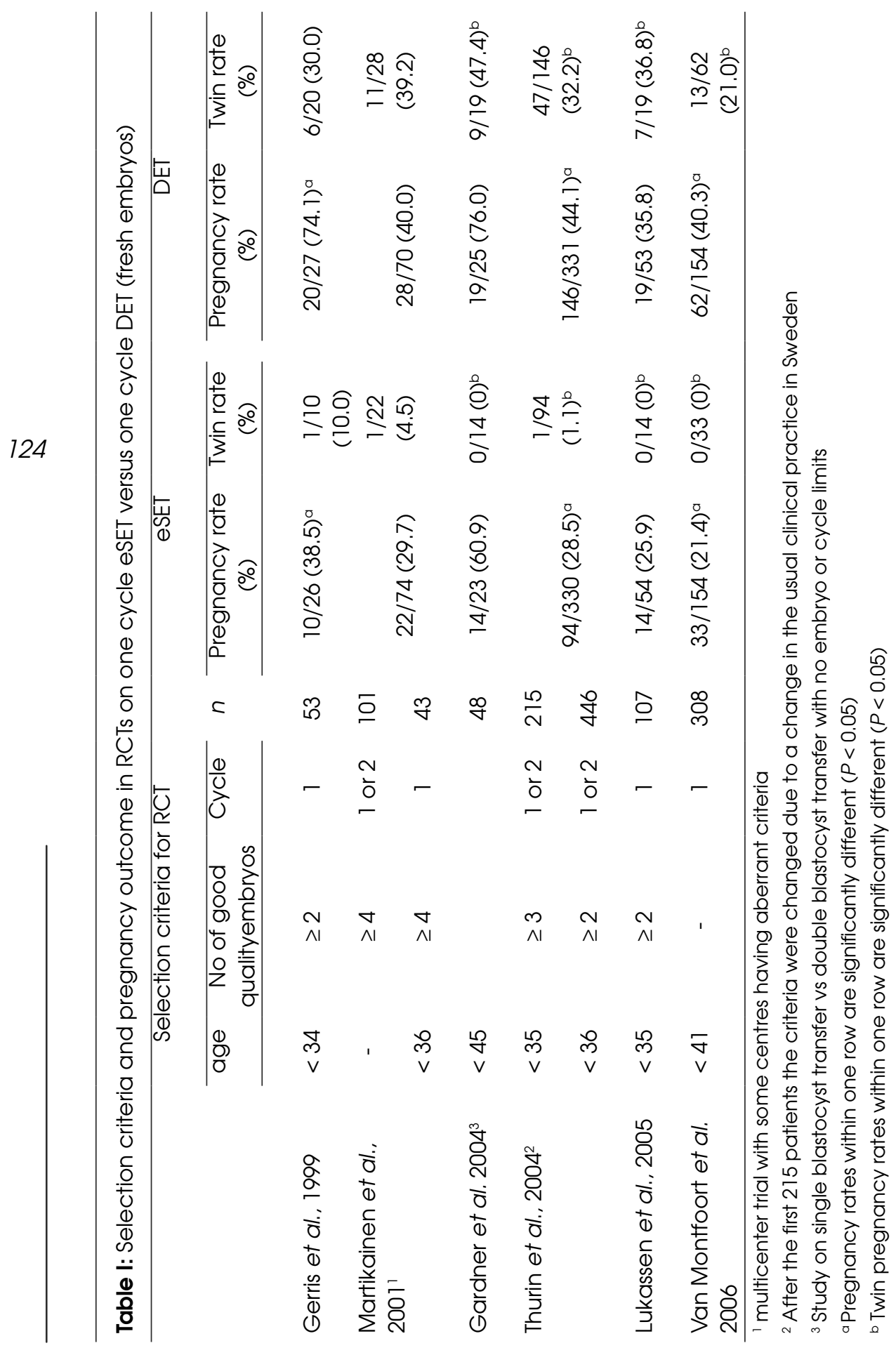




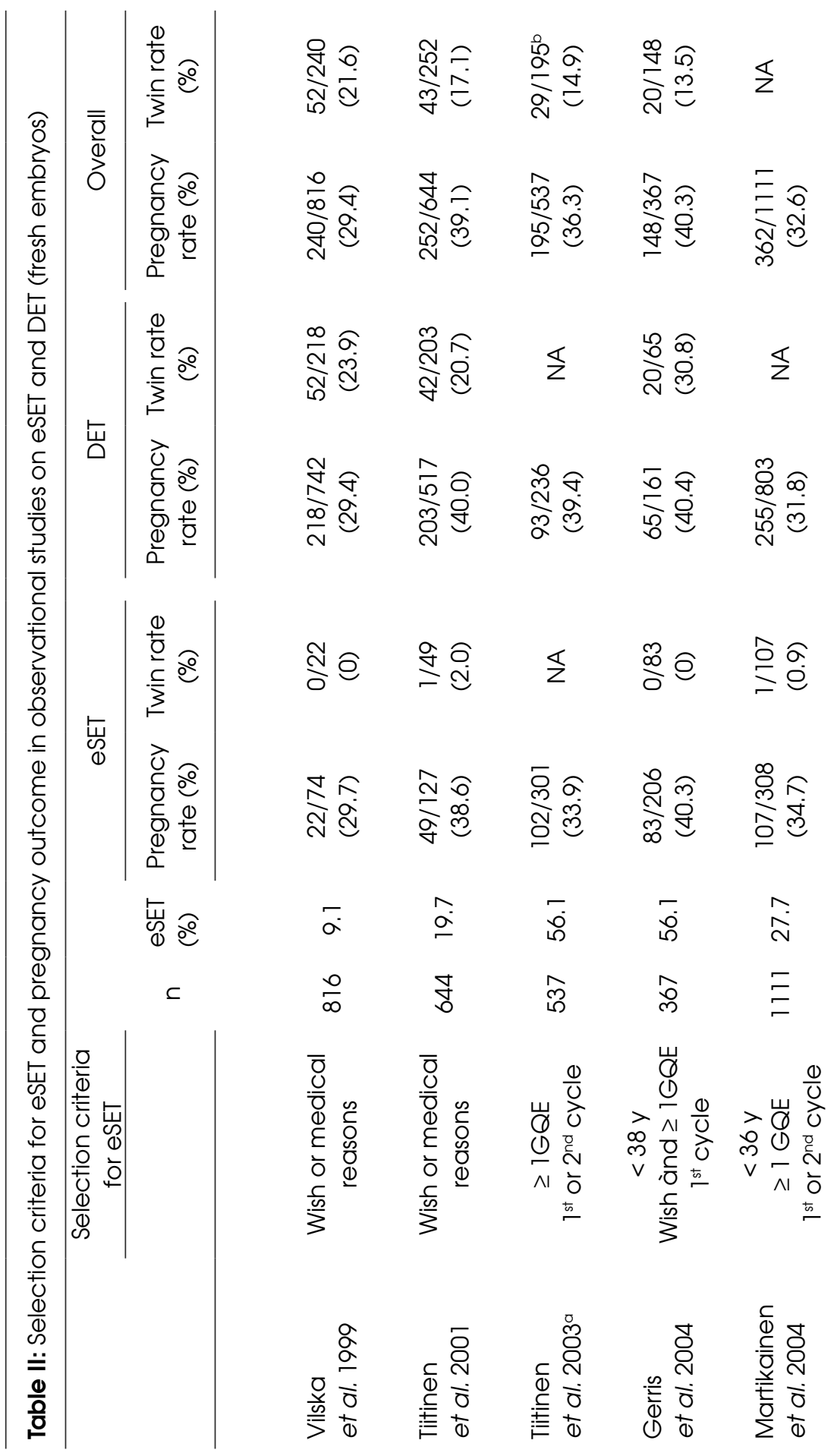




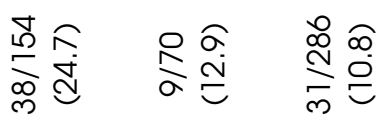

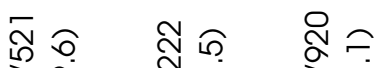

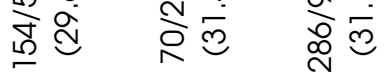

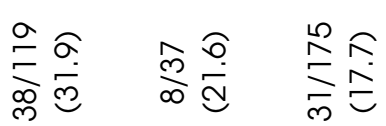

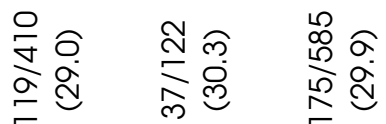

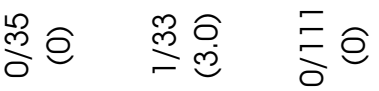

Е

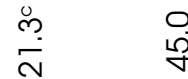

守

్ํำ

ลั ฉ

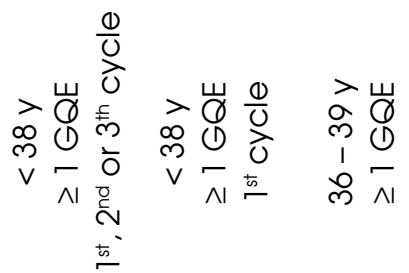

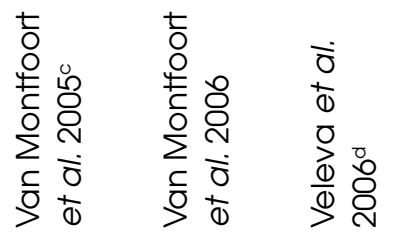

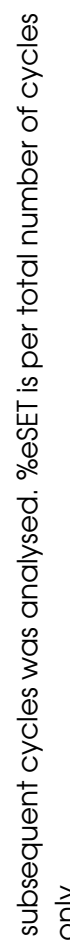

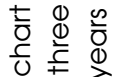

흥 oे

$\varepsilon$ हो

을

O

훙

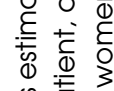

की

可

ते

吸比

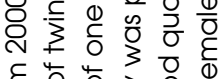

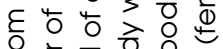

난

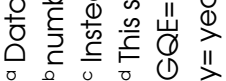




\section{Perspectives on eSET}

As described above, in some clinics all efforts were focussed on maximizing the eSET rate and on how ESET matches optimally in a transfer policy. However, considering the opinions of several actors involved in policy making like the medical practitioners, patients, politicions, health economists and embryologists, it is debatable whether eSET in the majority of patients is the appropriate solution. Opinions from these different actors will be discussed.

\section{Medical perspective}

For the medical practitioners a conflict exists. On one hand, the rate of patients with a live birth after IVF should be as high a possible, which can be achieved by DET. On the other hand IVF clinicians feel also responsible for the well-being of the offspring. The number of multiple pregnancies and the complications thereof should be as low as possible, favouring ESET. As several clinics already apply eSET in a selected group of patients (ranging from 6.9\% in Ireland to 38.7\% in Finland in 2002 (Nyboe Andersen et al., 2006)), medical practitioners indirectly indicate that they accept a lower PR at the benefit of preventing twin pregnancies.

According to the meta-analysis of RCTs on eSET vs DET in a selected group of patients performed by Pandian et al. (2005), medical practitioners accept a live birth rate reduction of $15 \%$ in the patients receiving one embryo by accepting an eSET/DET policy. Assuming that in 50\% of the patients eSET is applied, which is common in some clinics (Tiitinen et al., 2003; Debrock et al., 2005), the live birth rate for the total IVF population will be reduced with $7.5 \%(0.50 \times 0.15)$ and the number of twins will be halved. What is the maximal reduction in PR acceptable for the practitioners?

Based on the conclusions made after observational studies on ESET in a selected group of patients and DET in the remaining part, IVF clinicians are satisfied when the PR is comparable in both groups (Tiitinen et al., 2003; Gerris et al., 2004; Martikainen et al., 2004). However, when the selection limits for eSET are liberalised (e.g. an increased age limit or less strict criteria for a good quality embryo), the proportion of eSET will increase, probably resulting in a decrease in PR. As the pregnancy prognosis of the DET group will decrease as well, this again might result in a comparable PR after ESET and DET, but at a lower level. At which level will this be no longer acceptable? Worldwide, in the majority of clinics, still two or even more embryos are transferred, indicating that in these clinics the number of live births gets priority above the well-being of the offspring. Performing DET in all patients would result in a live birth rate of $39.6 \%$ (chapter 4 ), while in the normal fertile population only $30 \%$ of the conceptions result in a live birth (Macklon et al., 2002). It can be argued whether subfertile patients should be made 'better' than the fertile population. Most of the 'healthy' population achieves a pregnancy from a single embryo. Apparently, as a 
rule, in the human, nature provides for singleton pregnancies. Why should subfertile patients then achieve a pregnancy from multiple embryos? In addition, recent studies show that not only twins born after DET, but also singletons born after DET are at an increased risk of an adverse obstetric outcome (birth weight and gestational age) (Pinborg et al., 2005; De Sutter et al., 2006). This is correlated with the onset of the spontaneous reduction of a twin pregnancy to a singleton pregnancy (i.e. a so-called 'vanishing twin') (Pinborg et al., 2005).

By transfer of only one embryo in the subfertile population, a live birth rate of $20.8 \%$ per cycle can be achieved (chapter 4 ) with a maximal dizygotic twin PR reduction (to $0 \%$ ). As this is not applied in any clinic and in most clinics still two or even more embryos are transferred, practitioners or the possibly more decisive actors described below, apparently have a limit of the reduction in overall PR they accept in order to prevent twin pregnancies and their complications.

\section{Patients' perspective}

A study by Blennborn et al. (2005) on the decision-making between eSET and DET in IVF patients revealed that previous childbirth and the availability of cryopreserved embryos were the most important variables in deciding pro-eSET. Previous failed IVF treatments and an assumed increased PR after DET were variables positively influencing the patients' choice for DET. The importance of PR for patients was confirmed by Murray et al. (2004). According to the answers on a questionnaire in patients starting their first cycle, $30 \%$ of the patients would accept ESET when the PR would be slightly reduced, while $>80 \%$ of the patients would accept eSET when PR remained unchanged. Furthermore, although a twin pregnancy is not regarded as an undesired outcome (67-90\% would not have mind to conceive twins, (Gleicher et al., 1995)), a minority of the patients prefers a twin pregnancy above a singleton pregnancy (20.3\% (Ryan et al., 2004); $41 \%$ (Child et al., 2004)). The desire for a twin is associated with nulliparity, younger patient age, limited knowledge about outcomes of twin pregnancy, lower income and longer duration of infertility (Ryan et al., 2004). Improving the knowledge of patients about the risk and complications of twin pregnancies by an educational campaign enhanced the preference of patients for a singleton pregnancy and for SET (Ryan et al., 2006). However, this theoretical desire for eSET was not translated into action. When these patients were asked to actually have one embryo transferred during their treatment no increase in preference for eSET was seen (Ryan et al., 2006). In contrast, in a study by Gerris et al. (2004), 66\% of the patients chose for an eSET/DET policy instead of DET. Although the PR is similar between eSET with a good quality embryo or DET with moderate quality embryos, when a patient with good quality embryos receives DET the overall PR would be higher. Therefore, it should however be taken into account that studies on patients preferences depend largely on how patients are informed. 
Also reimbursement of an IVF treatment influences the preference for eSET or DET as the preference for eSET increased from 30\% to 50\% when patients were theoretically not charged for any subsequent frozen embryo transfer cycles after fresh eSET (Murray et al., 2004).

The importance of PR and reimbursement for the acceptance of eSET by the patient can also indirectly be concluded from chapter 3. After counseling, a majority of the patients (56\%) agreed to participate in the RCT study on eSET versus DET, where an extra fourth cycle free of charge was offered to compensate for a possible decrease in PR in the eSET arm of the study.

\section{Political perspective}

Health authorities can state their preference for the number of embryos to be transferred through imposing laws on several issues regarding IVF. For instance, in Germany and Italy an oocyte selection has to be made as all produced embryos have to be transferred. The number of embryos may not exceed three and the cryopreservation of supernumerary embryos is prohibited (Benagiano and Gianaroli, 2004). An eSET policy is therefore discouraged. The legislation on insurance coverage of IVF can also (dis)courage eSET. The compensation for the reduction in PR after eSET by an additional cycle will be more easily accepted when these cycles are reimbursed (see patients' perspective). France for example has an unlimited coverage, while almost half of all countries has no coverage (IFFS Surveillance 04, 2004). The most direct manner for health authorities to influence the number of embryos to be transferred is to impose a law on that issue. As the maximum number of embryos to be transferred is subject to wide variation among countries, the political perspective regarding eSET and DET is not uniform (IFFS Surveillance 04, 2004). In some countries the legislation is adjusted to eSET. In Belgium for example, the general aim of the government was to make fertility treatment accessible for more patients with the same financial resources. After a proposal from all IVF centres, besides the medical costs also the laboratory costs are reimbursed on the condition that in the first cycle of all patients $<35$ years eSET is applied (Ombelet, 2004). According to the proposal, the costs of the procedure balance the reduction in costs due to the reduction in twin pregnancies (De Sutter and Gerris, 2006).

In Sweden, the legislation prescribes mandatory eSET, with the exception that two embryos may be transferred if the twinning risk is low (Thurin et al., 2004). This was based on the encouraging reports on eSET, the demands by neonatologists, and for economical reasons (Saldeen and Sundstrom, 2005). The decree is however subject to clinical variation, as the attitude of the clinics towards twinning risk differs.

With only two countries imposing a legislation regarding eSET, politics is not convinced already. Remarkable, the legislation in these countries was changed after a thorough discussion between the authorities and 
the IVF clinicians and neonatologists. This would suggest that politicians in some countries have not changed legislation due to a lack of information and that by informing them about the complications involved in twin pregnancies, they can be convinced to change legislation.

\section{Health economic perspective}

From a health economist's point of view the transfer policy with the lowest costs per live birth is preferred. When regarding an eSET or a DET policy, the difference in cost-effectiveness can be attributed to a difference in live birth rates and costs. The latter is mainly caused by the twin pregnancies in the DET group requiring more examinations and care during the antenatal period, more loss of productivity, an increase in maternal and neonatal hospitalization and considerably higher long term costs (Gerris et al., 2004; Lukassen et al., 2004; Thurin et al., 2004) plus the extra number of cycles in 130 the eSET group necessary for achieving a pregnancy. The most favourable cost-effective strategy will be the one in which the costs of additional cycles in the eSET strategy will balance the costs of a twin pregnancy in the DET strategy.

As described in chapter 4, when eSET is applied in all patients, irrespective of age and embryo quality, the total costs per patient up to 42 weeks after embryo transfer are $€ 7,334$ with a live birth rate of $20.8 \%$. This results in $€ 35,260(7334 / 0.208)$ per live birth. A DET strategy in these patients costs $€ 10,924$ and with a live birth rate of $39.6 \%$ this strategy would cost $€ 27,586(10924 / 0.396)$ per live birth. The ICER of DET compared with SET was $€ 19,096$, meaning that each additional live birth in the DET group, as compared to the eSET group, would cost $€ 19,096$. It depends on what amount of money is regarded as acceptable for an extra live born child to decide whether DET is preferred above ESET from a cost-effectiveness point of view. When ESET or DET was applied in a selected group of patients and if an additional frozen-thawed ESET cycle were to be performed in case no live birth was achieved after ESET, the costs were $€ 9,309$ and $€$ 12,318 for an eSET and DET strategy (Kjellberg et al., 2006). With live birth rates of $38.8 \%$ and $42.9 \%$ this resulted in $€ 23,992$ and $€ 28,713$ per live birth after ESET and DET, respectively. The ICER of DET compared with SET was $€$ 73,307, putting DET at a disadvantage (Kjellberg et al., 2006).

ESET in all patients is not desirable from a health economic point of view. In a selected good prognosis population with an additional frozen-thawed cycle eSET is preferrable to DET. The remaining population has not been taken into account. Therefore it is necessary to calculate whether ESET in the selected good prognosis patients and DET in the remaining patients is still more cost-effective than DET in all patients. Another limitation of all cost-effectiveness studies is that the long-term consequences of eSET and DET are not taken into account. It is obvious that these costs are higher after DET than after eSET, as twins suffer more developmental problems and handicaps than singletons (Luke and Keith, 1992). 
Embryologists' perspective

The challenge of eSET for an embryologist is to select the most appropriate embryo for transfer. Mostly, this selection is based on morphological criteria. At all stages of development criteria were defined to be fulfilled by the qualitatively best embryos (see Borini et al. (2005) for review). The number of blastomeres and morphological aspects are commonly used. The predictive value of some 'new' parameters, like oocyte and polar body I morphology remain controversial (Borini et al., 2005). However early cleavage, the first cleavage of an embryo before 23-26h after injection or before 25-28h after insemination, seems even in single embryo transfers unequivocaly to be a strong predictor, independent of cell number and embro morphology (chapter 6).

In embryo selection it is not only the morphological appearance that characterizes a good quality embryo. Also from within the embryo important information can be obtained, e.g. the metabolic activity of an embryo can be examined by analysing the uptake of nutrients from or the secretion of metabolites into the surrounding medium (Gardner et al., 2001; Van den Bergh et al., 2001). Also the presence of chromosomal aberrations in embryos is a prognostic factor. By removing one or two blastomeres from the embryo, the ploidy state can be determined in these cells by fluorescent in situ hybridization (FISH). Besides that this method is very invasive and detrimental to the embryo, its effectiveness for improving ongoing PR is also being questioned (Twisk et al., 2006)

Therefore a new examination method was put forward: gene expression in cumulus cells (chapter 7). Cumulus cells from oocytes resulting in an early or non-early cleavage embryo with similar morphology grades and cell numbers on the second day of development display a differential gene expression. Although this should be developed further, it indicates that this gene expression may become a valuable marker for embryo quality which is not reflected in the embryo morphology and blastocyst number. Gene expression in cumulus cells might also become a valuable tool for those clinics that have permission to fertilize only a limited number of oocytes. By analysing the cumulus cells, the oocytes with a high embryonic developmental potential can be selected. Hamamah et al. (2006) identified several proteins that are differentially expressed in cumulus cells depending on fertilization of the oocyte. Other studies focus for instance on the concentration of HLA-G in culture medium or the secretion of proteins at a different level between embryos with and without chromosomal defects (Pearson, 2006). Although these are all new methods that need further investigation, they are all non-invasive for the embryo and can lead to a better embryo selection and therefore a better outcome with eSET. 


\section{Summary and conclusion}

Nature has meant the human female to carry singleton pregnancies. The uterus, the cardiovascular system and metabolism are geared towards singletons. To prevent twin pregnancies in IVF, an increasing tendency exists to apply eSET. However, when taking together all factors influencing an ESET policy, it appears that at the moment ESET is not suitable for every patient. Although medical practitioners accept a certain PR reduction for the benefit of creating less twin pregnancies, there is a limit to what is deemed acceptable. This limit is however yet to be defined and apperently differs from country to country (Gleicher et al., 2006).

The patients, on the other hand, want the highest chance of a pregnancy, which would suggest DET. Only when the PR is not reduced, they are prepared to accept an eSET/DET policy. Finally, from a cost-effectiveness point of view, eSET in all patients is not preferable to DET. The costeffectiveness of an ESET/DET policy will be analysed in a companion thesis from our group. For the future, cost-effectiveness analyses should also take the long-term costs of handicaps resulting from twin pregnancies into account since these might ultimately determine the place of assisted reproduction in our health care system.

In conclusion, based on the data obtained so far, an eSET/DET policy is preferable at this moment. The question on what should be the criteria for ESET remains to be solved. In general, a balance has to be found between the decrease in PR with ESET and the decrease in twin PR. A small decrease in PR as compared to DET should be associated with a large decrease in twin PR. It should be carefully considered whether measures decreasing the twin PR only marginally, balance the reduction in PR as only a certain percentage of the twins has complications that should be prevented. Furthermore, a cost-effectiveness analysis should be applied on several transfer strategies, including an ESET/DET policy and including the long-term costs for twins. The patients' knowledge about the risks of a twin pregnancy should be enhanced to increase the acceptance of eSET, at least in a good prognosis subpopulation. And finally, the effectiveness of new non-invasive embryo selection techniques should be examined in order to enhance the PR after ESET. 


\section{References}

Abramov Y, Elchalal U and Schenker JG (1999) Severe OHSS: An 'epidemic' of severe OHSS: a price we have to pay? Hum Reprod 14, 2181-2183.

Abusheikha N, Salha O, Sharma V and Brinsden P (2000) Monozygotic twinning and IVF/ICSI treatment: a report of 11 cases and review of literature. Hum Reprod Update 6, 396-403

Ackert CL, Gittens JE, O'Brien MJ, Eppig JJ and Kidder GM (2001) Intercellular communication via connexin43 gap junctions is required for ovarian folliculogenesis in the mouse. Dev Biol 233, 258-270.

Anifandis G, Koutselini E, Louridas K, Liakopoulos V, Leivaditis K, Mantzavinos T, Sioutopoulou D and Vamvakopoulos N (2005) Estradiol and leptin as conditional prognostic IVF markers. Reproduction 129, 531-534.

Assou S, Anahory T, Pantesco V, Le Carrour T, Pellestor F, Klein B, Reyftmann L, Dechaud H, De Vos J and Hamamah S (2006) The human cumulus--oocyte complex geneexpression profile. Hum Reprod 21, 1705-1719.

Baart EB, Martini E, van den Berg I, Macklon NS, Galjaard RJ, Fauser BC and Van Opstal D (2006) Preimplantation genetic screening reveals a high incidence of aneuploidy and mosaicism in embryos from young women undergoing IVF. Hum Reprod 21, 223-233.

Benagiano $G$ and Gianaroli L (2004) The new Italian IVF legislation. Reprod Biomed Online 9, 117-125.

Bergh C (2005) Single embryo transfer: a mini-review. Hum Reprod 20, 323-327.

Bergh T, Ericson A, Hillensjo T, Nygren KG and Wennerholm UB (1999) Deliveries and children born after in-vitro fertilisation in Sweden 1982-95: a retrospective cohort study. Lancet 354, 1579-1585.

Beurskens MP, Maas JW and Evers JL (1995) Subfertiliteit in Zuid-Limburg: berekening van incidentie en van beroep op specialistische zorg. Ned Tijdschr Geneeskd 139, 235238.

Bierl C, Voetsch B, Jin RC, Handy DE and Loscalzo J (2004) Determinants of human plasma glutathione peroxidase (GPx-3) expression. J Biol Chem 279, 26839-26845.

Blennborn M, Nilsson S, Hillervik C and Hellberg D (2005) The couple's decision-making in IVF: one or two embryos at transfer? Hum Reprod 20, 1292-1297.

Bolton VN, Hawes SM, Taylor CT and Parsons JH (1989) Development of spare human preimplantation embryos in vitro: an analysis of the correlations among gross morphology, cleavage rates, and development to the blastocyst. J In Vitro Fert Embryo Transf 6, 30-35.

Bonduelle M, Liebaers I, Deketelaere V, Derde MP, Camus M, Devroey P and Van Steirteghem A (2002) Neonatal data on a cohort of 2889 infants born after ICSI (1991-1999) and of 2995 infants born after IVF (1983-1999). Hum Reprod 17, 671-694.

Borini A, Tallarini A, Maccolini A, Prato LD and Flamigni C (2004) Perifollicular vascularity monitoring and scoring: a clinical tool for selecting the best oocyte. Eur J Obstet Gynecol Reprod Biol 115 Suppl 1, S102-105.

Borini A, Lagalla C, Cattoli M, Sereni E, Sciajno R, Flamigni C and Coticchio G (2005) Predictive factors for embryo implantation potential. Reprod Biomed Online 10, 653-668. 
Bortolus R, Parazzini F, Chatenoud L, Benzi G, Bianchi MM and Marini A (1999) The epidemiology of multiple births. Hum Reprod Update 5, 179-187.

Bos-Mikich A, Mattos ALG and Ferrari AN (2001) Early cleavage of human embryos: an effective method for predicting succesful IVF/ICSI outcome. Hum Reprod 16, 26582661.

Botting BJ, Davies IM and Macfarlane AJ (1987) Recent trends in the incidence of multiple births and associated mortality. Arch Dis Child 62, 941-950.

Braude P, Bolton V and Moore S (1988) Human gene expression first occurs between the fourand eight-cell stages of preimplantation development. Nature 332, 459-461.

Briggs A and Fenn P (1998) Confidence intervals or surfaces? Uncertainty on the costeffectiveness plane. Health Econ 7, 723-740.

Briggs AH, Wonderling DE and Mooney CZ (1997) Pulling cost-effectiveness analysis up by its bootstraps: a non-parametric approach to confidence interval estimation. Health Econ 6, 327-340.

136 Brouwer WB and Koopmanschap MA (2005) The friction-cost method: replacement for nothing and leisure for free? Pharmacoeconomics 23, 105-111.

Bryan E and Read B (1995) Guidance after twin and singleton death. Arch Dis Child Fetal Neonatal Ed 73, F123.

Buttke TM and Sandstrom PA (1994) Oxidative stress as a mediator of apoptosis. Immunol Today 15, 7-10.

Cahill DJ and Wardle PG (2002) Management of infertility. BMJ 325, 28-32.

Callahan TL, Hall JE, Ettner SL, Christiansen CL, Greene MF and Crowley WF, Jr. (1994) The economic impact of multiple-gestation pregnancies and the contribution of assisted-reproduction techniques to their incidence. N Engl J Med 331, 244-249.

Child TJ, Henderson AM and Tan SL (2004) The desire for multiple pregnancy in male and female infertility patients. Hum Reprod 19, 558-561.

Chui DK, Pugh ND, Walker SM, Gregory L and Shaw RW (1997) Follicular vascularity--the predictive value of transvaginal power Doppler ultrasonography in an in-vitro fertilization programme: a preliminary study. Hum Reprod 12, 191-196.

Coetsier T and Dhont M (1998) Embryo transfer and multiple gestation. Avoiding multiple pregnancies in in-vitro fertilization: who's afraid of single embryo transfer? Hum Reprod 13, 2663-2664.

Cooke ID, Sulaiman RA, Lenton EA and Parsons RJ (1981) Fertility and infertility statistics: their importance and application. Clin Obstet Gynaecol 8, 531-548.

Coonen E, Harper JC, Ramaekers FC, Delhanty JD, Hopman AH, Geraedts JP and Handyside $\mathrm{AH}$ (1994) Presence of chromosomal mosaicism in abnormal preimplantation embryos detected by fluorescence in situ hybridisation. Hum Genet 94, 609-615.

Cummins JM, Breen TM, Harrison KL, Shaw JM, Wilson LM and Hennessey JF (1986) A formula for scoring human embryo growth rates in in vitro fertilization: its value in predicting pregnancy and in comparison with visual estimates of embryo quality. J In Vitro Fert Embryo Transf 3, 284-295.

Dallas PB, Gottardo NG, Firth MJ, Beesley AH, Hoffmann K, Terry PA, Freitas JR, Boag JM, Cummings AJ and Kees UR (2005) Gene expression levels assessed by oligonucleotide microarray analysis and quantitative real-time RT-PCR -- how well do they correlate? BMC Genomics 6, 59. 
Das S, Chattopadhyay R, Ghosh S, Ghosh S, Goswami SK, Chakravarty BN and Chaudhury $\mathrm{K}$ (2006) Reactive oxygen species level in follicular fluid--embryo quality marker in IVF? Hum Reprod 21, 2403-2407.

Daya S (2005) Life table (survival) analysis to generate cumulative pregnancy rates in assisted reproduction: are we overestimating our success rates? Hum Reprod 20, $1135-1143$.

De Neubourg D and Gerris J (2003) Single embryo transfer - state of the art. Reprod Biomed Online 7, 615-622.

De Neubourg D, Gerris J, Mangelschots K, Van Royen E, Vercruyssen M and Elseviers M (2004) Single top quality embryo transfer as a model for prediction of early pregnancy outcome. Hum Reprod 19, 1476-1479.

De Sutter P, Gerris J and Dhont M (2002) A health-economic decision-analytic model comparing double with single embryo transfer in IVF/ICSI. Hum Reprod 17, 28912896.

De Sutter P, Van der Elst J, Coetsier T and Dhont M (2003) Single embryo transfer and multiple pregnancy rate reduction in IVF/ICSI: a 5-year appraisal. Reprod Biomed Online 6, 464-469.

De Sutter P, Delbaere I, Gerris J, Verstraelen H, Goetgeluk S, Van der Elst J, Temmerman M and Dhont M (2006) Birthweight of singletons after assisted reproduction is higher after single- than after double-embryo transfer. Hum Reprod 21, 2633-2637.

De Sutter P and Gerris J (2006) Impact van 'single-embryotransfer'-beleid in België op het aantal meerlinggeboorten en de kosten van fertiliteitsbehandeling. TFO 20, 16-19.

Dean NL, Phillips SJ, Buckett WM, Biljan MM and Tan SL (2000) Impact of reducing the number of embryos transferred from three to two in women under the age of 35 who produced three or more high-quality embryos. Fertil Steril 74, 820-823.

Debrock S, Spiessens C, Meuleman C, Segal L, De Loecker P, Meeuwis L and D'Hooghe TM (2005) New Belgian legislation regarding the limitation of transferable embryos in in vitro fertilization cycles does not significantly influence the pregnancy rate but reduces the multiple pregnancy rate in a threefold way in the Leuven University Fertility Center. Fertil Steril 83, 1572-1574.

Delvigne A and Rozenberg S (2003) Review of clinical course and treatment of ovarian hyperstimulation syndrome (OHSS). Hum Reprod Update 9, 77-96.

Derom R, Vlietinck R, Derom C, Thiery M, Van Maele G and Van den Berghe H (1991) Perinatal mortality in the East Flanders Prospective Twin Survey (preliminary results). Eur J Obstet Gynecol Reprod Biol 41, 25-26.

Dhont M (2001) Single-embryo transfer. Semin Reprod Med 19, 251-258.

Doyle P (1996) The outcome of multiple pregnancy. Hum Reprod 11 Suppl 4, 110-117; discussion 118-120.

Drummond M, Sculpher M, Torrance G, O'Brien B and Stoddart $G$ (2005) Methods for economic health care programmes, 3rd edn. Oxford University Press, Oxford.

Dumoulin JCM, Coonen E, Bras M, Van Wissen LCP, Ignoul-Vanvuchelen RCM, BergersJanssen JM, Derhaag JG, Geraedts JPM and Evers JLH (2000) Comparison of in vitro development of embryos originating from either conventional IVF or ICSI. Hum Reprod 15, 402-409. 
Dumoulin JM, Coonen E, Bras M, Bergers-Janssen JM, Ignoul-Vanvuchelen RC, van Wissen LC, Geraedts JP and Evers JL (2001) Embryo development and chromosomal anomalies after ICSI: effect of the injection procedure. Hum Reprod 16, 306-312.

Dunn A and Macfarlane A (1996) Recent trends in the incidence of multiple births and associated mortality in England and Wales. Arch Dis Child Fetal Neonatal Ed 75, F10-19.

Edgar DH, Bourne H, Jericho H and McBain JC (2000a) The developmental potential of cryopreserved human embryos. Mol Cell Endocrinol 169, 69-72.

Edgar DH, Bourne H, Speirs AL and McBain JC (2000b) A quantitative analysis of the impact of cryopreservation on the implantation potential of human early cleavage stage embryos. Hum Reprod 15, 175-179.

Edwards RG (1996) The history of assisted human conception with especial reference to endocrinology. Exp Clin Endocrinol Diabetes 104, 183-204.

Efron B and Tobshirani R (1993) An introduction to the bootstrap. Chapman \& Hall, New York.

Eichenlaub-Ritter $U$ and Peschke M (2002) Expression in in-vivo and in-vitro growing and maturing oocytes: focus on regulation of expression at the translational level. Hum Reprod Update 8, 21-41.

Eichler HG, Kong SX, Gerth WC, Mavros P and Jonsson B (2004) Use of cost-effectiveness analysis in health-care resource allocation decision-making: how are costeffectiveness thresholds expected to emerge? Value Health 7, 518-528.

Elster N (2000) Less is more: the risks of multiple births. Fertil Steril 74, 617-623.

ESHRE (2002) The European IVF-monitoring programme (EIM), for the European Society of Human Reproduction and Embryology (ESHRE). Assisted reproductive technology in Europe, 1999. Results generated from European registers by ESHRE. Hum Reprod $17,3260-3274$.

ESHRE Campus Course Report (2001) Prevention of twin pregnancies after IVF/ICSI by single embryo transfer. Hum Reprod 16, 790-800.

ESHRE Capri Workshop (2000) Multiple gestation pregnancy. Hum Reprod 15, 1856-1864.

ESHRE CW (2000) Multiple gestation pregnancy. 15, Aug.

Evers JL (2002) Female subfertility. Lancet 360, 151-159.

Fenwick J, Platteau P, Murdoch AP and Herbert M (2002) Time from insemination to first cleavage predicts developmental competence of human preimplantation embryos in vitro. Hum Reprod 17, 407-412.

Fiddelers AAA, Dirksen CD, Van Montfoort APA, Dumoulin JCM, Evers JLH and Severens JL (2005) Economic evaluation of single and double embryo transfer: results of a Markov model. Hum Reprod 20, suppl 1, 142.

Fujii S, Fukui A, Yamaguchi E, Sakamoto T, Sato S and Saito Y (1998) Reducing multiple pregnancies by restricting the number of embryos transferred to two at the first embryo transfer attempt. Hum Reprod 13, 3550-3554.

Gardner DK, Lane M, Stevens J and Schoolcraft WB (2001) Noninvasive assessment of human embryo nutrient consumption as a measure of developmental potential. Fertil Steril 76, 1175-1180.

Gardner DK, Surrey E, Minjarez D, Leitz A, Stevens J and Schoolcraft WB (2004) Single blastocyst transfer: a prospective randomized trial. Fertil Steril 81, 551-555. 
Gardner MO, Goldenberg RL, Cliver SP, Tucker JM, Nelson KG and Copper RL (1995)The origin and outcome of preterm twin pregnancies. Obstet Gynecol 85, 553-557.

Gaulden ME (1992) Maternal age effect: the enigma of Down syndrome and other trisomic conditions. Mutat Res 296, 69-88.

Gerris J, De Neubourg D, Mangelschots K, Van Royen E, Van de Meerssche M and Valkenburg $M$ (1999) Prevention of twin pregnancy after in-vitro fertilization or intracytoplasmatic sperm injection based on strict embryo criteria: a prospective randomized clinical trial. Hum Reprod 14, 2581-2587.

Gerris J, Van Royen E, De Neubourg D, Mangelschots K, Valkenburg M and Ryckaert G (2001) Impact of single embryo transfer on the overall and twin-pregnany rates of an IVF/ ICSI programme. Reproductive BioMedicine Online webpaper 2, 172-177.

Gerris J, De Neubourg D, Mangelschots K, Van Royen E, Vercruyssen M, Barudy-Vasquez J, Valkenburg $M$ and Ryckaert $G$ (2002) Elective single day 3 embryo transfer halves the twinning rate without decrease in the ongoing pregnancy rate of an IVF/ICSI programme. Hum Reprod 17, 2626-2631.

Gerris J, De Sutter P, De Neubourg D, Van Royen E, Vander Elst J, Mangelschots K, Vercruyssen M, Kok P, Elseviers M, Annemans L, et al. (2004) A real-life prospective health economic study of elective single embryo transfer versus two-embryo transfer in first IVF/ICSI cycles. Hum Reprod 19, 917-923.

Gerris JM (2005) Single embryo transfer and IVF/ICSI outcome: a balanced appraisal. Hum Reprod Update 11, 105-121.

Ghai V and Vidyasagar D (1988) Morbidity and mortality factors in twins. An epidemiologic approach. Clin Perinatol 15, 123-140.

Giorgetti C, Terriou P, Auquier P, Hans E, Spach JL, Salzmann J and Roulier R (1995) Embryo score to predict implantation after in-vitro fertilization: based on 957 single embryo transfers. Hum Reprod 10, 2427-2431.

Gleicher N, Campbell DP, Chan CL, Karande V, Rao R, Balin M and Pratt D (1995) The desire for multiple births in couples with infertility problems contradicts present practice patterns. Hum Reprod 10, 1079-1084.

Gleicher N, Weghofer A and Barad D (2006) A formal comparison of the practice of assisted reproductive technologies between Europe and the USA. Hum Reprod 21, 19451950.

Goossens ME, Rutten-van Molken MP, Vlaeyen JW and van der Linden SM (2000) The cost diary: a method to measure direct and indirect costs in cost-effectiveness research. J Clin Epidemiol 53, 688-695.

Gordts S, Campo R, Puttemans P, Brosens I, Valkenburg M, Norre J, Renier M, Coeman D and Gordts S (2005) Belgian legislation and the effect of elective single embryo transfer on IVF outcome. Reprod Biomed Online 10, 436-441.

Gosden R, Trasler J, Lucifero D and Faddy M (2003) Rare congenital disorders, imprinted genes, and assisted reproductive technology. Lancet 361, 1975-1977.

Gronow MJ, Martin MJ, McBain JC, Wein P, Speirs AL and Lopata A (1985) Aspects of multiple embryo transfer. Ann N Y Acad Sci 442, 381-386. 
Hamamah S, Matha V, Berthenet C, Anahory T, Loup V, Dechaud H, Hedon B, Fernandez A and Lamb N (2006) Comparative protein expression profiling in human cumulus cells in relation to oocyte fertilization and ovarian stimulation protocol. Reprod Biomed Online 13, 807-814.

Hammadeh ME, Fischer-Hammadeh C, Amer AS, Rosenbaum P and Schmidt W (2005) Relationship between cytokine concentration in serum and preovulatory follicular fluid and in vitro fertilization/intracytoplasmic sperm injection outcome. Chem Immunol Allergy 88, 80-97.

Hardarson T, Hanson C, Sjogren A and Lundin K (2001) Human embryos with unevenly sized blastomeres have lower pregnancy and implantation rates: indications for aneuploidy and multinucleation. Hum Reprod 16, 313-318.

Helmerhorst FM, Perquin DA, Donker D and Keirse MJ (2004) Perinatal outcome of singletons and twins after assisted conception: a systematic review of controlled studies. Bmj $328,261$.

140 Hernandez-Gonzalez I, Gonzalez-Robayna I, Shimada M, Wayne CM, Ochsner SA, White L and Richards JS (2006) Gene expression profiles of cumulus cell oocyte complexes during ovulation reveal cumulus cells express neuronal and immune-related genes: does this expand their role in the ovulation process? Mol Endocrinol 20, 1300-1321.

Host E, Gabrielsen A, Lindenberg S and Smidt-Jensen S (2002) Apoptosis in human cumulus cells in relation to zona pellucida thickness variation, maturation stage, and cleavage of the corresponding oocyte after intracytoplasmic sperm injection. Fertil Steril 77, 511-515.

Hunault CC, Eijkemans MJC, Pieters MHEC, Te Velde ER, Habbema JDF, Fauser BCJM and Macklon NS (2002) A prediction model for selecting patients undergoing in vitro fertilization for elective embryo transfer. Fertil Steril 77, 725-732.

IFFS Surveillance 04. (2004) Fertil Steril 81 Suppl 4, S9-54.

Jain JK, Boostanfar R, Slater CC, Francis MM and Paulson RJ (2004) Monozygotic twins and triplets in association with blastocyst transfer. J Assist Reprod Genet 21, 103-107.

Khosla S, Dean W, Brown D, Reik W and Feil R (2001) Culture of preimplantation mouse embryos affects fetal development and the expression of imprinted genes. Biol Reprod 64, 918-926.

Kinzler WL, Ananth CV and Vintzileos AM (2000) Medical and economic effects of twin gestations. J Soc Gynecol Investig 7, $321-327$.

Kjellberg AT, Carlsson P and Bergh C (2006) Randomized single versus double embryo transfer: obstetric and paediatric outcome and a cost-effectiveness analysis. Hum Reprod $21,210-216$

Land JA, Courtar DA and Evers JL (1997) Patient dropout in an assisted reproductive technology program: implications for pregnancy rates. Fertil Steril 68, 278-281.

Land JA and Evers JL (2003) Risks and complications in assisted reproduction techniques: Report of an ESHRE consensus meeting. Hum Reprod 18, 455-457.

Landy HJ and Keith LG (1998) The vanishing twin: a review. Hum Reprod Update 4, 177-183.

Lee DM, Lee TK, Song HB and Kim CH (2005) The expression of matrix metalloproteinase-9 in human follicular fluid is associated with in vitro fertilisation pregnancy. Bjog 112 , 946-951. 
Lee KS, Joo BS, Na YJ, Yoon MS, Choi OH and Kim WW (2001) Cumulus cells apoptosis as an indicator to predict the quality of oocytes and the outcome of IVF-ET. J Assist Reprod Genet 18, 490-498.

Levran D, Dor J, Rudak E, Nebel L, Ben-Shlomo I, Ben-Rafael Z and Mashiach S (1990) Pregnancy potential of human oocytes--the effect of cryopreservation. N Engl J Med 323, 1153-1156.

Liebaers I, Bonduelle M, Van Assche E, Devroey P and Van Steirteghem A (1995) Sex chromosome abnormalities after intracytoplasmic sperm injection. Lancet 346 , 1095.

Livak KJ and Schmittgen TD (2001) Analysis of relative gene expression data using real-time quantitative PCR and the 2(-Delta Delta C(T)) Method. Methods 25, 402-408.

Loft A, Petersen K, Erb K, Mikkelsen AL, Grinsted J, Hald F, Hindkjaer J, Nielsen KM, Lundstrom P, Gabrielsen A, et al. (1999) A Danish national cohort of 730 infants born after intracytoplasmic sperm injection (ICSI) 1994-1997. Hum Reprod 14, 2143-2148.

Ludwig M, Schopper B, Katalinic A, Sturm R, Al-Hasani S and Diedrich K (2000) Experience with the elective transfer of two embryos under the conditions of the german embryo protection law: results of a retrospective data analysis of 2573 transfer cycles. Hum Reprod 15, 319-324.

Lukassen HG, Schonbeck Y, Adang EM, Braat DD, Zielhuis GA and Kremer JA (2004) Cost analysis of singleton versus twin pregnancies after in vitro fertilization. Fertil Steril 81 , $1240-1246$.

Lukassen HG, Braat DD, Wetzels AM, Zielhuis GA, Adang EM, Scheenjes E and Kremer JA (2005) Two cycles with single embryo transfer versus one cycle with double embryo transfer: a randomized controlled trial. Hum Reprod 20, 702-708.

Luke B and Keith LG (1992) The contribution of singletons, twins and triplets to low birth weight, infant mortality and handicap in the United States. J Reprod Med 37, 661-666.

Luke B (1994) The changing pattern of multiple births in the United States: maternal and infant characteristics, 1973 and 1990. Obstet Gynecol 84, 101-106.

Lundin K, Bergh C and Hardarson T (2001) Early embryo cleavage is a strong indicator of embryo quality in human IVF. Hum Reprod 16, 2652-2657.

Macklon NS, Geraedts JP and Fauser BC (2002) Conception to ongoing pregnancy: the 'black box' of early pregnancy loss. Hum Reprod Update 8, 333-343.

Magli MC, Jones GM, Gras L, Gianaroli L, Korman I and Trounson AO (2000) Chromosome mosaicism in day 3 aneuploid embryos that develop to morphologically normal blastocysts in vitro. Hum Reprod 15, 1781-1786.

Martikainen H, Tiitinen A, Tomas C, Tapanainen J, Orava M, Tuomivaara L, Vilska S, HydenGranskog C and Hovatta $O$ (2001) One versus two embryo transfer after IVF and ICSI: a randomised study. Hum Reprod 16, 1900-1903.

Martikainen H, Orava M, Lakkakorpi J and Tuomivaara L (2004) Day 2 elective single embryo transfer in clinical practice: better outcome in ICSI cycles. Hum Reprod 19, 13641366.

Martin JA and Park MM (1999) Trends in twin and triplet births: 1980-97. Natl Vital Stat Rep 47, $1-16$. 
McClintick JN and Edenberg HJ (2006) Effects of filtering by Present call on analysis of microarray experiments. BMC Bioinformatics 7, 49.

Mendoza C, Ruiz-Requena E, Ortega E, Cremades N, Martinez F, Bernabeu R, Greco E and Tesarik J (2002) Follicular fluid markers of oocyte developmental potential. Hum Reprod 17, 1017-1022.

Milki AA, Jun SH, Hinckley MD, Behr B, Giudice LC and Westphal LM (2003) Incidence of monozygotic twinning with blastocyst transfer compared to cleavage-stage transfer. Fertil Steril 79, 503-506.

Mittler P (1976) Language development in young twins: biological, genetic and social aspects. Acta Genet Med Gemellol (Roma) 25, 359-365.

Muniz LC, Yehia G, Memin E, Ratnakar PV and Molina CA (2006) Transcriptional regulation of cyclin D2 by the PKA pathway and inducible cAMP early repressor in granulosa cells. Biol Reprod 75, 279-288.

Munne S, Alikani M, Tomkin G, Grifo J and Cohen J (1995) Embryo morphology, developmental rates, and maternal age are correlated with chromosome abnormalities. Fertil Steril 64, 382-391.

Munne S, Sandalinas M, Escudero T, Velilla E, Walmsley R, Sadowy S, Cohen J and Sable D (2003) Improved implantation after preimplantation genetic diagnosis of aneuploidy. Reprod Biomed Online 7, 91-97.

Munne S, Ary J, Zouves C, Escudero T, Barnes F, Cinioglu C, Ary B and Cohen J (2006) Wide range of chromosome abnormalities in the embryos of young egg donors. Reprod Biomed Online 12, 340-346.

Murray S, Shetty A, Rattray A, Taylor V and Bhattacharya S (2004) A randomized comparison of alternative methods of information provision on the acceptability of elective single embryo transfer. Hum Reprod 19, 911-916.

Nagy ZP, Liu J, Joris H, Devroey P and Van Steirteghem A (1994) Time-course of oocyte activation, pronucleus formation and cleavage in human oocytes fertilized by intracytoplasmic sperm injection. Hum Reprod 9, 1743-1748.

Nagy ZP, Janssenswillen C, Janssens R, De Vos A, Staessen C, Van de Velde $H$ and Van Steirteghem AC (1998) Timing of oocyte activation, pronucleus formation and cleavage in humans after intracytoplasmic sperm injection (ICSI) with testicular spermatozoa and after ICSI or in-vitro fertilization on sibling oocytes with ejaculated spermatozoa. Hum Reprod 13, 1606-1612.

Nargund G, Doyle PE, Bourne TH, Parsons JH, Cheng WC, Campbell S and Collins WP (1996) Ultrasound derived indices of follicular blood flow before HCG administration and the prediction of oocyte recovery and preimplantation embryo quality. Hum Reprod 11, 2512-2517.

Norwitz ER, Edusa V and Park JS (2005) Maternal physiology and complications of multiple pregnancy. Semin Perinatol 29, 338-348.

Nyboe Andersen A, Gianaroli L and Nygren KG (2004) Assisted reproductive technology in Europe, 2000. Results generated from European registers by ESHRE. Hum Reprod 19, 490-503.

Nyboe Andersen A, Gianaroli L, Felberbaum R, de Mouzon J and Nygren KG (2005) Assisted reproductive technology in Europe, 2001. Results generated from European registers by ESHRE. Hum Reprod 20, 1158-1176. 
Nyboe Andersen A, Gianaroli L, Felberbaum R, de Mouzon J and Nygren KG (2006) Assisted reproductive technology in Europe, 2002. Results generated from European registers by ESHRE. Hum Reprod 21, 1680-1697.

Nylander PP (1981) The factors that influence twinning rates. Acta Genet Med Gemellol (Roma) 30, 189-202.

Olivennes F (2000) Avoiding multiple pregnancies. Double trouble: yes a twin pregnancy is an advers outcome. Hum Reprod 15, 1663-1665.

Ombelet W (2004) Prevention of multiple pregnancies; the Belgian project. Int Congr Ser $1271,108-111$.

Oostenbrink JB, Koopmanschap MA and Rutten FF (2002) Standardisation of costs: the Dutch Manual for Costing in economic evaluations. Pharmacoeconomics 20, 443-454.

Oostenbrink JB, Bouwmans C, Koopmanschap MA and Rutten FF (2004) Handleiding voor kostenonderzoek, methoden en standaard kostprijzen voor economische evaluaties in de gezondheidszorg. College voor Zorgverzekeringen (Guideline for cost research, methods, and standard cost prices for economic evaluations in health care. Health Care Insurance Board). Diemen.

Pandian Z, Templeton A, Serour $G$ and Bhattacharya S (2005) Number of embryos for transfer after IVF and ICSI: a Cochrane review. Hum Reprod 20, 2681-2687.

Pasqualotto EB, Agarwal A, Sharma RK, Izzo VM, Pinotti JA, Joshi NJ and Rose BI (2004) Effect of oxidative stress in follicular fluid on the outcome of assisted reproductive procedures. Fertil Steril 81, 973-976.

Pearson H (2006) Safer embryo tests could boost IVF pregnancy rates. Nature 444, 12-13.

Pelinck MJ, De Vos M, Dekens M, Van der Elst J, De Sutter P and Dhont M (1998) Embryos cultured in vitro with multinucleated blastomeres have poor implantation potential in human in-vitro fertilization and intracytoplasmic sperm injection. Hum Reprod 13, 960-963.

Petterson B, Nelson KB, Watson L and Stanley F (1993) Twins, triplets, and cerebral palsy in births in Western Australia in the 1980s. BMJ 307, 1239-1243.

Phillips RJ, Mestas J, Gharaee-Kermani M, Burdick MD, Sica A, Belperio JA, Keane MP and Strieter RM (2005) Epidermal growth factor and hypoxia-induced expression of CXC chemokine receptor 4 on non-small cell lung cancer cells is regulated by the phosphatidylinositol 3-kinase/PTEN/AKT/mammalian target of rapamycin signaling pathway and activation of hypoxia inducible factor-1alpha. J Biol Chem 280, 22473-22481.

Pinborg A, Loft A, Schmidt L and Andersen AN (2003) Morbidity in a Danish national cohort of 472 IVF/ICSI twins, 1132 non-IVF/ICSI twins and 634 IVF/ICSI singletons: healthrelated and social implications for the children and their families. Hum Reprod 18, 1234-1243.

Pinborg A, Loft A, Rasmussen S and Nyboe Andersen A (2004) Hospital care utilization of IVF/ ICSI twins followed until 2-7 years of age: a controlled Danish national cohort study. Hum Reprod 19, 2529-2536.

Pinborg A, Lidegaard O, la Cour Freiesleben N and Andersen AN (2005) Consequences of vanishing twins in IVF/ICSI pregnancies. Hum Reprod 20, 2821-2829. 
Rajeevan MS, Ranamukhaarachchi DG, Vernon SD and Unger ER (2001a) Use of real-time quantitative PCR to validate the results of CDNA array and differential display PCR technologies. Methods 25, 443-451.

Rajeevan MS, Vernon SD, Taysavang N and Unger ER (2001b) Validation of array-based gene expression profiles by real-time (kinetic) RT-PCR. J Mol Diagn 3, 26-31.

Rienzi L, Ubaldi F, lacobelli M, Ferrero S, Minasi MG, Martinez F, Tesarik J and Greco E (2002) Day 3 embryo transfer with combined evaluation at the pronuclear and cleavage stages compares favourably with day 5 blastocyst transfer. Hum Reprod 17, 18521855.

Robker RL and Richards JS (1998) Hormone-induced proliferation and differentiation of granulosa cells: a coordinated balance of the cell cycle regulators cyclin D2 and p27Kipl. Mol Endocrinol 12, 924-940.

Ryan GL, Zhang SH, Dokras A, Syrop CH and Van Voorhis BJ (2004) The desire of infertile patients for multiple births. Fertil Steril 81, 500-504.

144 Ryan GL, Sparks AET, Sipe CS, Dokras A, Syrop CH and Van Voorhis BJ (2006) Educational campaign improves knowledge of twin risks in IVF patients but may not impact number of embryos transferred. Hum Reprod 21, suppl 1, i98.

Sakkas D, Shoukir Y, Chardonnens D, Grace Bianchi P and Campana A (1998) Early cleavage of human embryos to the two-cell stage after intracytoplasmic sperm injection as an indicator of embryo viability. Hum Reprod 13, 182-187.

Sakkas D, Percival G, D'Arcy Y, Sharif K and Afnan M (2001) Assessment of early cleaving in vitro fertilized human embryos at the 2-cell stage before transfer improves embryo selection. Fertil Steril 76, $1150-1156$.

Saldeen P and Sundstrom P (2005) Would legislation imposing single embryo transfer be a feasible way to reduce the rate of multiple pregnancies after IVF treatment? Hum Reprod 20, 4-8.

Salumets A, Hyden-Granskog C, Suikkari A, Tiitinen A and Tuuri T (2001) The predictive value of pronuclear morphology of zygotes in the assessment of human embryo quality. Hum Reprod 16, 2177-2181.

Salumets A, Hyden-Granskog C, Makinen S, Suikkari A, Tiitinen A and Tuuri T (2003) Early cleavage predicts the viability of human embryos in elective single embryos transfer procedures. Hum Reprod 18, 821-825.

Santema JG, Koppelaar I and Wallenburg HC (1995) Hypertensive disorders in twin pregnancy. Eur J Obstet Gynecol Reprod Biol 58, 9-13.

Schachter M, Raziel A, Friedler S, Strassburger D, Bern O and Ron-El R (2001) Monozygotic twinning after assisted reproductive techniques: a phenomenon independent of micromanipulation. Hum Reprod 16, 1264-1269.

Schioppa T, Uranchimeg B, Saccani A, Biswas SK, Doni A, Rapisarda A, Bernasconi S, Saccani S, Nebuloni M, Vago L, et al. (2003) Regulation of the chemokine receptor CXCR4 by hypoxia. J Exp Med 198, 1391-1402.

Scott L, Alvero R, Leondires M and Miller B (2000) The morphology of human pronuclear embryos is positively related to blastocyst development and implantation. Hum Reprod 15, 2394-2403.

Scott LA and Smith S (1998) The successful use of pronuclear embryo transfers the day following oocyte retrieval. Hum Reprod 13, 1003-1013. 
Sebire NJ, Jolly M, Harris J, Nicolaides KH and Regan L (2001) Risks of obstetric complications in multiple pregnancies: an analysis of more than 400000 pregnancies in the UK. Prenat Neonat Med 6, 89-94.

Semenza $G$ (2002) Signal transduction to hypoxia-inducible factor 1. Biochem Pharmacol 64, 993-998.

Senat MV, Ancel PY, Bouvier-Colle MH and Breart $G$ (1998) How does multiple pregnancy affect maternal mortality and morbidity? Clin Obstet Gynecol 41, 78-83.

Severens JL, Brunenberg DE, Fenwick EA, O'Brien B and Joore MA (2005) Cost-effectiveness acceptability curves and a reluctance to lose. Pharmacoeconomics 23, 12071214.

Shoukir Y, Campana A, Farley T and Sakkas D (1997) Early cleavage of in-vitro fertilized human embryos to the 2-cell stage: a novel indicator of embryo quality and viability. Hum Reprod 12, 1531-1536.

Sibai BM, Hauth J, Caritis S, Lindheimer MD, MacPherson C, Klebanoff M, VanDorsten JP, Landon M, Miodovnik M, Paul R, et al. (2000) Hypertensive disorders in twin versus singleton gestations. National Institute of Child Health and Human Development Network of Maternal-Fetal Medicine Units. Am J Obstet Gynecol 182, 938-942.

Sicinski P, Donaher JL, Geng Y, Parker SB, Gardner H, Park MY, Robker RL, Richards JS, Mc Ginnis LK, Biggers JD, et al. (1996) Cyclin D2 is an FSH-responsive gene involved in gonadal cell proliferation and oncogenesis. Nature 384, 470-474.

Slotnick RN and Ortega JE (1996) Monoamniotic twinning and zona manipulation: a survey of U.S. IVF centers correlating zona manipulation procedures and high-risk twinning frequency. J Assist Reprod Genet 13, 381-385.

Speirs AL, Lopata A, Gronow MJ, Kellow GN and Johnston WI (1983) Analysis of the benefits and risks of multiple embryo transfer. Fertil Steril 39, 468-471.

Spellacy WN, Handler A and Ferre CD (1990) A case-control study of 1253 twin pregnancies from a 1982-1987 perinatal data base. Obstet Gynecol 75, 168-171.

Staessen C, Janssenswillen C, Van den Abbeel E, Devroey P and Van Steirteghem AC (1993) Avoidance of triplet pregnancies by elective transfer of two good quality embryos. Hum Reprod 8, 1650-1653.

Staessen C, Platteau P, Van Assche E, Michiels A, Tournaye H, Camus M, Devroey P, Liebaers I and Van Steirteghem A (2004) Comparison of blastocyst transfer with or without preimplantation genetic diagnosis for aneuploidy screening in couples with advanced maternal age: a prospective randomized controlled trial. Hum Reprod 19, 2849-2858.

Staller P, Sulitkova J, Lisztwan J, Moch H, Oakeley EJ and Krek W (2003) Chemokine receptor CXCR4 downregulated by von Hippel-Lindau tumour suppressor PVHL. Nature 425, 307-311.

Steer CV, Mills CL, Tan SL, Campbell S and Edwards RG (1992) The cumulative embryo score: a predictive embryo scoring technique to select the optimal number of embryos to transfer in an in-vitro fertilization and embryo transfer programme. Hum Reprod 7, 117-119.

Stones RW, Paterson CM and Saunders NJ (1993) Risk factors for major obstetric haemorrhage. Eur J Obstet Gynecol Reprod Biol 48, 15-18. 
Strandell A, Bergh C and Lundin K (2000) Selection of patients suitable for one-embryo transfer may reduce the rate of multiple births by half without impairment of overall birth rates. Hum Reprod 15, 2520-2525.

Sutcliffe AG, Peters CJ, Bowdin S, Temple K, Reardon W, Wilson L, Clayton-Smith J, Brueton LA Bannister W and Maher ER (2006) Assisted reproductive therapies and imprinting disorders--a preliminary British survey. Hum Reprod 21, 1009-1011.

Sutton ML, Gilchrist RB and Thompson JG (2003) Effects of in-vivo and in-vitro environments on the metabolism of the cumulus-oocyte complex and its influence on oocyte developmental capacity. Hum Reprod Update 9, 35-48.

Tan SL, Royston P, Campbell S, Jacobs HS, Betts J, Mason B and Edwards RG (1992) Cumulative conception and livebirth rates after in-vitro fertilisation. Lancet 339, 1390-1394.

Tatemoto H, Sakurai N and Muto N (2000) Protection of porcine oocytes against apoptotic cell death caused by oxidative stress during In vitro maturation: role of cumulus cells. Biol Reprod 63, 805-810.

146 Taylor A (2003) ABC of subfertility: extent of the problem. BMJ 327, 434-436.

Templeton A, Morris JK and Parslow W (1996) Factors that affect outcome of in-vitro fertilisation treatment. Lancet 348, 1402-1406.

Templeton A and Morris JK (1998) Reducing the risk of multiple births by transfer of two embryos after in vitro fertilization. N Engl J Med 339, 573-577.

Tesarik J, Junca AM, Hazout A, Aubriot FX, Nathan C, Cohen-Bacrie P and Dumont-Hassan M (2000) Embryos with high implantation potential after intracytoplasmic sperm injection can be recognized by a simple, non-invasive examination of pronuclear morphology. Hum Reprod 15, 1396-1399.

Thomas PD, Campbell MJ, Kejariwal A, Mi H, Karlak B, Daverman R, Diemer K, Muruganujan A and Narechania A (2003) PANTHER: a library of protein families and subfamilies indexed by function. Genome Res 13, 2129-2141.

Thomas PD, Kejariwal A, Guo N, Mi H, Campbell MJ, Muruganujan A and Lazareva-Ulitsky B (2006) Applications for protein sequence-function evolution data: mRNA/protein expression analysis and coding SNP scoring tools. Nucleic Acids Res 34, W645-650.

Thurin A, Hausken J, Hillensjo T, Jablonowska B, Pinborg A, Strandell A and Bergh C (2004) Elective single-embryo transfer versus double-embryo transfer in in vitro fertilization. N Engl J Med 351, 2392-2402.

Tiitinen A, Halttunen M, Harkki P, Vuoristo P and Hyden-Granskog C (2001) Elective single embryo transfer: the value of cryopreservation. Hum Reprod 16, 1140-1144.

Tiitinen A, Unkila-Kallio L, Halttunen M and Hyden-Granskog C (2003) Impact of elective single embryo transfer on the twin pregnancy rate. Hum Reprod 18, 1449-1453.

Tong S and Short RV (1998) Dizygotic twinning as a measure of human fertility. Hum Reprod 13, 95-98.

Tsai YC, Chung MT, Sung YH, Tsai TF, Tsai YT and Lin LY (2002) Clinical value of early cleavage embryo. Int J Gynecol Obstet 76, 293-297.

Tummers P, De Sutter P and Dhont M (2003) Risk of spontaneous abortion in singleton and twin pregnancies after IVF/ICSI. Hum Reprod 18, 1720-1723.

Tuppin P, Blondel B and Kaminski M (1993) Trends in multiple deliveries and infertility treatments in France. Br J Obstet Gynaecol 100, 383-385. 
Twisk M, Mastenbroek S, van Wely M, Heineman MJ, Van der Veen F and Repping S (2006) Preimplantation genetic screening for abnormal number of chromosomes (aneuploidies) in in vitro fertilisation or intracytoplasmic sperm injection. Cochrane Database Syst Rev CD005291.

Van Blerkom J, Davis PW and Lee J (1995) ATP content of human oocytes and developmental potential and outcome after in-vitro fertilization and embryo transfer. Hum Reprod $10,415-424$.

Van Blerkom J, Antczak M and Schrader R (1997) The developmental potential of the human oocyte is related to the dissolved oxygen content of follicular fluid: association with vascular endothelial growth factor levels and perifollicular blood flow characteristics. Hum Reprod 12, 1047-1055.

Van den Bergh M, Devreker F, Emiliani S and Englert Y (2001) Glycolytic activity: a possible tool for human blastocyst selection. Reprod Biomed Online 3 (suppl 1), 8.

Van Duivenboden YA, Merkus JM and Verloove-Vanhorick SP (1991) Infertility treatment: implications for perinatology. Eur J Obstet Gynecol Reprod Biol 42, 201-204.

van Hout BA, AI MJ, Gordon GS and Rutten FF (1994) Costs, effects and C/E-ratios alongside a clinical trial. Health Econ 3, 309-319.

Van Montfoort AP, Dumoulin JC, Kester AD and Evers JL (2004) Early cleavage is a valuable addition to existing embryo selection parameters: a study using single embryo transfers. Hum Reprod 19, 2103-2108.

Van Montfoort AP, Dumoulin JC, Land JA, Coonen E, Derhaag JG and Evers JL (2005) Elective single embryo transfer (eSET) policy in the first three IVF/ICSI treatment cycles. Hum Reprod 20, 433-436.

Van Montfoort AP, Fiddelers AA, Janssen JM, Derhaag JG, Dirksen CD, Dunselman GA, Land JA, Geraedts JP, Evers JL and Dumoulin JC (2006) In unselected patients, elective single embryo transfer prevents all multiples, but results in significantly lower pregnancy rates compared with double embryo transfer: a randomized controlled trial. Hum Reprod 21, 338-343.

Van Royen E, Mangelschots K, De Neubourg D, Valkenburg M, Van de Meerssche M, Ryckaert G, Eestermans W and Gerris J (1999) Characterization of a top quality embryo, a step towards single-embryo transfer. Hum Reprod 14, 2345-2349.

Van Royen E, Mangelschots K, Vercruyssen M, De Neubourg D, Valkenburg M, Ryckaert G and Gerris J (2003) Multinucleation in cleavage stage embryos. Hum Reprod 18, 1062-1069.

Vandesompele J, De Preter K, Pattyn F, Poppe B, Van Roy N, De Paepe A and Speleman F (2002) Accurate normalization of real-time quantitative RT-PCR data by geometric averaging of multiple internal control genes. Genome Biol 3, RESEARCH0O34.

Veleva Z, Vilska S, Hyden-Granskog C, Tiitinen A, Tapanainen JS and Martikainen H (2006) Elective single embryo transfer in women aged 36-39 years. Hum Reprod 21, 20982102.

Vilska S, Tiitinen A, Hyden-Granskog C and Hovatta $O$ (1999) Elective transfer of one embryo results in an acceptable pregnancy rate and eliminates the risk of multiple birth. Hum Reprod 14, 2392-2395.

Waterstone J, Parsons J and Bolton V (1991) Elective transfer of two embryos. Lancet 337 , 975-976. 
Wenstrom KD and Gall SA (1988) Incidence, morbidity and mortality, and diagnosis of twin gestations. Clin Perinatol 15, 1-11.

Westergaard T, Wohlfahrt J, Aaby P and Melbye M (1997) Population based study of rates of multiple pregnancies in Denmark, 1980-94. BMJ 314, 775-779.

Wharf E, Dimitrakopoulos A, Khalaf $Y$ and Pickering S (2004) Early embryo development is an indicator of implantation potential. Reprod Biomed Online 8, 212-218.

Winter E, Wang J, Davies MJ and Norman R (2002) Early pregnancy loss following assisted reproductive technology treatment. Hum Reprod 17, 3220-3223.

Wittemer C, Bettahar-Lebugle K, Ohl J, Rongieres C, Nisand I and Gerlinger P (2000) Zygote evaluation: an efficient tool for embryo selection. Hum Reprod 15, 2591-2597.

Wolner-Hanssen P and Rydhstroem H (1998) Cost-effectiveness analysis of in-vitro fertilization: estimated costs per successful pregnancy after transfer of one or two embryos. Hum Reprod 13, 88-94.

Wright VC, Schieve LA, Reynolds MA and Jeng $G$ (2005) Assisted reproductive technology surveillance--United States, 2002. MMWR Surveill Summ 54, 1-24.

Zhang X, Jafari N, Barnes RB, Confino E, Milad M and Kazer RR (2005) Studies of gene expression in human cumulus cells indicate pentraxin 3 as a possible marker for oocyte quality. Fertil Steril 83 Suppl 1, 1169-1179.

Ziebe S, Petersen K, Lindenberg S, Andersen AG, Gabrielsen A and Andersen AN (1997) Embryo morphology or cleavage stage: how to select the best embryos for transfer after in-vitro fertilization. Hum Reprod 12, 1545-1549.

ZonMw (2005) Vruchtbaarheidsstoornissen: Kansen voor doelmatiger zorg (Fertility disorders: Probabilities for efficient care). File number 80-007024-99, June 2005. 


\section{Summary}

A twin pregnancy is, due to its complications, regarded a disadvantageous outcome of an IVF treatment. For the Netherlands an estimated $2.2 \%$ of all newborn children is conceived by IVF. Of these, $32 \%$ is part of a twin. The complications, like hypertension in the mother and preterm delivery and low birth weight of the children, lead to high health care costs, which increase even more when the costs for life-long handicaps and developmental problems are included. The twin pregnancies result from the transfer of two or more embryos per cycle. By transferring only one embryo (single embryo transfer, SET) twin pregnancies can be avoided. The aim of this thesis was to compare several SET transfer strategies from a clinical and cost-effectiveness point of view, and to improve the selection of the single best embryo for SET.

In chapter 2, a transfer policy is described for patients with at least two embryos available. Patients younger than 38 years with at least one embryo of good quality received one embryo (around $20 \%$ of the patients). Patients of 38 years or older or patients with moderate quality embryos received two embryos (DET, $80 \%$ of the patients). In the first as well as the second and third treatment cycle, a statistically similar pregnancy rate was obtained for both groups (32.7 and 33.2\%; 36.1 and $22.9 \%$; and 20.0 and $24.2 \%$, respectively). Because in significantly more SET cycles embryos could be frozen, the cumulative pregnancy rate after the transfer of both fresh and, when appropriate, frozen embryos was significantly higher after SET as compared to DET. However, the overall twin pregnancy rate was still $22.9 \%$.

In a subsequent time-period, the above mentioned transfer strategy was applied in the first cycle with liberalized embryo selection criteria. This resulted in $45 \%$ SETs and 55\% DETs. The pregnancy rates were similar again (33.0 and $30.3 \%$, respectively) and the overall twin pregnancy rate was $12.9 \%$ (chapter 3).

To abolish twin pregnancies, all IVF patients should receive one embryo. In a randomized controlled trial, described in chapter 3 , in which patients with at least two embryos available were randomized for their first cycle between SET or DET irrespective of their age and of the embryo quality, it appeared that the pregnancy rate would decrease significantly if SET was applied in all cycles (pregnancy rate was $21.4 \%$ after SET and $40.3 \%$ after DET). The twin pregnancy rate was reduced from $21.0 \%$ after DET to $0 \%$ after SET. Alongside this clinical trial, a cost-effectiveness analysis on both strategies (SET or DET in all IVF patients, chapter 4) was performed. The costs of an IVF treatment including the costs of a possible pregnancy and delivery (until 4 weeks after delivery) were on average $€ 7334$ for SET 
and $€ 10924$ for DET. This difference was mainly caused by the augmented costs for pregnancy and delivery in the DET strategy. This more expensive strategy however, also led to more successful pregnancies. Each additional successful pregnancy obtained with a DET strategy as compared to the SET strategy cost $€ 19096$ extra.

Finally, in chapter 5 both transfer strategies were combined. All patients younger than 38 years with at least two embryos available received one embryo in their first cycle. If in the second or third treatment cycle one embryo of good quality was available SET and otherwise DET was applied (group A). In the control group (group B), the transfer strategy for the first cycle was similar to that in the second and third cycle; SET when at least one embryo of good quality was available and otherwise DET. The live birth rates after 3 cycles were similar in both groups (62.4 and 62.6\%, respectively). However, the twin pregnancy rate was not reduced by 150 transferring one embryo in the first cycle of all IVF patients (10.1\% group A and $13.4 \%$ group $B$ ). Furthermore, group $A$ required significantly more fresh (2.0 vs 1.8 ) and frozen cycles (0.8 and 0.5 ) to achieve a similar pregnancy rate than group $B$.

To increase the pregnancy rate with SET, more information is needed about the selection criteria for an embryo with a high implantation potential. Besides the established cell number and embryo morphology on the day of transfer, the timing of the first cleavage division seemed important (chapter 6). Embryos that cleaved to the two-cell stage between 23$26 \mathrm{~h}$ post injection (ICSI) or 25-28h post-insemination (IVF), implanted significantly more often as compared to late cleaving embryos. The transfer of a single early cleavage embryo resulted in $37.1 \%$ of the patients in a pregnancy while this was the case in only $10.3 \%$ of the patients receiving a non-early cleavage embryo. A logistic regression analysis revealed that early cleavage was a predictor of pregnancy and blastocyst formation independent of cell number and embryo morphology.

Morphological aspects are insufficient to characterize a good quality embryo. Also from within the embryo or the oocyte important information can be obtained. In chapter 7, a study is described in which the genomewide gene expression in cumulus cells was analysed. These cells surround the intra-follicular oocytes. Differences in intrafollicular conditions important for oocyte and embryo development might be reflected in differences in expression of several genes in cumulus cells. Microarrays were used to analyse genome-wide differential gene expression in cumulus cells from oocytes resulting in good and moderate quality embryos. The expression of 611 genes, among others involved in cell cycle, angiogenesis, apoptosis, growth factor, chemokine and cytokine signaling and hypoxia, was related to embryo quality. 
Although further research is needed, these results might point towards a stress-inducing environment for oocytes that result in moderate quality embryos.

In chapter 8 the results are discussed and the opinion of several actors involved in transfer policy making, like medical practitioners, patients, politicians, embryologists and health economists is described. In conclusion, a SET strategy is at the moment not suitable for all patients, but only for a selected group of top quality patients with top quality embryos. The exact selection criteria, not only embryo selection criteria but also patient selection criteria need to be further developed.v 


\section{Samenvatting}

Eentweelingzwangerschapwordt,vanwege debijbehorendecomplicaties, gezien als een nadeel van een IVF behandeling. In Nederland is naar schatting $2,2 \%$ van de pasgeboren kinderen het resultaat van IVF. Hiervan behoort $32 \%$ tot een tweeling. De complicaties, zoals hypertensie bij de moeder en vroeggeboorte en verlaagd geboortegewicht bij de kinderen leiden tot hoge kosten in de gezondheidszorg. Deze kosten worden nog hoger als ook de kosten van levenslange ontwikkelingsproblemen en chronische ziektes ten gevolge van de tweelingzwangerschappen meegenomen worden. De tweelingzwangerschappen ontstaan doordat vaak meer embryo's tegelijk terug geplaatst worden. Door maar één embryo terug te plaatsen (=single embryo transfer, SET) zouden tweelingzwangerschappen voorkomen kunnen worden. Het doel van dit

152 proefschrift was dan ook het analyseren van verschillende SET strategieën vanuit een klinisch en kosten-effectief oogpunt en het verbeteren van de selectie van het embryo met de hoogste implantatie kans.

In hoofdstuk 2 wordt een terugplaatsstrategie beschreven voor patiënten met tenminste 2 embryo's. Patiënten jonger dan 38 jaar met tenminste 1 embryo van goede kwaliteit kregen één embryo terug (ongeveer $20 \%$ van de patiënten). Bij patiënten van 38 jaar en ouder en patiënten met minder goede embryo's werden twee embryo's teruggeplaatst (DET, $80 \%$ van de patiënten). Zowel in de $1^{e}$ als in de $2^{e}$ en $3^{e}$ behandelcyclus was de zwangerschapskans statistisch vergelijkbaar voor SET en DET (respectievelijk 32,7 en $33,2 \%, 36,1$ en $22,9 \%$, en 20,0 en $24,2 \%$ ). Omdat na SET significant vaker embryo's konden worden ingevroren, was het cumulatieve zwangerschapspercentage na de terugplaatsing van zowel verse als ingevroren embryo's na SET significant hoger dan na DET. Met deze terugplaatsstrategie was het tweelingzwangerschapspercentage van alle IVF zwangerschappen echter nog steeds hoog, namelijk $23 \%$.

In een volgend cohort werd bovenstaande terugplaatsstrategie herhaald voor de $1^{e}$ cyclus, waarbij de criteria voor een kwalitatief goed embryo werden versoepeld. Hierdoor kregen $45 \%$ van de patiënten één embryo terug en $55 \%$ twee. De zwangerschapskansen waren wederom vergelijkbaar voor SET en DET (respectievelijk 33,0 en 30,3\%) en het tweelingzwangerschapspercentage was $12,9 \%$ voor de hele IVF groep (hoofdstuk 3).

Om het tweelingzwangerschapspercentage zo laag mogelijk te krijgen zou bij àlle IVF patiënten één embryo teruggeplaatst moeten worden. Uit een gerandomiseerde studie, beschreven in hoofdstuk 3, waarin alle patiënten met tenminste 2 embryo's in hun eerste cyclus werden gerandomiseerd tussen SET of DET, ongeacht leeftijd en ongeacht embryo kwaliteit, bleek echter dat de kans op zwangerschap significant zou dalen als bij àlle IVF patiënten één embryo teruggeplaatst zou worden 
(zwangerschapskans was $21,4 \%$ na SET en $40,3 \%$ na DET). Het percentage tweelingzwangerschappen werd teruggebracht van $21,0 \%$ na DET naar $0 \%$ na SET. Parallel aan deze klinische studie werd een kosteneffectiviteits analyse gedaan van beide strategieën (SET of DET in alle patiënten, hoofdstuk 4). De kosten van een IVF behandeling inclusief de kosten van een eventuele zwangerschap tot 4 weken na de bevalling waren gemiddeld $€ 7334$ voor de SET strategie en $€ 10924$ voor de DET strategie. Dit verschil werd voornamelijk veroorzaakt door de hogere kosten tijdens zwangerschap en bevalling na DET. Deze duurdere strategie leidde echter ook tot meer succesvolle zwangerschappen. Elk levend geboren kind dat extra geboren werd na DET ten opzichte van eSET kostte $€ 19096$.

Als laatste werden in hoofdstuk 5 beide terugplaatsstrategieën gecombineerd. Patiënten jonger dan 38 jaar met minimaal 2 embryo's kregen allemaal SET in de eerste cyclus. Als er in de $2^{e}$ of $3^{e}$ cyclus een embryo van goede kwaliteit aanwezig was, kregen ze ook dan SET. Waren in de $2^{e}$ of $3^{e}$ cyclus alleen embryo's van mindere kwaliteit beschikbaar, dan werden er twee embryo's teruggeplaatst (groep A). Voor de controle groep (groep B, ook patiënten jonger dan 38 jaar met tenminste 2 embryo's beschikbaar) was de $1^{e}$ cyclus gelijk aan cyclus 2 en 3; alleen SET als er een embryo van goede kwaliteit aanwezig was, anders DET. De cumulatieve kans op een levend geboren kind na 3 cycli was in beide groepen even hoog (respectievelijk 62,4 en 62,6\%). Echter het percentage tweelingzwangerschappen werd niet verlaagd door bij iedereen in de $1^{\mathrm{e}}$ cyclus één embryo terug te plaatsen (10,1\% voor groep A en 13,4\% voor groep B) en bovendien moest deze groep meer puncties (respectievelijk 2.0 en 1.8) en meer vries-dooicycli (respectievelijk 0.8 en 0.5 ) ondergaan om tot hetzelfde zwangerschapspercentage te komen.

Om de zwangerschapskans met SET te verhogen is meer informatie nodig over de criteria waaraan een embryo met een hoge implantatie kans moet voldoen. Naast de veel gebruikte criteria celaantal en embryomorfologie op de dag van terugplaatsing bleek het tijdstip van de eerste klievingsdeling een belangrijke factor (hoofdstuk 6). Embryo's die 23-26 uur na injectie (ICSI) of 25-28 uur na inseminatie (IVF) reeds uit twee cellen bestonden, implanteerden significant vaker dan embryo's die pas later kliefden. Het terugplaatsen van één vroeg gekliefd embryo resulteerde in een doorgaand zwangerschapspercentage van 37,1\%, terwijl het terugplaatsen van één niet-vroeg gekliefd embryo maar in $10,3 \%$ van de patiënten in een zwangerschap resulteerde.

De vraag was echter of vroege klieving een onafhankelijke voorspeller voor zwangerschap was, of dat vroege klieving gerelateerd was aan celaantal en embryomorfologie op dag 2 en daardoor geen extra informatie kon verschaffen. Met behulp van een logistische regressie-analyse bleek dat 
vroege klieving, naast celaantal en embryomorfologie, een onafhankelijke voorspeller was voor zowel zwangerschap als blastocystvorming.

Niet alleen uiterlijke kenmerken karakteriseren de kwaliteit van een embryo. Ook binnenin een eicel of embryo bevindt zich informatie die gerelateerd kan worden aan embryokwaliteit. In hoofdstuk 7 staat een onderzoek beschreven waarbij is gekeken naar de gen-expressie in cumuluscellen. Deze cellen omgeven de intra-folliculaire eicellen. Verschillen in intrafolliculaire condities die voor de eicel en latere embryo ontwikkeling van belang zijn, worden dus mogelijk weerspiegeld in verschillen in expressie van bepaalde genen in de cumuluscellen. Om deze verschillen op te sporen werd met behulp van microarrays naar de genoom-brede genexpressie gekeken in cumuluscellen waarvan de eicellen uitgroeiden tot kwalitatief goede en minder goede embryo's. De expressie van

154611 genen bleek gerelateerd te zijn aan embryo-ontwikkeling. Deze genen waren onder andere betrokken bij de celcyclus, angiogenese, apoptose, groeifactorproductie en chemokine- en cytokinesignalering en hypoxie. Ook al is verder onderzoek nodig, deze resultaten duiden mogelijk op intra-folliculaire stress omstandigheden ten gevolge waarvan de eicellen uit zouden kunnen groeien tot embryo's met een verlaagde implantatiekans.

In hoofdstuk 8 worden de resultaten bediscussieerd en wordt de mening van verschillende actoren die betrokken zijn bij het opstellen van een terugplaatsbeleid bij IVF, zoals clinici, patiënten, embryologen, politici en gezondheidseconomen, besproken. Concluderend blijkt dat op dit moment een SET strategie niet voor alle IVF patiënten geschikt is, maar dat het alleen toegepast dient te worden in een geselecteerde groep. Wat die selectie criteria zijn, niet alleen de embryo selectie criteria maar ook de patiënt-selectiecriteria, dient nog verder onderzocht te worden. 


\section{Dankwoord}

En dan bijna helemaal achteraan, terwijl het door de meesten toch als eerste gelezen wordt, het dankwoord. Want ook al staat mijn naam alleen op de voorkant van dit proefschrift, er zijn vele mensen geweest zonder wie dit boekje er nooit was geweest. Behalve alle IVF patiënten die op een belangrijk punt in hun leven bereid waren mee te doen aan een onderzoek, zijn er ook een aantal mensen die ik met naam wil bedanken.

Allereerst mijn promotoren Prof. Evers en Prof. Geraedts. In de beginjaren hebben jullie me vooral van een afstandje in de gaten gehouden. Maar toen het echt nodig was hebben jullie mij zeer gericht door de 'terminale fase' heen geholpen. Jullie inmenging, goede ideeën, snelle controles, interesse 'hoe is het met de wetenschap' en de RBM delivery service hebben zeker een positieve invloed op dit proefschrift gehad.

Daarnaast hoort natuurlijk John Dumoulin. John, dankzij jou was er een leuk en interessant project en dankzij jou mocht ik aan dat project werken. Ook al zaten we misschien niet altijd op één lijn (het plan van aanpak jeukt waarschijhlijk nog steeds), toch heb ik ontzettend veel aan jou gehad. Zo begrijp ik na al die jaren eindelijk een beetje hoe het uitstervende dBase werkt, kan ik de enige echte 'wetensjappelijke' Dumoulin tabellen interpreteren en weet ik zelfs de waarde van een simpel klein spijkertje voor een heel huis te waarderen. Je zeer beschrijvende verhalen over jouw ervaringen samen met je nooit aflatende opgewektheid en positivisme hebben me meermalen met een lach de problemen en tegenslagen doen relativeren, 'zeg maar'.

Marij. Wat ben ik ontzettend blij geweest met jouw ondersteuning van het onderzoek. Patiënten voorlichten, gegevens invoeren, bellen en vele brieven versturen. Je hebt me echt werk uit handen genomen, waar ik je heel dankbaar voor ben.

Marijke en Marion. Ik ben erg verheugd dat jullie m'n paranimfen willen zijn! Jullie hebben me vaak letterlijk tot grote adembenemende hoogten gebracht. Zonder jullie had ik dan ook nog nooit een ijsbeer gezien, was ik nooit van een berg naar onder geroetsjt en had ik nooit tot stilstand mogen komen op een terras (mogen die foto's alsnog gedeleted worden?), hadden we nooit op 1 januari al de slingers en ballonnen voor carnaval gehad (inclusief bijbehorende muziek, fijn he mensen), was het heel stil geweest op de dansvloer van menig feestje, en was het na 17u wel heel uitgestorven geweest op het lab. 
Edith en Josien. Als 'piep' heb ik veel aan jullie onderzoeks- en promotieervaringen gehad. Jullie oprechte interesse, meedenken, ideeën, adviezen en medewerking hebben een belangrijke bijdrage aan dit proefschrift geleverd.

Mijn lieve lab collega's Inge, Marij, Marijke, Marion, Myriam en Roel. De vele verschillende persoonlijkheden brachten altijd veel gezelligheid op het kantoor/koffiekamer. Ik ben dan ook nog altijd heel blij met m'n werkplek in de 'koffiekamer', ook om nog een beetje feeling met jullie werkzaamheden te kunnen houden. Zonder jullie inzet was het SET/DET onderzoek en het verzamelen van de cumulus cellen niet zo soepel en probleemloos als nu verlopen. Ik heb echt bewondering gekregen voor hoe jullie met de embryo's omgaan en er alles voor over hebben om ze een zo goed mogelijk leven te geven. Met zo'n pleegouders moeten de embryo's het heerlijk hebben bij ons.

Jolande en Dr. D. Dank voor alle hulp bij het opzetten van het onderzoek, tijdens de dip in IVF patienten en bij het opschrijven van de resultaten.

De buren, Annemieke, Carla, Cecile, Germaine, Janneke, Kim, Laurence en Marie-Jose: Als er iemand blij is dat de SET studie afgelopen is, dan zijn julie het wel. Ruimte gebrek, statussen zoek, patiënten weg, dat jullie me niet schreeuwend de afdeling afgezet hebben is me nog steeds een raadsel. Dank voor jullie geduld.

Audrey, Carmen en Prof Severens. Hartelijk dank voor de prettige en 'doelmatige' samenwerking. KEA, ICER, DBC, er is een kosten-effectieve wereld voor me open gegaan. Door jullie betrokkenheid hebben we SET en DET vanuit meerdere perspectieven kunnen bekijken waardoor het naar mijn idee een heel interessant project is geworden. Audrey, veel succes met de laatste loodjes!

Torik, Fons en Bieke: Nog zo'n nieuwe wereld. Hoe kun je iets zinnigs zeggen over 16x55.000 gegevenspunten? Ook al ben ik door alle genen het bos wel eens kwijt geraakt, toch ben ik erg blij Bieke, dat jij die gegevenspunten hebt willen genereren. Maar, ik ben ook wel erg blij dat Fons en Torik het tot voor mij behapbare en begrijpbare brokken hebben kunnen reduceren en dat jullie me met zo veel geduld en enthousiasme geholpen hebben met het realiseren van hoofdstuk 7 .

Arnold Kester: Het is alweer een tijdje geleden, maar het enthousiasme voor de logistische regressie ben ik nog niet vergeten. 
Natuurlijk zijn er ook veel mensen geweest die buiten het werk hun steun aan dit proefschrift hebben gegeven. Ik wil iedereen dan ook hartelijk danken voor alle interesse in m'n werk van de afgelopen jaren. Met name de gezellige, ongedwongen, mede-(ex)-promovendi dames Munnix (Frusti 1 of 2?), Kim en Bosch (waar begin je aan?) èn Suzanne (ech niet) en Koen (ech wel) wil ik bedanken voor de goede vriendschap, zeer gezellige en ongedwongen weekendjes, analyses van de Corleone familie, etentjes en de niet altijd zo ontspannen wandelingen. Ze hebben de gedachten meermalen verzet en hopelijk houden we dit er ook na alle promoties nog in.

Frank en Inge. Dank jullie wel voor alle goede gesprekken, adviezen (niet alleen op promotie gebied) en de bijvoeding. Ik vind het ontzettend jammer dat jullie gaan verhuizen en zal jullie zeker missen. Gelukkig is Brabant dicht bij en ken't 'r ok kei gezellig zien.

Bregje, kleine grote zus: Ook al leef je in je eigen wereld en ook al heb je er geen idee van wat er in mijn leven allemaal gebeurd, toch wil ik ook jou bedanken voor de steun die je me op jouw manier hebt gegeven. Hoe gelukkig jij bent en hoe tevreden met zo'n kleine dingen, daar kunnen veel mensen nog een voorbeeld aan nemen. Ik ben echt supertrots op je!

En tenslotte natuurlijk pap en mam. Volgens mij staat er in dit boekje voor jullie niets nieuws meer. Jullie hadden zoveel oprechte interesse voor $\mathrm{m}$ 'n werk, af en toe zelfs misschien een beetje te veel. En altijd stonden jullie klaar om naar m'n klaagzang te luisteren als het in mijn ogen weer eens tegen zat. Ook hoe trots jullie op mij zijn mag iedereen (helaas) weten. Toch geeft het wel aan hoeveel vertrouwen jullie in mij hebben. Bedankt pap en mam, voor alles, ik hou van jullie en een dikke kus! 


\section{Curriculum Vitae}

Aafke van Montfoort werd op 2 oktober 1979 geboren te Weert. In 1997 behaalde zij haar VWO-diploma aan de Scholengemeenschap Bisschoppelijk College te Weert. In datzelfde jaar begon zij aan de Universiteit Maastricht met de studie Gezondheidswetenschappen, met als afstudeerrichting Biologische Gezondheidkunde. Tijdens haar afstudeerstage bij de afdeling Medische Microbiologie van het Academisch ziekenhuis Maastricht deed zij, onder leiding van dr. C. Vink en dr. J. Heemskerk, onderzoek naar de betrokkenheid van R33en R78-eiwit bij calcium signalering in rat cytomegalovirus. Aansluitend deed zij een tweede stage bij de afdeling fysiologie aan de Universiteit van Cambridge (Engeland), onder leiding van Dr. S. Sage en Dr. J. Heemskerk. Hier onderzocht zij de regulatie van 'store mediated calcium entry' door glycoprotein VI en collageenreceptoren in bloedplaatjes. Eind 2001 behaalde zij haar doctoraal diploma, waarna zij vanaf begin 2002, als promovendus binnen het IVF laboratorium van het Academisch ziekenhuis Maastricht, de in dit proefschrift beschreven onderzoeken heeft gedaan. Het onderzoek werd uitgevoerd binnen de divisie Ontwikkelingsbiologie van het onderzoeksinstituut Groei en Ontwikkeling (GROW) en stond onder leiding van Dr. J.C.M. Dumoulin Prof. J.L.H. Evers en Prof. J.P.M. Geraedts. Sinds april 2007 voert zij binnen datzelfde IVF laboratorium onderzoek uit naar de invloed van in vitro fertilisatie (IVF) technieken op de X-inactivatie en imprinting-status van uit IVF voortkomende embryo's.

\section{Publicaties}

Van Montfoort A.P.A., Fiddelers A.A.A., Land J.A., Dirksen C.D., Severens J.L., Geraedts J.P.M., Evers J.L.H., John C.M. Dumoulin (2007) eSET irrespective of the availability of a good quality embryo in the first cycle only is not effective in reducing overall twin pregnancy rates. Hum Reprod, in press.

Fiddelers A.A.A., Van Montfoort A.P.A., Dirksen, C.D., Dumoulin, J.C.M., Land, J.A., Dunselman, G.A.J., Janssen, J.M., Severers, J.L., Evers, J.L.H. (2006) Single versus double embryo transfer: cost-effectiveness analysis alongside a randomized clinical trial. Hum Reprod 21, 2090-2097.

Van Montfoort, A.P.A., Fiddelers, A.A.A., Janssen, J.M., Derhaag, J.G., Dirksen, C.D., Dunselman, G.A.J., Land, J.A., Geraedts, J.P.M., Evers, J.L.H., Dumoulin, J.C.M. (2006) In unselected patients, elective single embryo transfer prevents all multiples, but causes significantly lower pregnancy rates as compared to double embryo tranfser; a randomized controlled trial. Hum Reprod 21, 338343. 
Dumoulin, J.C.M, Derhaag, J.G., Bras, M., Van Montfoort, A.P.A., Kester, A.D.M., Evers, J.L.H., Geraedts, J.P.M., Coonen, E. (2005) Growth rate of human preimplantation embryos is sex dependent after ICSI but not after IVF. Hum Reprod, 20, $484-491$.

Van Montfoort, A.P.A, Dumoulin, J.C.M., Land, J.A., Coonen, E., Derhaag, J.G. and Evers, J.L.H. (2005) Elective single embryo transfer (eSET) policy in the first three IVF/ICSI treatment cycles. Hum Reprod, 20, 433-436.

Van Montfoort, A.P.A, Dumoulin, J.C.M., Kester, A.D.M. and Evers, J.L.H. (2004) Early cleavage is a valuable addition to existing embryo selection parameters: a study using single embryo transfers. Hum Reprod, 19, 2103-2108.

Abstracts

Van Montfoort, A.P.A., Fiddelers, A.A.A., Janssen, J.M., Derhaag, J.G., Dirksen, C.D., Dunselman, G.A.J., Land, J.A., Geraedts, J.P.M., Evers, J.L.H., Dumoulin, J.C.M. (2006) SET in the first treatment cycle of all IVF patients? Hum Reprod, 21, suppl $1, \mathrm{i} 147$.

Fiddelers A.A.A., Dirksen C.D., Van Montfoort A.P.A., Dumoulin J.C.M., Evers J.L.H., Severens J.L. (2005) Economic evaluation of single and double embryo transfer: results of a Markov model. Hum Reprod, 20, suppl 1, i142.

Van Montfoort, A.P.A., Fiddelers, A.A.A., Janssen, J.M., Derhaag, J.G., Dirksen, C.D., Dunselman, G.A.J., Land, J.A., Geraedts, J.P.M., Evers, J.L.H., Dumoulin, J.C.M. (2005) Single embryo transer (SET) in unselected patients: a randomized controlled trial (RCT). Hum Reprod, 20 suppl 1, 55.

Van Montfoort, A.P.A., Janssen J.M., Fiddelers, A.A.A., Derhaag, J.G., Dirksen, C.D., Evers, J.L.H. and Dumoulin, J.C.M. (2004) Single versus double embryo transfer: a randomised study. Hum Reprod, 19 suppl 1, 134.

Van Montfoort, A.P.A, Kester, A.D.M., Evers, J.L.H. and Dumoulin, J.C.M. (2003) Early embryo cleavage as a selection criterion improves single embryo transfer results. Hum Reprod, 18 suppl 1, 20.

Dumoulin JCM, Van Montfoort APA, Land JA, Derhaag JG, Coonen E, Evers JLH (2003) Results of elective single embryo transfer (eSET) in the first three treatment cycles. Hum Reprod, 18 suppl 1, xviii 132. 